University of Louisville ThinkIR: The University of Louisville's Institutional Repository

Electronic Theses and Dissertations

$8-2019$

\title{
Predictors of empirically-supported interventions following structured functional assessment.
}

Alton Nathaniel Verbist

University of Louisville

Follow this and additional works at: https://ir.library.louisville.edu/etd

Part of the Social Work Commons

\section{Recommended Citation}

Verbist, Alton Nathaniel, "Predictors of empirically-supported interventions following structured functional assessment." (2019). Electronic Theses and Dissertations. Paper 3289.

https://doi.org/10.18297/etd/3289

This Doctoral Dissertation is brought to you for free and open access by ThinkIR: The University of Louisville's Institutional Repository. It has been accepted for inclusion in Electronic Theses and Dissertations by an authorized administrator of ThinkIR: The University of Louisville's Institutional Repository. This title appears here courtesy of the author, who has retained all other copyrights. For more information, please contact thinkir@louisville.edu. 


\title{
PREDICTORS OF EMPIRICALLY-SUPPORTED INTERVENTIONS FOLLOWING STRUCTURED FUNCTIONAL ASSESSMENT
}

\author{
By \\ Alton Nathaniel Verbist \\ B.A., Union University, 2003 \\ M.S.S.W., University of Louisville, 2014

\begin{abstract}
A Dissertation
Submitted to the Faculty of the

Raymond A. Kent School of Social Work of the University of Louisville in Partial Fulfillment of the Requirements

for the Degree of

Doctor of Philosophy

in Social Work
\end{abstract}

Raymond A. Kent School of Social Work

University of Louisville

Louisville, Kentucky

And

College of Social Work

University of Kentucky

Lexington, $\mathrm{KY}$

August 2019 



\title{
PREDICTORS OF EMPIRICALLY-SUPPORTED INTERVENTIONS FOLLOWING STRUCTURED FUNCTIONAL ASSESSMENT
}

\author{
By \\ Alton Nathaniel Verbist \\ B.A., Union University, 2003 \\ M.S.S.W., University of Louisville, 2014
}

A Dissertation Approved on

June 14, 2019

by the following Dissertation Committee:

Dr. Crystal Collins-Camargo

Dr. Becky Antle

Dr. Emma Sterrett-Hong

Dr. Heehyul Moon

Dr. Christopher Flaherty 


\section{ACKNOWLEDGEMENTS}

I extend my deepest gratitude to my wife, Bobbie, for her patience and sacrifice during this long process. I would like to thank Dr. Crystal Collins-Camargo for her continual support, guidance, and encouragement. I would also like to thank all of my other committee members for their insight and assistance. 


\section{ABSTRACT \\ PREDICTORS OF EMPIRICALLY-SUPPORTED INTERVENTIONS FOLLOWING STRUCTURED FUNCTIONAL ASSESSMENT}

Alton Nathanial Verbist

June 14, 2019

Assessment is foundational to the process of evidence-based practice yet has received little research attention. Project SAFESPACE has recently initiated screening and assessment for all children entering out of home care in Kentucky with the goal of providing assessment-driven, evidence-based treatment. The Child and Adolescent Needs and Strengths (CANS) assessment — the instrument adopted in this service system-is designed to link the assessment process with the selection of appropriate interventions. Informed by naturalistic decision making, this dissertation sought to investigate the relationship between responses on the CANS assessment and the empirically supported interventions subsequently prescribed by the assessing clinician.

Using secondary data maintained in the statewide automated child welfare information system, a number of descriptive analyses were conducted in order to better understand this redesigned service system including the frequency of specific treatment prescriptions at the case, clinician, and agency levels as well as population-level CANS findings. Building upon these initial inquiries, four multilevel logistic models were developed to examine the relationship between assessment-derived predictors and the 
prescribed treatment modality along four dimensions: trauma exposure, emotional/behavioral problems, family functioning, and substance use.

Descriptive findings reveal an out of home care population with prevalent traumarelated symptoms and extensive emotional/behavioral needs. Considerable variation was observed in the treatment prescription patterns of assessing clinicians. Likewise, there is variation in the degree to which assessment findings align with prescribed treatment modalities. Findings suggest that assessment-derived information may be an important consideration in the prescription of a trauma-focused treatment and a minor, but still possibly salient, factor in treatment decision making related to emotional/behavioral needs and substance use. There appears to be no meaningful relationship between assessment responses and recommendations for family-focused treatment.

As interpreted through the lens of naturalistic decision making, the results suggest that while assessment responses may one source of information utilized by clinicians in the field when making treatment decisions, there is likely a differential degree of influence depending on the specific dimension of treatment. Findings provide a foundation for future research into the situation-action matching decision rules employed by clinicians within this service system, particularly those with established expertise. 


\section{TABLE OF CONTENTS}

ACKNOWLEDGMENTS......................................................................... ii

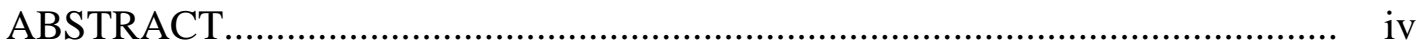

LIST OF TABLES .............................................................................. viii

CHAPTER 1: INTRODUCTION .............................................................. 1

Evidence-Based Practice..................................................................... 1

The Role of Assessment in the EBP Process............................... 3

Assessment in the Child Welfare Context....................... 5

Assessment in Project SAFESPACE.............................. 7

Theoretical Framework......................................................................... 8

Naturalistic Decision Making..................................................... 11

Application of NDM to the Present Study..................... 13

Importance of the Present Study..................................................... 15

CHAPTER 2: REVIEW OF THE LITERATURE........................................ 18

Conceptually-Relevant Literature: Treatment Decision Making.............. 18

Studies Using a Survey or Questionnaire Design....................... 19

Studies Using a Vignette or Case Study Design........................ 23

Studies in a Naturalistic Setting............................................... 31

Summary of the Conceptually-Relevant Literature...................... 33

Concretely-Relevant Literature: Assessment \& ESIs........................... 34

Structure Assessments.............................................................. 35

The CANS Functional Assessment as Predictor......................... 38

Predicting the Use of ESIs........................................................ 44

Summary of the Concretely-Relevant Literature......................... 51

CHAPTER 3: RESEARCH METHODS................................................... 53

Research Context and Design........................................................... 53

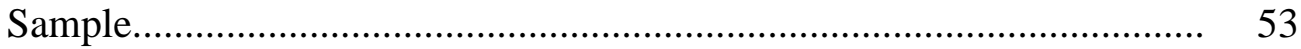

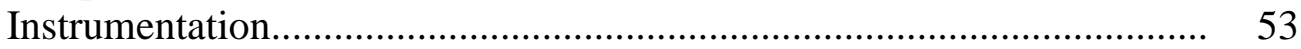

Child and Adolescent Needs and Strengths Assessment............. 54

CANS Assessment Report.................................................. 56

Research Variables....................................................................... 57

Dependent Variables: ESI Selection.......................................... 57

Predictor Variables: Assessment-Identified Needs....................... 61

Analytic Strategy...................................................................... 62

Multilevel Modeling......................................................... 63

Sample Size.......................................................... 67

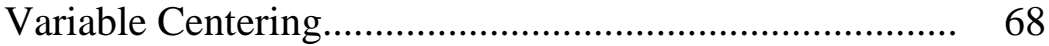

Model Estimation.......................................................... 69 
Model Comparison and Evaluation................................... 69

Conceptual Model.

Model Building Process................................................. 72

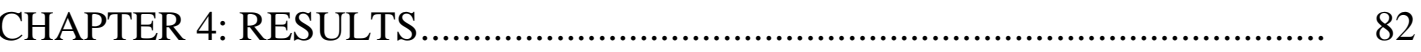

Sample Description.......................................................................... 82

Describing the Service Landscape.......................................................... 84

Prescribed Treatment Modalities................................................................ 84

Focus-of-Treatment Composite Variables..................................... 91

Descriptive Findings from the Initial CANS............................................ $\quad 93$

CANS Modules..................................................................... 93

Trauma Experiences................................................................... 94

Emotional/Behavioral Problems................................................ 96

Family Functioning................................................................. 98

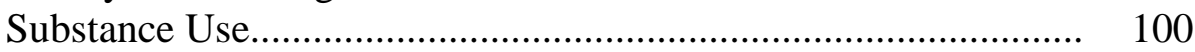

Bivariate Correlation Among Composite Predictor Variables................... 102

Normality of Composite Predictor Variable Scores................................... 102

Child-Level Group Differences in Predictor Variable Scores................... 103

Differences in CANS Domain Scores by Prescribed ESI......................... 105

Multilevel Logistic Regression Modeling................................................ 106

The Unconditional Model and the Intraclass Correlation.............. 106

The Fixed Effects CANS Predictors Only Model......................... 107

The Random Effects CANS Predictors Only Model..................... 108

The Fixed Effects CANS and Demographics Predictors Model... 109

Model Evaluation and Comparison........................................................ 110

Model Interpretation.......................................................................... 112

CHAPTER 5: DISCUSSION, LIMITATIONS, AND CONCLUSIONS.............. 115

Discussion.................................................................................... 115

Treatment Prescription.......................................................... 116

CANS Functional Assessment................................................... 121

Trauma Exposure and Trauma-Related Symptoms....................... 123

Emotional/Behavioral Needs..................................................... 129

Family Functioning.................................................................... 133

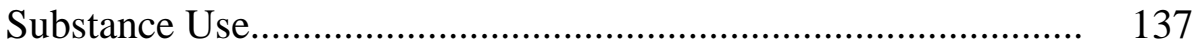

Limitations.......................................................................... 144

Theoretical Integration and Conclusions................................................. 147

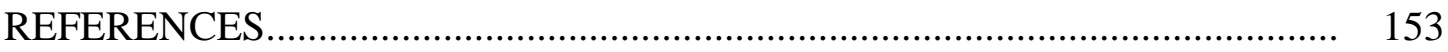

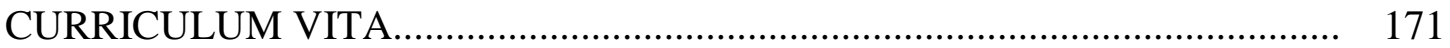




\section{LIST OF TABLES}

TABLE

PAGE

3.1 ESIs and Focus-of-Treatment Composite Variable Inclusion.................... 60

3.2 Predictor Variables \& Associated CANS Domain Items............................ 61

3.3 Model Data Components....................................................................... 65

4.1 Sample Demographics.................................................................. 83

4.2 Frequency of Prescribed Treatment Modalities..................................... 87

4.3 CANS, Clinicians, and Prescribed Modalities by Agency .......................... 88

4.4 Most Homogenous and Eclectic Modality Prescriptions by Clinician........ 91

4.5 "Other" ESIs and Focus-of-Treatment Composite Variable Inclusion......... 92

4.6 Mean Initial CANS Assessments Scores by Domain................................ 93

4.7 Frequency of Triggered Modules Among Initial CANS Assessments........ 94

4.8 Trauma-Related CANS Items............................................................ 96

4.9 Emotional/Behavioral Problems-Related CANS Items............................. 98

4.10 Family Functioning-Related CANS Items............................................. 100

4.11 Substance Use-Related CANS Items................................................ 101

4.12 Correlation Matrix of CANS-Derived Predictor Variables......................... 102

4.13 Group Differences in CANS-Derived Predictor Variable Scores................ 104

4.14 Differences in Scores by Cases Prescribed/Not Prescribed Modalities....... 105

4.15 Unconditional Model (Model 0) Coefficients............................................ 106

4.16 Fixed CANS Predictors Only (Model 1) Coefficients................................ 107

4.17 Random Effects CANS Predictors Only (Model 2) Variance Estimates..... 108

4.18 Fixed CANS and Demographic Predictors Model (Model 3) Coefficients.. 109

4.19 Evaluation and Comparison of Models................................................... 110

4.20 Standardized Regression Coefficients - Model 1 and Model 3.................. 112

4.21 Odds Ratios - Model 3 .......................................................................... 113 


\section{CHAPTER 1:}

\section{INTRODUCTION}

\section{Evidence-Based Practice}

Evidence-based practice (EBP) is "a process for making practice decisions in which practitioners integrate the best research evidence available with their professional expertise and with client attributes, values, preference and circumstances" (Rubin, 2008, p. 7). EBP has been described as "the most challenging and critical practice area of health care and human services" (Roberts \& Yeager, 2004, p. v). Within the field of social work, the emphasis on EBP has steadily gained momentum over the past two decades (Tuten, Morris-Compton, Abrefa-Gyan, Hwang, \& Harrington, 2016).

Many practitioners and academics alike are unclear about what, precisely, evidence-based practice entails (Drisko \& Grady, 2015). As Shlonsky \& Gibbs (2004) have observed, there is danger that the term evidence-based practice will become little more than "a catchphrase for anything that is done with clients that can somehow be linked to an empirical study" (p. 137). As used in the present work EBP refers to an approach to practice intended to assist practitioners in making informed decisions about treatment; as Thyer and Myers (2011) note, "EBP is a process, a verb, not a noun" (p. 8).

The EBP process consists of a series of logically connected steps. While there is some variation in the precise delineation of these steps among different scholars (Grady \& Drisko, 2014), the fundamental EBP process can be conceptualized as follows: (1) convert information needs into an answerable question, (2) locate the best evidence with 
which to answer the question, (3) critically appraise the located evidence, (4) apply the results of the appraisal to policy and practice decisions, and (5) evaluate outcomes (Gibbs \& Gambrill, 2002; Sackett, Richardson, Rosenberg, \& Haynes, 1997; Thyer, 2004).

Despite its emphasis on locating and appraising the best available research, EBP is not driven solely by empirical evidence. Rather, Gilgun (2005) has identified four cornerstones underlying EBP in social work: (1) research and theory, (2) practice wisdom, (3) the person of the practitioner (i.e., assumptions, values, biases, and worldviews), and (4) what the client brings to the practice situation. The first three of these cornerstones are brought into the practice environment independent from the client; as such, practitioners must be prepared to integrate information communicated from the client and modify their clinical responses accordingly (Gilgun, 2005).

Whereas EBP is a process, empirically supported interventions (ESIs) or empirically supported treatments (ESTs) are products—-specifically, therapeutic interventions that have been scientifically validated (Drisko \& Grady, 2015; McBeath, Briggs, \& Aisenberg, 2010; Thyer \& Pignotti, 2011). The two terms (EBP \& ESIs) are conceptually distinct (Thyer \& Pignotti, 2011) though there is still much confusion regarding their respective definitions (Rubin \& Parrish, 2007) and they are often used interchangeably in the scholarly literature (Tuten et al., 2016). The search process inherent to EBP may in fact culminate in the identification of an appropriate ESI (when one exists) but merely selecting a treatment from a list of ESIs is not sufficiently indicative of, or even necessarily consistent with, EBP (Mullen, Bledsoe, \& Bellamy 2008). Rather, the evidence-based practitioner must appraise the scientific evidence underlying particular ESIs, integrate this evidence with other sources of information (e.g. 
practice wisdom), and determine the most appropriate course of action (Thyer \& Pignotti, 2011).

\section{The Role of Assessment in the EBP Process}

The first step in the process of EBP requires one to identify particular information needs such that they may inform the formulation of an answerable question (Gibbs \& Gambrill, 2002). In a practice context, these information needs are recognized through the process of assessment. Simmons (2011) has described thorough assessment as the “necessary first step in the process of EBP” (p. 265). Likewise, Grady and Drisko (2014) describe assessment as the very "foundation" of the entire EBP process (p. 5). A practitioner completing an assessment is engaging in the same general process as the scientific researcher-namely, the systematic gathering of data to answer a question (Sexton \& Kelley, 2010). Indeed, even the act of conducting an assessment provides the clinician with the necessary information from which to develop an answerable question, locate the best research evidence, and appraise the evidence's relevance to the particular client or case.

Standardized assessments in particular can serve as an effective starting point for the evidence-based practice process (Grady \& Drisko, 2014). Research suggests that structured assessments are associated with greater diagnostic accuracy than unstructured approaches (Ponniah et al., 2011). Likewise, research indicates that standardized assessments are perceived as being of a higher quality and more useful for treatment planning compared to unstructured assessment techniques (Andershed \& Andershed, 2016). As such, the importance of an accurate, thorough, and structured assessment can 
hardly be overstated. With a more accurate understanding of the client's unique needs, diagnoses, and circumstances, the clinician is better informed to engage in informed treatment decision making in a manner consistent with the principles of EBP.

Certainly, evidence collected during structured assessment is not the only consideration that influences the selection of a treatment approach. Research indicates that practitioners are influenced by a wide range of factors when deciding on a clinical course of action including ease of implementation, flexibility, peer recommendation, accessibility to training and supervision, approval for insurance reimbursement, length of treatment, and personal experience (Nelson, Steele, \& Mize, 2006; Nelson \& Steele, 2008). EBP models recognize that practitioners' decisions are informed by variables beyond empirical evidence such as that collected during a structured assessment (Haynes, Devereaux, \& Guyatt, 2002; Regehr, Stern, Shlonsky, 2007). Indeed, EBP explicitly calls for evidence to be balanced with other sources of knowledge such as clinical expertise and client values (Gilgun, 2005). The pressing question is whether this balance is occurring at all—that is, is evidence collected during structured assessment being integrated into treatment decision making in a manner consistent with the EBP framework? The scant evidence base suggests that it may not be (Garland, Kruse, \& Aarons, 2003; Miller \& Maloney, 2013).

Despite the centrality of standardized assessment to EBP and its critical role in helping the practitioner identify relevant ESIs, the subject has received minimal attention in the scholarly and professional literature. Mash and Hunsely (2005) recognized and acknowledged this oversight far earlier than most; their observation is worth reproducing verbatim: 
It seems self-evident that assessments should be 'useful' in designing and evaluating effective and efficient services for children and families. ...

Nevertheless, although their importance is widely acknowledged, the nature and strengths of the links between assessment and intervention remain tenuous at best, and the role of assessment in EBT [evidence-based treatment] is virtually unexplored. (p. 363)

Surprisingly, little has changed in the span since Mash and Hunsely (2005) voiced this assertion. Writing almost a decade later, Grady \& Drisko (2014) echoed an identical concern, noting that assessment is "neglected or very minimally addressed in the teachings, writings, and explanations of EBP (p. 5). In social work (Ponniah et al., 2011), as well as other behavioral health disciplines (e.g., clinical psychology—Barlow, 2005), the vast majority of EBP scholarship has focused on intervention rather than assessment. That is to say, research attention has been almost exclusively focused on identifying and testing effective interventions with very little exploration of the process wherein client needs are systematically identified and linked with appropriate treatments or services. The purpose of the proposed study is to explore this neglected line of inquiry as manifested in one practice context—child welfare.

\section{Assessment in the child welfare context.}

In 2015, child protective services conducted an investigation or provided an alternative response for more than 3.4 million children in the United States; more than 600,000 of these children were identified as victims of maltreatment and almost 150,000 of them entered foster care (U.S. Department of Health \& Human Services, 2017a). 
There are approximately 400,000 children in out of home care on any given day (U.S. Department of Health \& Human Services, 2017b). In Kentucky—-the site of the present study — more than 8,500 children are currently residing in an out of home placement (Kentucky Cabinet for Health \& Family Services, 2018). Children involved with the child welfare system often have extensive trauma histories (Greeson et al., 2011; Kisiel, Fehrenbach, Small, \& Lyons, 2009). It has long been recognized that these traumatic experiences and their associated effects on emotional and behavioral wellbeing may be unrecognized, overlooked, or untreated (Ko et al., 2008).

In the past two decades there has been increased pressure on health care and human service systems — including child welfare — to improve services by adopting empirically-evaluated and evidence-based models and practices (Lang, Randall, Delaney, \& Vanderploeg, 2017). Concurrently, the critical importance of improving child welfare screening and assessment practices has been increasingly accepted (Conradi, Wherry \& Kisiel, 2011; Kisiel, Conradi, Fehrenbach, Torgersen, \& Briggs, 2014; Romanelli et al., 2009). It is now being recognized that these two initiatives can be merged to improve services for children and families involved with the child welfare system. For example, Saunders (2015) has called for evidence-based service planning: conducting sound assessments, identifying measurable intervention goals from these assessments, using critical thinking skills to select the most effective interventions, and then monitoring progress toward such goals. 


\section{Assessment in Project SAFESPACE.}

Kentucky has recently adopted a process to improve service planning and delivery akin to that described by Saunders (2015). Initiated in 2013, Project SAFESPACE is a collaborative initiative among the University of Louisville, the Kentucky Cabinet for Health and Family Services (CHFS), Eastern Kentucky University Training Resource Center, and the Kentucky Partnership for Children and Families. The project is intended to improve the early identification and effective treatment of trauma and behavioral health needs of child welfare involved youth.

Project SAFESPACE initiated systematic screening and assessment for all children entering out of home care in Kentucky. Upon entry into care or other qualifying event (e.g., placement disruption) a child is assessed for trauma exposure and behavioral health needs using age-appropriate standardized screening instruments. The screening is conducted by a Department of Community Based Services (DCBS) social services worker within 10 days of the qualifying event. If the screening instruments indicate that a more thorough assessment is warranted (i.e., the child "screens in"), the child is referred to a service provider who is tasked with completing an initial functional assessment within 30 days of placement as well as a reassessment every 90 days in care.

Project SAFESPACE selected the Child and Adolescent Needs and Strengths (CANS) as its statewide functional assessment instrument. The CANS assessment does not generate a clinical diagnosis or specific treatment prescriptions. Rather, the instrument is intended to "facilitate the linkage between the assessment process and the design of individualized service plans including the application of evidence-based practices" (Praed Foundation, 2016, p. 4). The CANS assessment's intended usage 
directly reflects the role of structured assessment in the broader EBP process as outlined above. Moreover, it aligns with one of the primary goals of Project SAFESPACE: to promote the use of assessment-driven, evidence-based treatment for children in out-ofhome care. This study explores the degree to which treatment practices are indeed assessment-driven and consistent with EBP by investigating the relationship between responses on the CANS functional assessment and the treatment modality subsequently prescribed by the assessing clinician.

\section{Theoretical Framework}

As previously explicated, the evidence-based practice process is predicated upon certain assumptions about the practitioner's epistemic process. That is, EBP assumes that practitioners will evaluate, sort, and prioritize relevant information (e.g., research evidence, assessment data) and then use this information to optimize their decisions and practices (Webb, 2001). As such, one's understanding of EBP is grounded in presuppositions about decision making. Writing from a medical perspective, Spring (2008) has described decision making as the "lynchpin" of evidence-based practice.

Given the centrality of decision making to the EBP process, decision making theories can provide a useful framework for investigating the clinical decision making processes of mental health professionals (Baker-Ericzen, Jenkins, Park, \& Garland, 2015).

Historically, EBP has been most directly associated with the classical or rational choice decision making tradition (van de Luitgaarden, 2009; Webb, 2001; White \& Stancombe, 2003). As envisioned by rational choice theory, a decision is rational if it meets four criteria: (1) based on the decision maker's current assets (e.g., resources, 
physiological state, psychological capacities), (2) based on possible consequences of the choice, (3) based on evaluated likelihood (as governed by probability theory) when consequences are uncertain, and (4) adaptive within the constraints of probabilities and the values associated with each possible consequence of the choice (Hastie \& Dawes, 2010). As implied by these criteria, rational decision making theories are focused on decision events - the point at which a decision maker "surveys a known and fixed set of alternatives, weighs the likely consequences of choosing each, and makes a choice" (Orasanu \& Connolly, 1993). Moreover, decision events are grounded in demanding informational requirements. That is to say, before making a choice the decision maker must engage in a deliberate and analytic process (i.e., appraising current assets, weighing alternative choices, evaluating contingencies) that necessitates a relatively comprehensive information search (Lipshitz, Klein, Orasanu, \& Salas, 2001).

One can readily recognize the implicit connection of rational decision making principles to the EBP process; van de Luitgaarden (2009) has gone so far as to describe EBP as "an operationalization of a rational choice approach to judgment and decision making” (p. 244). Conceptualized thusly, the clinician-facing client problem A-draws upon the best available evidence to consider the relative probability that intervention $\mathrm{B}$ compared to intervention $\mathrm{C}$ or intervention D will lead to desired outcome E. In effect, the clinician is assumed to operate in a quantitatively-oriented, statistically-minded mode of clinical decision making (van de Luitgaarden, 2009).

The degree to which clinicians' decision making actually reflects this notional process of rational choice has been challenged in the scholarly literature. For example, Webb (2001) has argued that "the evidential-based model is a mechanistic approach 
which regards social workers largely as 'information processors' operating within closed systems of decision making" when, in fact, social workers operate within the bounds of changing legal and organizational requirements and "will tend to fall back on inclinations, values and common sense when making decisions" (p. 67). Proponents of the EBP model have countered such arguments by noting that EBP arose from the recognition that "professionals are not rational agents and that in spite of intentions of professionals to provide competent, ethical services informed by practice-related research, they do not do so" (Gibbs \& Gambrill, 2002, p. 463). Because professionals are not guaranteed to make the rational choice, the EBP framework acknowledges the uncertainty and bias that might impact practitioner decision making and thus encourages rigorous evaluation criteria when appraising the available evidence (Gibbs \& Gambrill, 2002).

It would seem that both sides of the debate acknowledge that — despite the similarities and shared intellectual lineage-EBP is not dependent upon a rational choice conceptualization of decision making. The question thus arises, "can the basic propositions and processes of EBP be supported by an alternative understanding of practitioner decision making?" The theory of naturalistic decision making (NDM) has been proposed as a viable alternative to the rational choice theory for conceptualizing, implementing, and evaluating evidence based practice (Baker-Ericzen, Jenkins, Park, \& Garland, 2015; Falzer, 2004). 


\section{Naturalistic Decision Making}

The naturalistic decision making approach emerged in response to the realization that "researchers were not likely to find out how people actually made decisions by conducting experiments to test hypotheses derived from statistical and mathematical models of ideal choice strategies" (Klein, 2008, p. 456). In contrast to the classical rational decision making researchers, NDM researchers were not only interested in the decision event itself but also in the human decision maker within the natural decision making setting (Lipshitz et al., 2001; Orasanu \& Connolly, 1993). They sought to explore the strategies that individuals used to make difficult decisions in complex situations. For example, Klein, Calderwood, and Clinton-Cirocco (1986) investigated the decision making processes of firefighters and O'Hare (1992) examined those of aircraft pilots. Early NDM research provided compelling evidence that individuals, when faced with decisions in "real world" settings, were not identifying and comparing a set of options but rather were drawing on an experience-based schema to rapidly assess, categorize, and select a course of action (Klein, 2008).

In 1997, Zsambok offered the following concise definition of NDM: "the way people use their experience to make decisions in field settings" (p. 7). As opposed to decisions made within the laboratory conditions traditionally favored by rational decision making researchers, decisions made in a naturalistic setting are complicated by a number of factors including ill-structured problems, dynamic environments, shifting or competing goals, action/feedback loops, time stress, high stakes, multiple players, and organizational goals and norms (Orasanu \& Connolly, 1993). As such, field settings "establish the 
eliciting conditions for making decisions and shape decisions through their constraints and affordances" (Lipshitz et al., 2001, p. 334).

The human (and thus rationally-bounded) decision maker is of central importance in the NDM framework. The ideal, omniscient, logically-consistent decision maker posited by classical decision theory has little relevance in the real world (Beach \& Lipshitz, 1993). By contrast, NDM presupposes that decision makers are shaped by prior experience and content knowledge and seeks to identify those who are proficient and demonstrate expertise (Lipshitz et al., 2001). Within the NDM framework, the quality of the decision making process is judged not by its procedural rationality or logical consistency but rather by the quality of the decisions produced by the process (Bordley, 2001).

Lipshitz and colleagues (2001) have distilled four essential characteristics of the NDM framework:

(1) Process orientation - NDM models describe what information decisionmakers seek, how they interpret this information, and which decision rules they actually use.

(2) Situation-action matching decision rules - NDM theories conceptualize decision making as a matter of matching rather than of choice.

(3) Context-bound informal modeling - Within the NDM framework there is little use for formal, abstract models; rather decision maker knowledge is recognized as context-specific and sensitive to both semantic and syntactic content.

(4) Empirical-based prescription - NDM theorists recognize that "prescriptions which are optimal in some formal sense but which cannot be implemented are 
worthless" (p. 335). Rather, decision making prescriptions are derived from descriptive models of expert performance.

\section{Application of naturalistic decision making theory to the present study.}

Clearly, treatment decision making within the CW service environment does not happen in a vacuum; if the decision event itself (i.e., the assessing clinician's prescription of a treatment modality) is considered in isolation, one cannot account for the potential impact of contextual factors (e.g., time stress, organizational goals and norms) on the decision making process. Naturalistic decision making theory provides a compelling conceptual framework for the investigation of treatment decision making within a complex and dynamic service system.

Each of the four NDM essential characteristics has implications for the present study's line of inquiry. The first characteristic — process orientation — reinforces the study's purpose. NDM recognizes that just because a source of information is available it does not necessarily follow that this information is incorporated in the decision making process. Thus, NDM-based models "have to describe what information decision makers actually seek" (Lipshitz et al., 2001). At the broadest level, this study seeks to investigate the degree to which the evidence suggests that assessment-derived information is incorporated in the clinician's treatment decision making process.

The second characteristic — situation-action matching rules — is particularly salient to the present inquiry's methodological approach. The term matching suggests that "decisions are made by sequential evaluation of alternatives in terms of appropriateness to the situation" (Lipshitz, 1994, p. 49). Instead of decisions framed by choosing among 
alternatives (as in classical decision making frameworks), matching relies on situational assessment; potential options are selected or rejected not in relation to one another but based on their perceived compatibility to the situational context. NDM proposes, bolstered by research evidence (e.g., Beach, 1993), that decision makers rarely consider every possible choice. Rather, as soon as mental simulation identifies one "good enough" option it is likely to be selected without an exhaustive consideration of other alternatives (van de Luitgaarden, 2009). This mental matching process may be deliberate or nondeliberate, explicitly analytic or reliant upon pattern matching and informal reasoning (Klein, 1998; Lipshitz, 1994; Lipshitz et al., 2001).

Applied to the treatment decision making process, the NDM framework suggests that clinicians may be less concerned with weighing all possible interventions against one another in light of the available evidence, but rather ask themselves, "Given situation A, is intervention B appropriate?" If intervention B is deemed sufficient given the available information, interventions C and D may never even be mentally appraised. Reflecting this theoretical proposition, the analytic approach employed in the present study examines the extent of the "match" (e.g., predictive strength and explained variance) between the structured assessment and the subsequently prescribed intervention.

The third characteristic_context-bound informal modeling — frames the study's modeling process. NDM has shifted the conception of decision making away from domain-independent, abstract general models to domain-specific, knowledge-based applied models (Klein, 2008; Lipshitz et al., 2001). As such, this is not a theory-testing study. That is to say, while NDM theory serves as a conceptual framework, this study does not derive testable research hypotheses from a formal decision making model. 
Rather, the proposed models are assumed to reflect the applied decision making process of clinicians within a particular context and in relation to particular information.

Finally, the fourth characteristic of the NDM framework—empirical-based prescription - informs the interpretation and implication of study findings. Whereas rational choice theory entails normative prescription, description precedes prescription in the NDM framework (van de Luitgaarden, 2009). That is to say, NDM theorists accept "that 'ought' cannot be divorced from 'is"” (Lipshitz et al., 2001, p. 335). As such, descriptive findings regarding decision making behavior within a given context are not only recognized as useful but are indeed a necessary first step in any effort to enhance decision makers' performance. Thus, this novel study can be seen as an exploration of clinical decision making as it currently is; from this, one can better gauge what improvements are feasible and ground them in concrete demonstrations of performance.

\section{Importance of the Present Study}

The present study has important implications for social work research, education, and practice. As noted, the role of assessment in evidence-based practice has been largely neglected in the scholarly literature (Grady \& Drisko, 2014). As such, this study provides new empirical data about an issue that has received little research attention. Findings establish a foundation for an ongoing research program by indicating the degree to which treatment planning reflects client needs as identified through structured assessment. Moreover, by invoking an NDM framework, this study offers a conceptual mechanism whereby the empirical data can be meaningfully (albeit tentatively) contextualized within an understanding of EBP that is not limited by classical decision making assumptions. 
In terms of social work education, the study findings provide valuable insight regarding the educational needs of clinical social work students. The persistent "chasm" between research and practice in social work has been well documented (BledsoeMansori et al., 2017) and evidence suggests that many clinicians entering the workforce are not sufficiently prepared to engage in evidence-based practice (e.g., Barwick, 2011). A decade ago Walker and colleagues (2007) observed: "social work education needs to make sure that all social workers... understand how to use EBP to select an EST" (p. 368); this study will explore the degree to which this educational imperative has been satisfied. Emerging research suggests that practitioner knowledge is among the most important predictors of engagement in EBP or the use of ESIs (Tuten et al., 2016). Given the conceptual confusion and definitional ambiguity regarding the EBP process, there is little wonder that social work students may not feel confident in their capacity to integrate structured assessment into such a framework. This study will help social work educators gauge current strengths or shortcomings in the professional development of social work clinicians so as to develop innovative and targeted educational strategies.

In the child welfare practice environment, an accurate and comprehensive assessment is critical for the identification of a child's existing strengths and treatment needs (Rosanbalm et al., 2016). After years of being "overlooked and understudied," structured assessments are increasingly being recognized as an essential component in the treatment of children in out of home care (Igelman et al., 2007, p. 17). However, the effort to incorporate structured assessment into professional practice in such a way as to inform evidence-based service delivery is fragmented and incomplete (Milne \& CollinVezina, 2015). This study explores the use of structured assessment to inform evidence- 
based treatment planning within a redesigned system of care. Findings provide insight into whether systematic assessment is influencing treatment planning in a manner consistent with what would be expected in an EBP framework. The findings have direct practice implications in terms of ongoing training, supervision, and evaluation objectives. 


\section{CHAPTER 2:}

\section{REVIEW OF THE LITERATURE}

The present study is situated at the intersection of several distinct areas of scientific inquiry including treatment decision making, clinical assessment, and evidencebased practice. Consequently, there is a diverse array of relevant literature to consider. The following literature review is structured into two primary sections. In the first section, treatment decision making publications are discussed. Coupled with EBP (as discussed in the first chapter), treatment decision making provides a conceptual grounding for the investigation of ESI selection. A review of the literature will help contextualize the present study within the broader field of research that has sought to understand how clinicians engage in treatment planning. In the second section, focus shifts from the conceptual to the concrete; the studies reviewed in this section are directly related to one more elements of the present study's research method (e.g., the CANS functional assessment).

\section{Conceptually-Relevant Literature: Treatment Decision Making}

Understanding the processes whereby practitioners identify, select, and employ specific therapeutic interventions must be a central concern in efforts to improve the dissemination and sustainability of effective treatment practices (Cook, Schnurr, Biyanova, \& Coyne, 2009). As Eells and Lombart (2003) note: "It is axiomatic that how a psychotherapist thinks and makes decisions about patients will affect the treatment 
process and outcome" (p. 187). While this observation may be axiomatic, it belies a complex and poorly understood web of influences, mechanisms, and decision events. The simple question "how do therapists select the best intervention for their clients?" has proven exceedingly difficult to answer despite decades of research attention.

In 1997, Witteman and Kunst observed that the process of treatment planning had not yet been adequately analyzed and no formal models of treatment decision making had been developed. The slow growth of academic knowledge in this area is evidenced by the declaration ten years later that clinician decision making research was "in its infancy" (Schottenbauer, Glass, \& Arnkoff, 2007, p. 225) and again more than a decade after that (Gutierrez, Fox, Jones, \& Fallon, 2018, p. 95). Nevertheless, some progress has been realized. While a comprehensive treatment decision making model is still lacking, a diverse body of research that spans national and disciplinary borders has identified a number of factors that influence treatment planning. These studies can be categorized by the design employed to examine treatment decision making: (1) survey or questionnaire, (2) case study or vignette, and (3) naturalistic setting with actual clients.

\section{Treatment decision making studies using a survey or questionnaire design.}

The studies reviewed in this category are methodologically unsophisticated but straightforward. They approach the question of treatment planning by directly asking clinicians - via survey or questionnaire — about the factors that influence their decision making when considering an intervention. While this type of design is poorly suited for the development of a process model, it has been effective in identifying the salient factors that practitioners consider before selecting a treatment strategy 
Lucock, Hall, and Noble (2006) surveyed qualified psychotherapists ( $\mathrm{n}=95)$ and psychologists in clinical training $(n=69)$ to identify the primary influences on their clinical practices. Data was collected using the Questionnaire of Influencing Factors on Clinical Practice in Psychotherapies (QuIF-CliPP) consisting of 39 items structured into four categories: training, literature, practice, and personal factors. Responses indicated that qualified psychotherapists were most influenced by psychological formulation, current supervision, post-qualification training, and client characteristics. Clinical psychologist trainees were most influenced by current supervision, professional training, psychological formulation, and client characteristics. Neither group rated any literaturerelated factors (i.e., treatment manuals, evidence-based practice guidelines, journal articles) as highly influential.

However, when responses were analyzed by therapeutic orientation, it was found that practitioners with a cognitive-behavioral background reported being more influenced by evidence-based practice guidelines and research-based journal articles than other orientations. By contrast, therapists with a psychodynamic, person-centered, integrative, or elective orientation were most influenced by intuition or judgment when making treatment determinations. Altogether, Lucock and colleagues' (2006) findings indicate that while the surveyed practitioners are not greatly influenced by research evidence, their treatment decision making is heavily influenced by client-level factors (i.e., psychological formulation, client characteristics, client feedback).

As part of a larger publication discussing the need for improved theory and research regarding to the decision making processes of integrative psychotherapists, Schottenbauer, Glass, and Arnkoff (2007) conducted a preliminary study exploring one 
dimension of treatment decision making among 171 practicing psychotherapists (including psychologists, social workers, psychiatrists, and counselors). Using an online questionnaire to collect data, the researchers asked participating clinicians how they would adjust their treatment planning in the case of a client who was not experiencing noticeable improvement or treatment gains. The most common responses among participants included reassessment of the client, reassessment of the client's environment or motivation, or reconceptualization of the client's problems. Interestingly—even among practitioners identifying as integrative or eclectic—substantially fewer respondents indicated that they would make a change to the current treatment plan or endorsed a specific treatment modality (e.g., cognitive-behavioral) that could be employed in a renewed attempt to elicit client growth.

Nelson and Steele's (2008) study examining the relative importance of various factors influencing treatment selection is among the most heavily cited publications in the treatment decision making literature. Via an online survey, 206 mental health practitioners - including psychologists and social workers - were ask to rate 29 potential considerations in terms of their likelihood to influence treatment selection. They were also asked to rank (in order of relative importance) ten broader characteristics that might influence treatment planning.

Practitioners reported that their decisions to employ a particular treatment were most heavily influenced by empirical support, flexibility, colleague recommendation, and appeal to clients. In terms of relative rankings, flexibility emerged as the most important characteristic in considering the use of a treatment. The second most important characteristic was that the treatment was supported by evidence. Interestingly, while 
practitioners rated empirical support as the most influential factor in treatment selection and ranked research support second only to flexibility in terms of relative importance, a treatment's inclusion on a list of "empirically-supported" or "evidence-based" treatments was among the lowest rated decision making considerations.

Cook and colleagues (2009) conducted an internet-based survey of 2,607 psychotherapists to identify the factors with the greatest influence on practice behaviors. Respondents indicated that decisions to adopt a new treatment approach were most heavily influenced by ease of integration with existing practice, endorsement by respected therapists, and accessible training opportunities. By contrast, positive findings reported in research journals, endorsement by a professional organization as being evidence-based, and client testimonials regarding effectiveness were the least influential factors.

As is evident, findings from questionnaire or survey research have been mixed. For example, based on the work of Lucock et al. (2006) and Cook et al. (2009) one might conclude that research evidence or empirical support is not a significant consideration for clinicians selecting a treatment approach. However, Nelson and Steele's (2008) findings challenge this conclusion, indicating that evidentiary support is among the practitioner's most important concerns. Nonetheless, there are points of agreement. For instance, three of the studies identified colleagues and/or supervisors as important factors influencing treatment decision making (Cook et al., 2009; Lucock et al., 2006; Nelson \& Steele, 2008). Perhaps most relevant to the present study, the available evidence does consistently suggest that client-level factors (such as might be identified by a functional assessment) are influential factors in the initial treatment planning process (e.g., Lucock 
et al., 2006; Nelson \& Steele, 2008) as well as when confronted with stagnated clinical growth (Schottenbauer et al., 2007).

\section{Treatment decision making studies using a vignette or case study design.}

Most of the treatment planning research to date has made use of case studies or vignettes to investigate clinical decision making. Rather than simply asking practitioners about potentially influential factors (as in the survey-based research described above), these studies present the clinician with a notional case or cases and then analyze the process of selecting an appropriate treatment.

Recognizing that little was known regarding the cognitive processes of clinicians engaged in treatment planning, Witteman and Kunst (1997) conducted a process-tracing study to gain insight into the "nature and sequence of the considerations that psychotherapists have when they are asked to propose a treatment for a depressed patient" (p. 157). The researchers tested the hypothesis that "therapists reach their treatment decisions by interpreting the case, by associating a therapy option with this interpretation and by substantiating this option to their satisfaction through a focus on confirming information" (p. 159) To test this hypothesis, eleven clinical psychologists of different therapeutic orientations were presented with a case study of a depressed client and asked to think aloud about how they would construct a treatment plan.

After coding and analyzing the transcripts of the proposed treatment plans, Witteman and Kunst (1997) concluded that their hypothesis was supported. That is to say, all participants offered an interpretation of the case, proposed a treatment, and then selectively identified information to confirm this treatment option without considering 
alternatives or potentially disconfirming information. Given their findings, the researchers posited that rather than considering "what would be the most suitable treatment plan for this patient?", the therapists were actually most concerned with "could I treat this patient with the methods I usually apply?” (p. 168). Surprisingly, when the researchers shared their findings with study participants and other practicing clinicians, the therapists agreed that their treatment decisions were often quite unstructured and subjective.

Witteman and Koele (1999) later expanded on Witteman and Kunst's (1997) exploratory findings by investigating the explicit and implicit explanations of clinical treatment recommendations among a larger sample $(n=56)$ of registered psychotherapists. The participating psychotherapists were asked to read four case descriptions and answer questions regarding their proposed course of treatment. This process was repeated twice over the course of one month- once with the therapists answering open-ended questions about treatment and once using a structured questionnaire that listed potential symptoms and treatment options. These two sets of responses were then coded and compared.

Findings indicated that the best (although weak) predictor of treatment decisions was the therapist's theoretical background (i.e., psychodynamic). When compared, responses from the unstructured and structured case questionnaires were only marginally related to one another and only four participants proposed the same treatment on their first (unstructured) and second (structured) responses. Moreover, there was little agreement among the participants as to which symptoms were most relevant for each case. Witteman and Koele (1999) concluded that treatment proposals were not based 
directly on client data or case information but instead on "a schema or schemas that go with certain theoretical orientations, refined by practical experience" (p. 110). The researchers acknowledged that their findings lead to additional questions: "Do psychotherapists in practice actually actively decide upon a specific treatment method after substantive processing, before they start treatment? Or is it maybe more a matter of deciding by recognition, an almost automatic process?” (p. 111).

Eells and Lombart (2003) explored case conceptualization and treatment planning among a sample $(\mathrm{n}=56)$ of cognitive-behavioral and psychodynamic therapists. The researchers recruited clinicians of three types: novice, expert, and experienced. Each therapist was presented with six vignettes representing three common psychiatric disorders; after reviewing the vignettes, the participants completed a questionnaire describing their understanding of the cases and proposed treatment strategies. The researchers sought to explore differences in case formulation and treatment prediction among the three experience categories as well as between the two therapeutic orientations.

In terms of case formulation, Eells and Lombart (2003) found that cognitivebehavioral therapists focused most heavily on symptoms and problems while those with a psychodynamic orientation placed more emphasis on childhood history, coping mechanisms, strengths, and treatment obstacles. When asked about the effectiveness of treatment, cognitive-behavioral therapists anticipated greater improvement than did psychodynamic therapists. In terms of initial treatment planning, psychodynamic therapists in general and expert clinicians within either orientation predicted a need for longer treatment with more frequent sessions. 
Falvey, Bray, and Hebert (2005) investigated the process of treatment planning among a small sample $(n=25)$ of mental health professionals including psychologists, social workers, and counselors. Participants were administered the Clinical Treatment Planning Simulation: Case 1-B (CTPS) — a notional case representing a client with ADHD. After reviewing the case materials (e.g., psychosocial history, client interview, parent interview), the clinicians were instructed write a case conceptualization and develop a treatment plan. Upon completing these clinical tasks, the participants completed a structured follow-up interview with the research team. The researchers then used a process-tracing strategy to identify the problem-solving styles and clinical judgment strategies employed by the clinicians. This was followed by the development of a classification scheme to predict case conceptualization and treatment planning performance.

Falvey and colleagues (2005) identified four treatment-planning clusters. The first cluster was representative of a template approach; these clinicians relied on direct diagnostic matching to inform their treatment planning. The second cluster was representative of a novice approach as evidenced by a lack of any noticeable cognitive schema for case evaluation or planning. Clinicians using the novice approach relied on heuristic strategies to plan treatment. The third cluster was representative of a mastery approach; these clinicians produced thorough case reviews in which supporting and disconfirming evidence was carefully weighed against initial hypotheses. Clinicians employing a mastery approach ultimately produced comprehensive, client-based treatment plans. Finally, the fourth cluster was representative of an efficient approach. 
The efficient approach was characterized by rapid assessment and seeking direction from others involved in the case (e.g., parents, teachers).

Kwaadsteniet and colleagues (2010) used a cognitive mapping approach to examine intervention selection among a sample $(n=40)$ of Dutch clinical psychologists. Study participants were presented with two case studies (including the results of a number of psychological tests) involving two depressed children. After reviewing the case materials, the clinicians were asked to complete two tasks. One task entailed the construction of a causal map in four steps: (1) identification of problems and hypothesized causal factors, (2) rating of problems in terms of potential harm or distress, (3) arrangement of problems and causal factors into causal pathways, and (4) rating of the modifiability of each causal factor or problem. The other task involved selecting the five most effective treatments for each case from a list of ten possible interventions (e.g., individual cognitive therapy, family therapy, social skills training) and ranking these interventions in terms of expected effectiveness. Half of the sample completed the causal mapping task first, while the other half completed the intervention selection task first.

When the psychologists' causal maps were compared, Kwaadsteniet and colleagues (2010) observed low levels of agreement about the causal factors and relationships underlying the two case studies. However, individual clinician's ratings of intervention effectiveness could be significantly predicted from his or her own causal model. As Kwaadsteniet and colleagues (2010) effectively summarized, these findings indicate "that different causal models lead to different ratings of intervention effectiveness, and as a consequence may lead to different intervention choices by different clinicians for the same client” (p. 588). 
Groenier, Pieters, Witteman, \& Lehmann (2014) investigated the degree to which problem complexity influences case formulation and subsequent treatment decision making. A sample $(\mathrm{n}=211)$ of Dutch psychologists was presented with two case vignettes - one with a simple problem presentation and one of greater clinical complexity. After reviewing each vignette, the participants were asked to: (1) select the most likely DSM-IV classification, (2) describe in their own words the etiology of the client's problems, and (3) select one or two interventions from a list of 18 specific treatment methods.

Results indicated that the study participants formulated higher quality case formulations for the less complex case than for the more complex case. Interestingly, the treatment methods selected by the psychologists were neither associated with the DSMIV classifications they identified nor related to the hypothesized cause of the clients' problems. Rather, treatment decisions were most highly associated (although still weakly) with clients' pattern of complaints as described in the case materials. These findings were interpreted as consistent with Witteman's (Witteman \& Koele, 1999; Witteman \& Kunst, 1997) earlier research suggesting that treatment selection is primarily schema-driven.

Baker-Ericzen and colleagues (2015) used case vignettes to investigate assessment and treatment formulations among a sample $(n=48)$ of pediatric clinicians including psychiatrists, psychologists, social workers, and marriage and family therapists. This research is particularly relevant to the present inquiry as it is the only treatment decision making study to date to draw explicitly upon NDM theory. Participating clinicians were grouped based on prior training in a specific evidence based treatment. Using a "think aloud" technique, clinicians verbalized their case conceptualization and 
offered a treatment decision in response to a randomly presented vignette. Researchers coded responses by applying five primary decision making processes identified in the NDM literature: (1) type of reasoning, (2) organization of information, (3) attention to information and level of abstraction, (4) finding solutions, and (5) incorporating actuarial information and flexibility in application.

Baker-Ericzen and colleagues' (2015) results indicate that clinicians with prior training in one or more EBTs demonstrated clinical decision making skills consistent with NDM's conceptualization of expert performance (e.g., forward reasoning, comprehensive treatment planning). The researchers posited that training in an EBT might in and of itself improve treatment decision making skills: "The process of EBT training may also teach meta-cognitive skills such as attention to relevant cues, organizational skills, and gist formulation so that the experience of EBT training may generalize to improved decision making skills which can be applied to ne, complex cases" (p. 150). Importantly, this study demonstrates the viability of using NDM theory to investigate treatment decision making within an EBT/EBP context.

Most recently, Gutierrez, Fox, Jones, and Fallon (2018) explored the treatment planning process of a small sample $(n=9)$ of experienced counselors. The researchers presented each participant with a ten-minute video vignette of a client discussing her clinical concern (family conflict and stress). While watching the vignette, the counselors were asked to think aloud concerning their initial impressions and treatment strategy. Upon completion of the video, the researchers conducted a semi-structured interview with each counselor to elicit additional information. The clinician's verbalized thoughts and interview responses were then transcribed, coded, and qualitatively analyzed. 
Gutierrez and colleagues (2018) did not attempt to appraise the relevance or adequacy of the treatment selections. Rather, they focused solely on the process whereby such decisions were reached. The researchers identified four subsequent domains through which the treatment planning process progressed. The first domain, assessment steps, involved the exploration of information that the counselor needed to determine which treatment would work best (e.g., identifying unhealthy behaviors). The second domain, clinical impressions, consisted of those facets of the client's presentation that the counselor found to be clinically significant (e.g., client strengths). The third domain, treatment factors, entailed the consideration of factors that might influence the final treatment determination (e.g., family dynamics, client readiness). Finally, the domain of treatment strategies referred to the identification and selection of specific treatment techniques deemed most appropriate for use with the client.

Four of the vignette-based studies reviewed above have involved the Dutch scholar Celia Witteman, who was among the first to investigate the subject of treatment decision making and remains the most prolific author in the field. (Groenier, Pieters, Witteman, \& Lehmann, 2014; Kwaadsteniet, Hagmayer, Krol, \& Witteman, 2010; Witteman \& Koele, 1999; Witteman \& Kunst, 1997). These studies evidence a systematic and progressive research program that has produced compelling empirical evidence that the process of treatment selection cannot be explained by any particular factor or combination of factors - clinician's theoretical background, client characteristics, suspected etiology of the problem, hypothesized causal mechanisms - but rather are mediated by an underlying schema. This schema is shaped by clinical orientation and tempered by practice experience; it does not appear to be altogether logical (Witteman \& 
Kunst, 1997) and almost certainly involves some degree of subjectivity and bias

(Kwaadsteniet et al., 2010). Applying this proposition to the current study suggests that the observed relationship between assessment responses and ESI prescriptions may not be as direct or strong as one would expect based on the traditional rational choice theory of EBP. However, EBP — as viewed through the lens of NDM—does not necessitate such a unidimensional treatment decision making mechanism.

\section{Treatment decision making studies in a naturalistic setting.}

Only two treatment decision making studies have made use of actual clients in a naturalistic setting to examine clinician treatment selection. Zuber (2000) investigated the relationship between a client's own problem formulation and subsequent psychotherapeutic treatment recommendations. Zuber's (2000) sample consisted of 159 Swedish patients receiving public financial support for psychotherapy; all had undergone an extensive clinical interview and assessment process which included an opportunity for the client to describe clinical concerns in his or her own words. These were coded into three broad categories: (1) problems related to relationships, (2) problems related to symptoms, or (3) mixed problems. Upon completion of the clinical interview and review of the client's diagnoses and problem formulation, a psychologist (as part of a qualification team) would recommend the patient to a specific psychotherapist. The orientation of the psychotherapist to which the client was referred was used to classify treatment recommendations.

Zuber (2000) found that clients who formulated their problem as relational in nature were most frequently recommended to insight-oriented psychotherapies (e.g., 
psychodynamic therapies, expressive therapies) while those whose problem formulations focused on symptoms were more likely to be recommended to directive-oriented psychotherapies (e.g., cognitive-behavioral therapies). Furthermore, with the sole exception of anxiety disorders, clients' problem formulations were found to have more influence on treatment recommendation than did formal psychiatric diagnoses. Zuber's (2000) findings suggest that a client's own problem formulation may have a substantial effect on the clinician's treatment planning process.

Scheidt and colleagues (2003) investigated the treatment selections of a sample of 24 German private-practice psychotherapists who assessed a total of 238 potential clients during the study period. After completing one or more clinical interviews, the psychotherapist would determine whether or not to accept the individual as a client. The researchers also recorded the type (i.e., psychodynamic or psychoanalytic) and duration (i.e., long-term or short-term) of psychotherapy that was prescribed.

Scheidt and colleagues (2003) findings indicate that client motivation was the most important factor in determining whether a client was accepted for treatment and the therapist's personal response to the client (e.g., client aroused therapist's interests, therapist was emotionally touched by client) contributed the most to treatment decisions. Though the therapists had access to information regarding client diagnosis, symptom severity, and areas of clinical concern, these factors contributed very little to the process of treatment selection. Given this unexpected finding, Scheidt and colleagues (2003) called for more research linking standardized assessment with treatment planning among psychotherapists - importantly, this is precisely the aim of the present study. 


\section{Summary of the conceptually-relevant literature.}

While there is a modest body of scholarly literature regarding treatment decision making, synthesizing this research is particularly challenging. Samples have been comprised of a variety of professional disciplines, including psychotherapists, psychiatrists, psychologists, counselors, and clinical social workers. Some samples have been homogenous in terms of professional background (e.g., counselors - Gutierrez et al., 2018) while others have included a mixture of disciplines (e.g., Falvey et al., 2005). The settings from which these samples were drawn are just as varied: German private practice (Scheidt et al., 2003), United Kingdom National Health Service clinics (Lucock et al., 2006), Dutch psychotherapy institutes (Witteman \& Kunst, 1997), American community mental health centers (Falvey et al., 2005). Without exception, each sample was selected using a nonprobablistic strategy—typically purposive or convenience sampling.

Likewise, the operationalization of the concept treatment decision is not consistent among all studies. For example, Kwaadsteniet and colleagues (2010) examined participants' selections from a list of specific interventions (e.g., individual cognitive therapy) while Scheidt and colleagues (2003) focused on a binary choice between longor short-term psychotherapy. In the case of Zuber (2000), the theoretical orientation of the psychotherapist (e.g., psychodynamic) served as a proxy for treatment selection. Some researchers (e.g., Gutierrez et al., 2018) were not interested in the appropriateness of a treatment selection but only the process whereby it was reached; others (e.g., Witteman \& Koele, 1999) employed complex designs to analyze the congruence between the case information and the clinician's selected treatment plan. 
Given these substantial limitations, one must exercise great caution in drawing any overarching conclusions about treatment decision making other than those of the most general nature. The current best evidence does suggest that clinicians' treatment decisions are influenced by a number of factors - both internal (e.g., theoretical orientation) and external (e.g., client characteristics) and that a (possibly subjective) schema mediates the interaction between these two realms. It is important to note that this conjecture is entirely consistent with the EBP model, which also recognizes that practice in informed by factors other than empirical evidence (e.g., clinical expertise). Moreover, the findings that suggest that clinical decision making is derived from an experiencedbased schema rather than a deliberate weighing of potential choices are directly reflective of the NDM framework even if they do not explicitly draw upon the theory. Whether viewed through the lens of EBP or through the nascent understanding of treatment decision making grounded in the research reviewed above, one would have little justification for hypothesizing that functional assessment responses can account for all of the observed variation in treatment planning (i.e., ESI selection). Rather, the more important question may be whether it accounts for any and, if so, how much?

\section{Concretely-Relevant Literature: Assessment and Empirically-Supported Intervention}

The empirical research reviewed in this section relates to the study directly and concretely. They can be grouped into three general categories: (1) studies investigating structured assessments in general, (2) studies in which the CANS functional assessment is used to predict treatment decisions, and (3) studies that have identified factors in or 
predictors of ESI use. Each study is thoroughly summarized and its relevance to the current inquiry is discussed.

\section{Structured assessments.}

Assessment is an integral component of evidence-based clinical practice and is critical for case conceptualization and treatment decision making (Ponniah et al., 2011). However, few empirical studies have evaluated how assessments influence decision making in the human services (Schwalbe, 2004). Even less is known regarding the effective integration of assessment information into clinician's treatment formulation, planning, or monitoring (Mash \& Hunsely, 2005).

Limited evidence from the field of criminal justice suggests that risk assessments conducted using a structured instrument are more accurate and less biased than those that follow an unstructured format (i.e., “clinical judgments”) (Hoge, 2002). Andershed and Andershed (2016) posited that a similar outcome would be observed among social workers. More specifically, they hypothesized that assessments using a structured instrument would be perceived by experienced management-level social workers as of a higher quality than those conducted without an instrument.

Sixty social workers were provided with an identical case vignette involving a 14year old youth exhibiting conduct problems. Half of the participants used a structured instrument (ESTER) to assess the case while the other half did not employ a structured, instrument-based assessment process. Upon completion of the assessment process (structured or otherwise), the social workers were asked to identify the areas "important 
to address to prevent long-lasting psychosocial and behavioral problems" (p. 889) and to suggest concrete interventions.

After the assessments were completed, they were independently reviewed by four experienced social services managers and rated in terms of quality and potential treatment effectiveness. As hypothesized by the researchers, the social workers that used a structured assessment instrument identified a significantly greater number of risk and protective factors than did those who did not use the tool. Notably, $100 \%$ of the social workers using a structured approach identified five or more risk factors presented in the vignette while only $47 \%$ of those not using an instrument identified an equivalent number of risk factors. Likewise, the majority (67\%) of those social workers who used the structured assessment identified at least one protective factor while only two social workers $(6 \%)$ using an unstructured approach identified one or more protective factors.

Senior social worker ratings of the assessments indicated that those employing a structured approach were of a significantly higher quality, overlooked significantly fewer important factors, and were potentially more effective in informing treatment decisions. To date, this is the only published study directly comparing the content and perceived quality of assessments completed with and without structured instruments. Based on their findings the authors observed that "social workers' assessments of a youth become more evidence-based, adequate and potentially more treatment effective when a structured assessment instrument is used as compared to when it is not" (p. 897). Additionally, the authors note that their results "can and should be applied to research-based structured assessment instruments in general and not only to the instrument used in this study" (p. 897). 
Andershed and Andershed's (2016) sample was comprised of Swedish social workers that were not randomly selected, a nonequivalent structured assessment instrument, and relied on subjective judgment to determine assessment quality and effectiveness. As such, there are substantial limitations in the extent to which their findings can be generalized to the current project. However, their work provides empirical support to one of the a priori assumptions of the proposed study: structured assessments can be effective in informing treatment decisions. As Mash \& Hunsley (2005) observed in the related field of child psychology, the value of assessment is typically assumed despite very little solid supporting evidence; Andershed \& Andershed's (2016) findings, while far from conclusive, provide at least a provisional foundation from which the proposed study's rationale can be constructed.

Miller and Maloney (2013) used latent class analysis to create a compliance typology among a subsample $(\mathrm{n}=1,087)$ of frontline community corrections staff. The subsample was comprised only of participants who reported completing structured assessment tools as part of their job and using the information from these tools to make decisions or recommendations. Based on responses to an anonymous electronic questionnaire, Miller and Maloney (2013) identified three distinct classes of assessment users: (1) substantive compliers (47.7\%) - high compliance with instrument completion and moderate use of the instrument to make decisions; (2) bureaucratic compliers (39.8\%) - high compliance with instrument completion and moderately low use of the instrument to make decisions; and (3) cynical compliers (12.4\%) - high compliance with instrument completion though with admitted carelessness, minimal effort, or intentional manipulation and low use of the instrument to make decisions. 
Miller and Maloney's (2013) research focused on criminal justice practitioners and, as such, cannot be accepted as representative of other disciplines or service systems (i.e., social workers in child welfare). The precise findings cannot be generalized to the present study's sample or setting and are not of particular interest to the present context. Nevertheless, the compliance typology established by their analysis provides empirical support for another a priori assumption of the proposed inquiry: simple counts or rates of assessment completion are not sufficient to determine if the goal of assessment-driven, evidence-based treatment for children in $\mathrm{OOHC}$ is being realized.

Notably, all three of Miller and Maloney's (2013) identified classes reported high compliance with assessment instrument completion. The defining difference between groups was not whether they completed assessments but rather how well they completed them and how they used this information to inform professional decision making. Though the methodological approach is markedly different, the present study has sought to provide similar insight into the nuance of assessment tool usage beyond completion compliance.

\section{The CANS functional assessment as predictor.}

Whereas the research reviewed in the prior section pertained to structured assessment instruments in general, the body of research described at present is specific to the CANS functional assessment. More precisely, these empirical studies have investigated the degree to which responses on the CANS assessment predict various treatment-related decisions. There is a related literature regarding the CANS' use as a 
predictor for outcomes (e.g., resolution of antisocial behavior - Dunleavy \& Leon, 2011) but these are not reviewed at this time.

Anderson and Estle (2001) were the first to publish peer-reviewed research detailing the use of the CANS to identify predictors of treatment-related decisions. The researchers sought to identify factors associated with the selection of mental health service type among a sample of children $(n=150)$ being served by a rural comprehensive treatment facility. Of particular interest to the researchers were factors influencing the decision between the utilization of inpatient or community-based services for children exhibiting reactive attachment disorder symptoms.

The mental health version of the CANS assessment (CANS-MH) was used to assess the clinical status at intake of all study participants. The assessment was completed via retrospective case review. Assessed domains included mental health symptoms, risk behaviors, functioning, caregiver capacity, and strengths. Additionally, the researchers collected other relevant demographic (e.g., age, race) and clinical (e.g., maltreatment history, medication use) variables.

Using a logistic regression model, Anderson and Estle (2001) then examined which factors significantly predicted inpatient admission versus the use of communitybased care for children involved in the study. The logistic regression model resulted in a statistically significant prediction of inpatient admission with an overall prediction accuracy of $93 \%$. Significant predictors of inpatient service utilization included a history of inpatient care, a history of sexual abuse, and limited strengths identified on the CANSMH assessment. Additionally, children currently being served in an inpatient setting 
exhibited a significantly greater number of risk behaviors as indicated by the CANS than did their counterparts receiving community-based services.

Anderson and Estle's (2001) work has some substantial limitations including the use of a retrospective case review to complete the CANS functional assessment and a single site design. Additionally, the setting (i.e., exclusively rural), sample (i.e., only children exhibiting RAD symptoms), and measurement instrument (i.e., CANS-MH) do not perfectly align with the design of the study described in this proposal. Nonetheless, Anderson and Estle's (2001) early work using the CANS assessment to identify predictors of service- or treatment-related decisions established a methodological precedent with direct relevance to the proposed research.

Kisiel has used the CANS extensively in her ongoing research into complex trauma exposure among child-welfare involved youth. Two of Kisiel's (2009; 2014) studies are directly relevant to the present inquiry. Kisiel and colleagues (2009) used response patterns on the CANS assessment instrument to examine complex trauma exposure and subsequent service utilization among a large sample of children $(n=4272)$ who entered the Illinois child welfare system between July 2005 and December 2007. Like the current initiative in Kentucky, Illinois instituted an integrated assessment process centered around the CANS assessment in order to identify child and caregiver strengths and needs to inform the provision of trauma-informed treatment and services (Kisiel et al., 2009).

The researchers first used the CANS instrument to classify two groups based on trauma-exposure type: (1) children who had been exposed to complex trauma (i.e., "multiple and chronic caregiver trauma"), and (2) children who had been exposed to 
trauma that was not chronic or not caregiver-related. These groups were then compared and contrasted in relation to trauma symptoms and service utilization need. Based on CANS response patterns, more than one-third (34.5\%) of the sample met the researcher's definition of having been exposed to complex trauma. Children in the complex trauma group exhibited more trauma-related symptoms across all domains on the CANS assessment instrument. Additionally, children with chronic caregiver trauma had significantly more difficulties across functional areas (i.e., family functioning, social functioning, educational functioning) indicating a need for more intensive and extensive treatment services.

The authors note that their findings highlight the benefit of conducting a traumafocused comprehensive assessment of children entering the child welfare system in order to identify their broad range of treatment needs and match these with appropriate services. Though the researchers did not investigate the mechanism whereby these treatment and service needs are actually made, their work suggests that the CANS can be effectively used in this manner.

Kisiel later expanded on this work (Kisiel et al., 2014) to more closely examine specific patterns of trauma exposure among a large sample of child-welfare involved youth $(n=16,212)$. Following the precedent established in the earlier study, Kisiel and colleagues (2014) first used response patterns on the CANS assessment to identify distinct trauma experiences and categorize study participants into groups based on similar trauma exposure patterns (e.g., violent trauma, attachment trauma). These trauma groups were then compared in terms of symptom manifestation and severity. Interestingly, this stage of the analysis also employed the CANS assessment by mapping specific CANS 
items onto trauma symptom domains (e.g., affective and physiological dysregulation). Finally, the identified trauma groups' level of child welfare service need were compared.

Of greatest relevance to the present inquiry, Kisiel and colleagues (2014) found that youth with different trauma exposure types—as determined by CANS assessment response patterns — experienced different trajectories through the child welfare system. For example, youth who had experienced both violent and non-violent trauma were $25 \%$ more likely to experience a placement disruption than youth with other types of trauma. Likewise, youth who had been exposed to violent trauma were $37 \%$ more likely to be placed in a psychiatric hospital.

Though the researchers did not directly investigate the CANS assessment's role in informing service delivery or treatment planning (as was the objective of the present study), their use of the CANS to identify specific client groups with unique child welfare related trajectories implies that such an investigation is warranted. Perhaps even more importantly, Kisiels' $(2009 ; 2014)$ research program illustrates a novel and well-executed approach for using state gathered and maintained CANS assessment data to identify and answer compelling questions regarding a state's child welfare practice landscape.

Lardner (2015) examined the relationship between scores on the CANS assessment and level of restrictiveness decisions for a large sample $(n=5,230)$ of child welfare involved youth. He aimed to assess the amount of variation in identified level of need at the time of intake (as identified by the CANS) that could be accounted for by the placement setting.

Using the CANS assessment administered at intake, Lardner (2015) first calculated a mean domain score by summing the scores of all items in the section and 
then diving this sum by the total number of items. To aid in interpretation, this quotient was then multiplied by 10 to create a range of possible scores between 0 and 30 . These five domain scores were then summed to calculate a total CANS score. The five domains included in the analysis were (1) life domain functioning, (2) child behavioral/emotional needs, (3) child risk behaviors, (4) caregiver needs and strengths, and (5) child strengths. Basic demographic variables (gender, race, and age) were collected and used to control for intake CANS scores. Program restrictiveness was based upon a level of intensity rating applied to each child serving agency in the state; scores ranged from 5 to 15 with higher scores reflecting greater intensity.

Lardner (2015) then used a multilevel regression analysis to assess the relationship between calculated CANS scores and placement level. Initial model estimates indicated that approximately $35 \%$ of variation in total CANS scores at intake could be attributed to program assignment. When all other variables were held constant, each one-unit increase in program restrictiveness was associated with a 35.48 point increase in total CANS score.

Lardner's (2015) work is significant to the proposed study in two primary regards—one methodological and the other conceptual. Methodologically, Lardner's (2015) treatment of CANS domain scores and total score within a multilevel regression model provides an example of predictor identification and calculation that has informed the present author's analytic strategy. On a more conceptual level, Lardner (2015) has concluded that his findings support the utility of functional assessments, like the CANS, to inform treatment decision making for youth in out of home care. As such, replicating 
this line of inquiry with a focus on intervention decision making (as opposed to placement decision making) is a logical extension.

Taken together, these empirical studies clearly establish a well-tested precedent for using CANS domain scores or response patterns as predictors for modeling case-level treatment planning or decision making. While the present author is not aware of any published research evaluating CANS responses as predictors of ESI selection, their use in informing other aspects of case planning (e.g., intensity of treatment, placement restrictiveness) suggests that such an inquiry is viable.

Researchers have also explored novel approaches to using the CANS to inform treatment decision making beyond individual, case-level factors. For instance, Cordell, Snowden, and Hosier (2016) used recursive partitioning to identify particular CANS items associated with the most elevated levels of clinical need and Chor and colleagues (2012; 2013) have explored the use of the CANS assessment as part of an algorithm to automatically generate placement recommendations for child welfare administrative teams to consider. While these research programs are not as directly relevant to the present study as those reviewed in greater detail above, they further illustrate the methodological breadth with which the CANS role in treatment decision making can be examined.

\section{Predicting the use of ESIs.}

While a relatively extensive body of research literature has investigated factors associated with practitioner attitudes toward or knowledge of EBP in general (e.g., Aarons, 2004; Abrefa-Gyan, 2016; Beidas et al., 2016; Gray, Elhai, \& Schmidt, 2007; 
Nelson \& Steele, 2007), a much more limited number of empirical studies have investigated factors predicting the use of specific interventions or practices (i.e., ESIs). (Tuten et al p. 260). Similar to the present study, the empirical studies reviewed in this section used an EBP conceptual framework to investigate factors associated with or predicting the adoption of one or more specific ESIs.

Gioia \& Dziadosz (2008) conducted a mixed methods study examining a small sample $(n=14)$ of mental health practitioners as they adopted four evidence-based practices. The selected EBPs—reflecting specific practices consistent with the term ESI as used in this proposal—included integrated dual disorders treatment, cognitive therapy, dialectical behavior therapy, and McFarlane's Multi-Family Therapy. These practices were selected because they are manualized treatments with documented effectiveness for individuals with severe mental illness.

The researchers conducted a longitudinal series of semi-structured interviews to explore the participants' experiences learning and integrating the EBPs into their practice over the course of twenty-four months; in addition to the qualitative data, participants completed the Evidence-Based Practice Attitudes Scale (EBPAS) after each interview session. Consistent with the mixed methods nature of the study, the analytic strategy consisted of both thematic analysis of interview transcripts and longitudinal comparison of EBPAS scores over the course of the two-year study.

A number of conditions were identified that facilitated the adoption of the four EBPs including consistent supervision, use of outside experts, and observing positive client growth as a direct result of EBP training. Impeding conditions included a shifting practice landscape (e.g., newly introduced state regulations, changing certification 
requirements), lack of fidelity measures, and supervisor/administrator turnover. Scores on the EBPAS suggested that as practitioners developed increased competency in the adopted EBPs, their attitudes and perceptions of evidence-based practice in general became more positive.

Of key interest to the present study is the researchers' conclusion-supported with both quantitative and qualitative data - that with appropriate supervisory and administrative support, practitioners can adopt new EBPs and that this process tends to be self-reinforcing. However, the process of learning to deliver new EBPs is lengthy and implementation timeframes may underestimate the amount of time practitioners require to feel competent. This observation will be particular important to remain cognizant of when interpreting the present study's findings.

Craig and Sprang (2010) examined the self-reported trauma practices of a national sample $(n=671)$ of clinical social workers and psychologists. Study participants completed the Trauma Practices Questionnaire (TPQ). The 19 items of the TPQ were mapped onto six trauma therapies: (1) cognitive therapy, (2) behavioral therapy, (3) eye movement desensitization reprocessing, (4) eclectic therapy, (5) psychodynamic therapy, and (6) solution-focused therapy. Scores in each of these six domains served as the study's dependent variables. Additionally, scores from the first three modalities were summed to create an "evidence-based practice" variable while the cumulative scores of the last two modalities formed an aggregate "non evidence-based practice" variable.

Using stepwise regression, the researchers then sought to identify significant predictors of evidence-based practice using ten predictor variables: gender, age, disciplinary licensure, highest degree obtained, years of experience, type of work setting, 
special trauma training, percentage of caseload with PTSD, percentage of caseload below 18 , and percentage of caseload over 60 . The final regression equation indicated that specialized trauma training, older age, and percentage of PTSD on the caseload significantly predicted the use of evidence-based practices. A similar model was then tested to determine predictors of non evidence-based practice. Interestingly, specialized trauma training, older age, and percentage of PTSD on the caseload also significantly predicted the use of non evidence-based practices. Additionally, the final regression model indicated that clinical social workers were more likely to employ non evidencebased practices than clinical psychologists. It should also be noted that the non evidencebased equation accounted for less variance (6\%) than the evidence-based equation (12\%).

Craig and Sprang (2010) posit that the similar predictors for both evidence-based and non evidence-based practice may reflect a basic assumption that "working through the trauma" can be achieved in different ways. Noting that the amount of variance accounted for by both equations was small despite an adequate predictor to sample size ratio, the researchers concluded that "a number of other variables must be significant predictors of use of therapy practices beyond the demographic, work setting, and caseload characteristics explored in this study" (p. 505). This observation is significant to the current inquiry, as it represents a novel approach to the prediction of treatment selection drawing upon factors largely neglected in the current research literature.

Beidas and colleagues (2015) investigated the relative contribution of individual and organizational factors on therapist self-reported use of three treatment modalities: (1) cognitive behavioral therapy, (2) family therapy, and (3) psychodynamic therapy. As conceptualized by the researchers, CBT and family therapy represented the selection of 
an EBP while psychodynamic therapy represented the selection of a non-EBP. (It is important to note that the researcher's use of the term EBP in this study reflects specific treatments rather than a process and thus is more consistent with the term ESI as used in the present proposal.) The study's sample $(n=130)$ included therapists working in 19 mental health care agencies serving children and youth.

Individual factors included demographic characteristics (e.g., age, sex, ethnicity), attitudes (e.g., openness to new practices, Evidence-Based Practice Attitude scale), and knowledge (as measured by the Knowledge of Evidence-Based Services Questionnaire). Organizational factors included agency characteristics (e.g., program size, percentage of fee-for-service staff), culture and climate (as measured using the Organizational Social Context Measurement System), implementation climate (as measured using the Implementation Climate Scale), and implementation leadership (as measured using the Implementation Leadership Scale).

Using linear mixed-effects regression models with random intercepts due to the nesting of therapists within organizations, Beidas and colleagues (2015) analyzed the strength of the associations of the individual and organizational factors with use of each of the three modalities. Their findings indicated that organizational factors accounted for $23 \%$ of the variance in therapists' use of CBT while individual factors accounted for $16 \%$. For family therapy, organization factors explained $19 \%$ of the variance with just $7 \%$ accounted for by individual factors. By contrast, individual factors accounted for more variance $(20 \%)$ in the use of psychodynamic therapy than did organizational factors (7\%). Based on their findings, the researchers concluded that organizational factors were 
more likely to drive the use of EBPs while individual factors were more likely to drive the use of non-EBPs.

Tuten and colleagues (2016) sought to identify individual and organizational predictors of self-reported evidence-based intervention (EBI) use among a sample $(n=$ 180) of National Association of Social Workers (NASW) members. Using mail and internet surveys, the researchers asked practicing social workers if they currently used EBIs in their place of work. The researchers' definition of EBI-“"specific interventions that have been empirically tested and validated" (p. 254) — is consistent with term ESI as used in the present work. Additionally, participants completed portions of the EvidenceBased Practice Questionnaire and the Organizational Climate Measure and provided basic demographic and practice characteristics.

Using logistic regression, the researchers examined which individual or organizational factors significantly predicted current use of EBIs. The majority of respondents $(68.7 \%)$ reported using EBIs in their work. Findings indicated that more knowledgeable practitioners and those who worked in organizations that valued innovation and flexibility were significantly more likely to employ EBIs in their practice.

Although Tuten and colleagues (2016) relied only on self-reported EBI use and did not analyze specific interventions, their research was explicitly practice-oriented. As such, their work differs from much of the existing research that focuses on "the more elusive term EBP” (p. 259) and associated process-oriented activities (e.g., looking up research evidence). The researchers concede that it is impossible to know to what degree reported EBI use among study participants reflects actual EBI use and note that future 
research attention should explore actual EBI usage. Importantly, this study has focused exclusively on actual practices.

These empirical studies investigating factors associated with or significant predictors of ESI use have informed the present study at the methodological level. For example, Beidas and colleagues' (2015) multilevel investigation of ESI usage highlights the importance of accounting for nested data structures. However, perhaps even more importantly than what these studies have addressed is the substantial gap that has yet been unaddressed.

Of the empirical studies investigating the adoption of specific interventions or practices within an evidence-based framework (already a fractional subset of the broader EBP literature) only two - Craig and Sprang (2010) and Beidas et al. (2015) — have sought to identify factors that may influence the selection of specific treatment approaches or ESIs. Even so, they have done so with little consideration of the client's needs, strengths, conditions, etc. In the evidence-based medicine model developed by Haynes and colleagues (2002) and adapted for social work by Regehr and colleagues (2007), such factors are described as the clinical state and circumstances and are recognized as "key and often dominant factors in clinical decisions" (Haynes et al., 2002, p. 384). It is unfortunate that such "dominant factors" have been neglected in efforts to understand practitioner ESI selection; the intent of the present research is to help close this gap. 


\section{Summary of the concretely-relevant literature.}

As described in the next chapter, the analytic strategy of the present study involved the development of a multilevel model to investigate the relationship between response patterns on the CANS functional assessment and the prescription of empirically supported interventions. As such, it is fitting to envision the literature reviewed in this section in such terms. Even when exploratory in nature, multilevel model building involves "a wide range of theoretical assumptions" (Hox, 1995, p. 7); the first studies reviewed in this section (Andershed \& Andershed, 2013; Miller \& Maloney, 2016) provide at least some empirical support undergirding the foundational assumptions of this study's design. Specifically, they suggest that structured assessments can be used to generate high-quality treatment recommendations, that completion compliance tends to be high, but that there is variability in how accurately they are completed or how they are integrated into the decision making process.

The next set of studies (Anderson \& Estle, 2001; Chor et al., 2012; Chor et al., 2013; Cordell et al., 2016; Kisiel, 2009; Kisiel, 2014; Lardner, 2015) relate to the predictor side of the model equation. These studies illustrate diverse ways that CANS scores and response patterns have been used to predict various treatment decisions. Studies in this section have been most influential in the development of the present study's methodology and analytic plan.

Finally, the last set of studies (Beidas et al., 2015; Craig \& Sprang, 2010; Gioia \& Dziadosz, 2008; Tuten et al., 2016) reflect the dependent variable or outcome of interest. To varying degrees of specificity, these studies have investigated the factors that 
influence the use of ESIs. As important as the factors that these studies have identified as salient to ESI adoption are the client-level factors that remain unexplored. 
CHAPTER 3:

\section{RESEARCH METHODS}

\section{Research Context and Design}

Project SAFESPACE seeks to promote the use of assessment-driven, evidencebased treatment for children in out-of-home care. As part of this initiative, all children entering out of home care are administered a functional assessment. This study explores the degree to which responses on the functional assessment predict the prescribed treatment modality. The research design is a cross-sectional secondary data analysis.

\section{Sample}

The population of interest for the proposed study is children in out of home care who have been administered a standardized functional assessment. The sampling frame consists of all children who (1) entered out of home care after Project SAFESPACE implementation in their county of origin, (2) screened in and were administered a functional assessment and for whom complete assessment data is available, and (3) were five years of age or older on the date of their initial functional assessment.

\section{Instrumentation}

The secondary data used in this study originates from two practitioner-completed forms: (1) the Child and Adolescent Needs and Strengths (CANS) functional assessment, and (2) the CANS assessment report. Both forms are completed digitally on an internet- 
based information system hosted by a contracted third party. Data are then transmitted to the statewide automated child welfare information system (SACWIS). Only the initial assessment for each case is included in the analysis.

\section{Child and Adolescent Needs and Strengths assessment.}

The CANS is a "multiple purpose information integration tool" designed to be the output of an assessment process (Praed Foundation, 2016). Developed using a communimetric (as opposed to a psychometric) approach, the CANS is interpretable at the item level, with each individual instrument item having direct implications for differential action (Lyons, 2009). In other words, every item in the instrument serves "to inform choices among possible interventions or approaches" by not only identifying the presence/absence of a concern but also describing the level of action required in a directly interpretable manner (Lyons, 2009, p. 31).

The CANS is widely used throughout the United States across a broad range of service settings, including extensive use in child welfare systems (Rosanbalm et al., 2016). Service systems adopting the CANS instrument frequently develop versions tailored to fit their specific informational and cultural needs (Praed Foundation, 2015). Two versions of the CANS assessment have been developed for use with child welfare populations in Kentucky—one intended for use with children younger than five years of age and one for use with children five years old or older. The present study will use the latter version only (KY CANS 5+).

Only individuals who have been trained and certified can complete the KY CANS 5+. Training consists of approximately seven hours of face-to-face instruction followed 
by a series of online video modules (L. Minton, personal communication, September 15, 2017). Upon completion of the training, the individual must take a certification exam wherein he or she completes a CANS assessment using a case vignette. A reliability of .70 or higher is required for successful completion at the practitioner level while trainers must score above an .80 (D. Hickerson, personal communication, September 15, 2017).

The KY CANS 5+ consists of six primary domains: (1) life domain functioning, (2) acculturation, (3) child strengths, (4) child emotional/behavioral needs, (5) child risk behaviors, and (6) caregiver needs/strengths. Responses to specific items within these domains may trigger additional modules (e.g., trauma module). Domains and modules consist of a varying number of individual items all of which are rated from zero to three. Individual items rated with a two or a three are considered "actionable" and thus must be addressed in the child's service or treatment plan (Praed Foundation, 2016). In addition to individual item ratings, dimension scores can be calculated by summing items within each domain (Praed Foundation, 2016).

A large body of research has investigated the measurement properties of the CANS. Anderson, Lyons, Giles, Price, and Estle (2003) obtained an inter-rater reliability of 0.85 between researchers conducting a retrospective case reviews and of 0.81 between researchers' case review assessments and those completed prospectively by caseworkers at the time of client admission. More recently, Anderson (2008) reported an inter-rater reliability of .80 between a certified CANS trainer and three trained data collectors. These reported findings are generally consistent with the Praed Foundation's (2016) claim that the average reliability of the CANS is 0.75 with vignettes, 0.84 with case records, and 0.90 with live cases. 
The CANS has demonstrated an adequate degree of concurrent validity with the Child and Adolescent Functional Assessment Scale (CAFAS; Dilley, Weiner, Lyons, \& Martinovich, 2007). Studies conducting factor analyses using the CANS have produced a similar three-factor solution (caregiver problems, internalizing behaviors, externalizing behaviors) suggesting a consistent underlying structure and some degree of construct validity (Rosanbalm et al., 2016). As discussed in greater depth in the literature review section, the predictive validity of the CANS has also been supported by a number of peerreviewed studies (e.g., Kisiel et al., 2009; Yampolskaya, Armstrong, \& Vargo, 2007).

\section{CANS assessment report.}

Upon completion of the actual CANS instrument, a CANS assessment report is generated. Actionable items from the CANS instrument (i.e., items rated 2 or 3 ) are automatically populated on this digital document providing a summary of the most salient clinical concerns. If applicable, the report also indicates if the items have improved, worsened, or remained stable since the previous assessment.

Using this information (at least in theory), the clinician then identifies the primary focus of treatment and prescribes one or more treatment modalities (i.e., ESIs). The CANS assessment report offers 18 ESI options: (1) adolescent community reinforcement, (2) brief strategic family therapy, (3) child-parent psychotherapy, (4) cognitive therapy, (5) cognitive behavioral therapy, (6) dialectical behavior therapy, (7) eye movement desensitization and reprocessing, (8) family therapy, (9) parent-child interaction therapy, (10) prolonged exposure therapy, (11) restorative approach, (12) sanctuary model, (13) seven challenges, (14) structural family therapy, (15) trauma recovery and empowerment 
model, (16) trauma-focused cognitive behavioral therapy, (17) wraparound, and (18) other. Working collaboratively, Project SAFESPACE representatives selected these modalities based on the following considerations: (a) focused on child welfare and/or trauma, (b) inclusive of practices already in use by providers, and (c) cognizant of sustainability concerns (e.g., availability and cost of training opportunities) (B. Jordan,

personal communication, January 17, 2018). Clinicians who select the "other" option are prompted to input the name of the prescribed intervention or treatment.

After selecting the appropriate ESIs, the practitioner selects the intensity at which each modality will be delivered (e.g., more than once a week). The CANS assessment report will also serve as the source document for important demographic data including the child's age, gender, and race; the name of the clinician completing the assessment; and the agency providing behavioral health services.

\section{Research Variables}

\section{Dependent variables: ESI selection.}

The outcome of primary interest in the present study is the treatment modality prescribed by the clinician after completing the CANS and reviewing the CANS assessment report. Each of the 18 possible ESIs will be coded as a unique dichotomous variable $(0=$ not prescribed $/ 1=$ prescribed $)$. Such a coding scheme is necessary to permit the analysis of cases in which multiple modalities are identified. Treatment intensity will not be a focus of the present analysis.

A challenge inherent in this data is substantial variation of specificity and scope within the set of potential ESI recommendations as listed on the CANS Assessment 
Report. For example, parent-child interaction therapy (PCIT) is a manualized intervention with concrete training and certification requirements (www.pcit.org) whereas family therapy is a term that may be applied to any psychotherapeutic approach that involves a family unit. Additionally, many of the modalities within this list are nested within a vertical hierarchy. For example, the more specialized modalities of dialectical behavior therapy (DBT) and cognitive therapy (CT) are both subsets of the broader cognitivebehavioral therapy (CBT) family of interventions.

This challenge can be mitigated, at least in part, by expanding the focus of inquiry to broader treatment categories. To accommodate this, a second set of four dichotomous variables $(0=$ not prescribed $/ 1=$ prescribed $)$ will be computed from the original individual ESI variables: (1) trauma-focused treatment, (2) behavioral-focused treatment, (3) family-focused treatment, and (4) substance use-focused treatment. While individual ESI prescriptions will still be analyzed at a descriptive-level of analysis (e.g., counts), the composite focus-of-treatment variables will serve as the dependent variables for more advanced modeling. The process and rationale for identifying the proper alignment of individual ESIs to categorical groupings is described below.

Information regarding the target population and focus of treatment for each of the potential ESI selections was obtained from the California Evidence-Based Clearinghouse for Child Welfare (CEBC; www.cebc4cw.org). The CEBC was selected because (1) it is open access, (2) it summarizes peer-reviewed outcome studies in a concise format, and (3) it focuses exclusively on child welfare. As the present study focuses on the EBP decision making of practitioners, it was deemed important to draw ESI characteristics from a resource that is conducive to a typical practice environment (see Thyer, Babcock, 
and Tutweiler, 2017). Only interventions that had received a CEBC scientific rating of promising research evidence (i.e., at least one study utilizing some form of control), supported by research evidence (i.e., at least one rigorous randomized controlled trial), or well supported by research evidence (i.e., multiple RCTs and follow-up) were included. Though listed in the CEBC database, the Restorative Approach and the Sanctuary Model were not included in any of the focus-of-treatment composite variables. The Restorative Approach is targeted toward staff members (Child Welfare League of America, 2008) and the Sanctuary Model focuses on organizational-level change (Bloom, 2013). As such, their prescription is assumed to be primarily a function of a child's placement setting rather than his or her individual assessment responses.

Twelve of the ESI options listed on the CANS assessment report will be included in the focus-of-treatment composite variables. Table 3.1 identifies the composite variable to which ESI is assigned as well as the CEBC scientific rating and child welfare system relevancy rating. Note that some ESIs may be included in more than one focus-oftreatment variable as determined by their target population and goals of treatment. 
Table 3.1. ESIs \& Focus-of-Treatment Composite Variable Inclusion

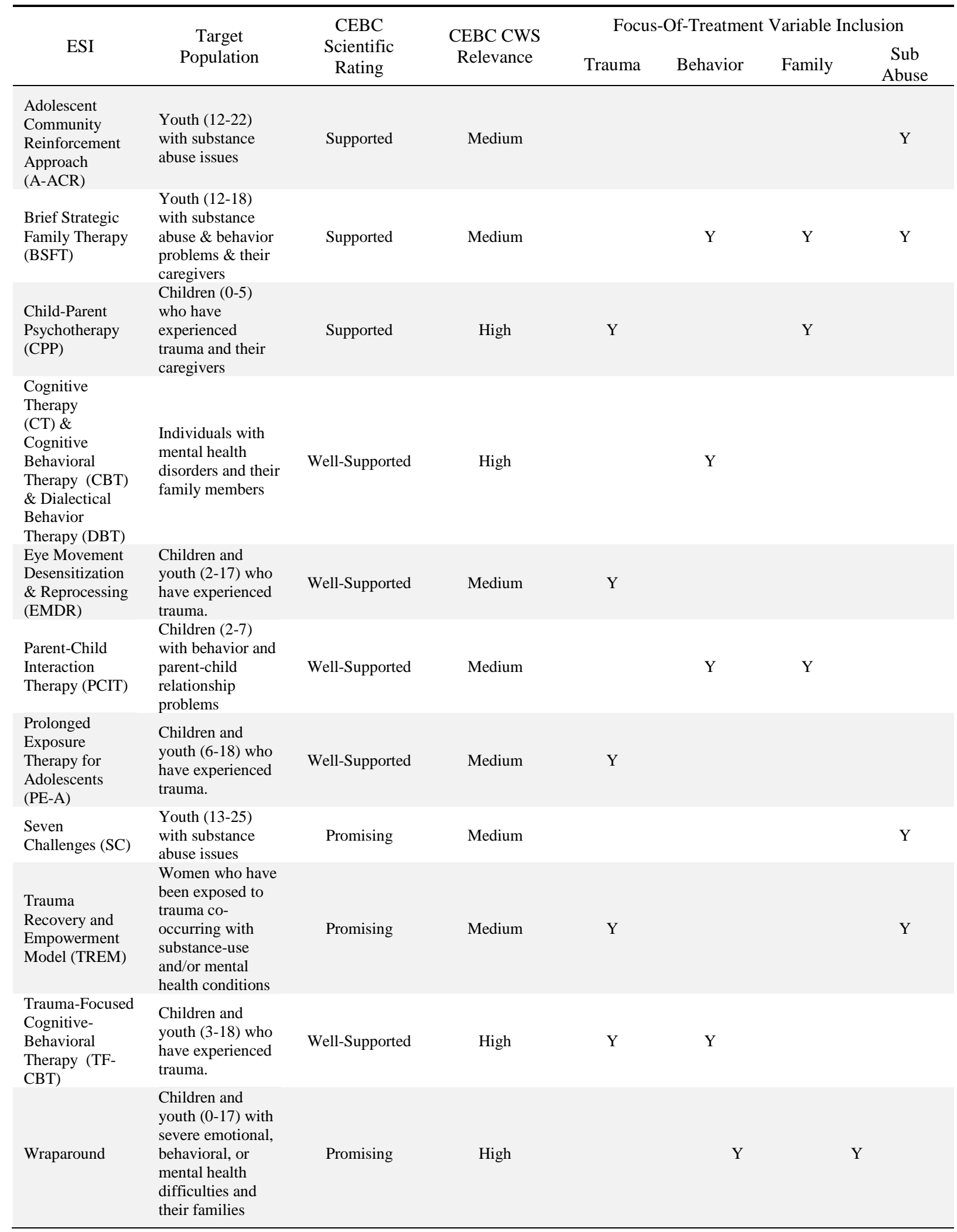




\section{Predictor variables: Assessment-identified needs.}

To examine the relationship between response patterns on the CANS functional assessment and ESI prescription, four dimension scores will be calculated —each of which is conceptually associated with one of the focus-of-treatment composite variables. Using dimension scores calculated from specific CANS domain items is recognized as a valid interpretive method by the CANS developers (Praed Foundation, 2016) and has been a methodological technique employed in prior research using the CANS (e.g., Lardner, 2015; Kisiel, 2014). Table 3.2 presents the dimension name, its source domain or module in the KY CANS 5+, the number of items comprising the dimension, and the possible value range for the calculated dimension score. As indicated by these ranges, all predictor variables will be treated as scalar measurements.

Table 3.2. Predictor Variables and Associated CANS Domain Items

\begin{tabular}{llccc}
\hline \multicolumn{1}{c}{ Dimension } & \multicolumn{1}{c}{ KYCANS5+ Source Domain } & $\begin{array}{c}\text { Number of } \\
\text { Items }\end{array}$ & $\begin{array}{c}\text { Possible } \\
\text { Values }\end{array}$ & $\begin{array}{c}\text { Cronbach's } \\
\alpha\end{array}$ \\
\hline $\begin{array}{l}\text { Trauma } \\
\text { Exposure }\end{array}$ & $\begin{array}{l}\text { Trauma Experiences Module \& Adjustment Sub } \\
\text { Module (excluding time before treatment item) }\end{array}$ & 17 & $0-51$ & .86 \\
$\begin{array}{l}\text { Emotional / } \\
\text { Behavioral } \\
\text { Problems }\end{array}$ & $\begin{array}{l}\text { Child Emotional/Behavioral Needs Domain } \\
\text { (excluding trauma \& substance use items) \& } \\
\text { Child Risk Behaviors Domain }\end{array}$ & 24 & $0-72$ & .84 \\
$\begin{array}{l}\text { Family / } \\
\text { Caregiver } \\
\text { Functioning }\end{array}$ & $\begin{array}{l}\text { Life Functioning Domain (first two items only) \& } \\
\text { Child Strengths (first two items only) \& }\end{array}$ & 24 & $0-72$ & .93 \\
$\begin{array}{l}\text { Caregiver Needs/Strengths } \\
\text { Usbstance }\end{array}$ & $\begin{array}{l}\text { Child Emotional/Behavioral Needs Substance } \\
\text { Use Item \& Substance Use Module }\end{array}$ & 7 & $0-21$ & .94 \\
\hline
\end{tabular}




\section{Analytic Strategy}

Prior to testing the study's research hypotheses, an extensive descriptive analysis of the data was conducted. This descriptive information provided insight into the data's structure and helped identify contextual factors that informed the subsequent model building process(e.g., sample characteristics, potentially salient covariates). Moreover, as the universal trauma screening and assessment initiated by Project SAFESPACE represents a substantial change to the child welfare and behavioral health practice environment, descriptive information about the service landscape immediately postintervention is valuable not only from a research perspective but also for practitioners, administrators, and policymakers. A number of secondary research questions were explored in this initial analytic phase including:

1. Which ESIs are most frequently prescribed by clinicians completing the CANS functional assessment? Which ESIs are most frequently prescribed at an agency level?

Conducting a frequency distribution of the prescribed ESIs will identify those treatment modalities that are the most/least frequently selected for treatment in the practice environment. The counts of ESIs prescribed can also provide insight regarding the approach to therapeutic treatment (i.e., eclectic vs. homogenous) by case, clinician, or agency. As the CANS assessment report permits the identification of an "other" option when selecting a treatment modality, this analysis will determine if additional ESIs need to be considered for addition to the composite focus-of-treatment variables. 
2. What do CANS domain scores and item responses indicate about the treatment needs of the Project SAFESPACE population?

Because of its roots in communimetric theory, the CANS is not intended for norm-based decision making and normative data are not available (Rosanbalm et al., 2016). However, a closer examination of CANS responses can provide valuable descriptive information about the study's sample and the Project SAFESPACE population. For example, using responses to selected CANS items one can gauge the prevalence of maltreatment experiences or describe particular constellations of trauma exposure (see Kisiel, 2014 for a methodologically sophisticated example of this approach). For the present study, descriptive statistics related to the dimensions of interest (i.e., predictor variables) will provide context for model interpretation.

3. Do assessed clinical needs vary by child demographic (age, gender, or race) variables?

Mean scores across the four predictor variable scales will be examined by age, gender, and race. This information will be used to inform the inclusion of factors or covariates during the model building stage to control for extraneous variability. Additionally, these findings may illuminate differential experiences for particular population subgroups (e.g., minority children, young children) that warrant additional exploration in future research projects. 
Building upon these initial analyses, the primary line of inquiry was explored: Is there a predictable relationship between particular CANS functional assessment response patterns and the type of ESI prescribed? If so, how much variance in treatment prescription is accounted for by associated CANS assessment items? Implicit to this inquiry are four testable hypotheses:

$\mathrm{H}_{1}$ : Trauma-related CANS functional assessment items will predict the prescription of a trauma-focused ESI.

$\mathrm{H}_{2}$ : Emotional/behavioral-related CANS functional assessment items will predict the prescription of a behavior-focused ESI,

$\mathrm{H}_{3}$ : $\quad$ Family functioning-related CANS functional assessment items will predict the prescription of a family-focused ESI.

$\mathrm{H}_{4}$ : Substance use-related CANS functional assessment items will predict the prescription of a substance abuse-focused ESI.

\section{Multilevel modeling.}

Regression models are tools for predicting and/or describing the relationship between variables (Kreft \& DeLeeuw, 1998) and, as such, are an appropriate analytic technique for exploring the research question and hypotheses noted above. However, if regression is used to analyze hierarchically structured data without accounting for 
different levels, problems of both statistical assumption (e.g., independence of errors) and practical interpretation (e.g., ecological fallacy) arise (Meyers, Gamst, \& Guarino, 2013; Tabachnick \& Fidell, 2013). Data are hierarchically structured when smaller units of analysis (e.g., students) are contained, or "nested" within larger grouping units (e.g., classrooms) (Robson \& Pevalin, 2016); this nesting may occur at two or more levels. Multilevel modeling (MLM) is specialized type of regression model that accommodates for hierarchically structured data by permitting intercepts and slopes to vary among higher level units (Tabachnick \& Fidell, 2013).

The hypothesized model consists of two levels: (1) clients nested within (2) clinicians. Table 3.3 summarizes the model data components including their structural level, values, and level of measurement.

Table 3.3. Model Data Components

\begin{tabular}{|c|c|c|c|c|}
\hline Variable & Level & Description & Values & Measurement \\
\hline Trauma Exposure & Level 1 - Client & Predictor (Covariate) & $0-51$ & Scale \\
\hline $\begin{array}{l}\text { Behavioral } \\
\text { Problems }\end{array}$ & Level 1 - Client & Predictor (Covariate) & $0-72$ & Scale \\
\hline Family Functioning & Level 1 - Client & Predictor (Covariate) & $0-72$ & Scale \\
\hline Substance Abuse & Level 1 - Client & Predictor (Covariate) & $0-21$ & Scale \\
\hline Age & Level 1 - Client & Potential Predictor (Covariate) & $5-19$ & Scale \\
\hline Gender & Level 1 - Client & Potential Predictor (Factor) & $\begin{array}{c}0=\text { male } \\
1=\text { female }\end{array}$ & Nominal \\
\hline $\begin{array}{l}\text { Trauma-Focused } \\
\text { Treatment }\end{array}$ & Level 1 -Client & DV - Outcome Variable 1 & $\begin{array}{c}0=\text { not prescribed } \\
1=\text { prescribed }\end{array}$ & Nominal \\
\hline $\begin{array}{l}\text { Behavior-Focused } \\
\text { Treatment }\end{array}$ & Level 1 - Client & DV - Outcome Variable 2 & $\begin{array}{c}0=\text { not prescribed } \\
1=\text { prescribed }\end{array}$ & Nominal \\
\hline $\begin{array}{l}\text { Family-Focused } \\
\text { Treatment }\end{array}$ & Level 1 - Client & DV - Outcome Variable 3 & $\begin{array}{c}0=\text { not prescribed } \\
1=\text { prescribed }\end{array}$ & Nominal \\
\hline $\begin{array}{l}\text { Substance Abuse- } \\
\text { Focused Treatment }\end{array}$ & Level 1 - Client & DV - Outcome Variable 4 & $\begin{array}{c}0=\text { not prescribed } \\
1=\text { prescribed }\end{array}$ & Nominal \\
\hline Clinician & Level 2 - Clinician & Level 2 Grouping Unit & $1-381$ & Nominal \\
\hline
\end{tabular}


Among the numerous techniques for and approaches to multilevel analysis, models examining categorical data are "relatively recent additions to the methodological toolbox" (Powers, 2012, p. 57). These nonnormally distributed outcome variables are modeled using generalized linear models (GLM). The GLM consists of three fundamental components: (1) an outcome variable $Y$ with a specific error distribution (i.e., random distribution) that has mean $\mu_{i}$ and variance $\sigma^{2}$; (2) a link function that connects the expected values $(\mu)$ of $Y$ to the transformed predicted values for $\eta$, and (3) a linear structural model that produces a latent predictor $\eta$ of the outcome variable $Y$ (Heck et al., 2012; Hox et al., 2018).

Because the dependent variables in the present analysis are dichotomous, the probability distribution is binomial $(\mu, n)$ with $n$ representing the number of trials (Hox et al., 2018). The mean $(\mu)$ can be interpreted as the probability of success $(\pi)$ (i.e., the event coded 1) (Heck et al., 2012). With only one trial $(n=1)$ there are only two possible outcomes -0 or 1 . This scenario is a special case of the binomial distribution called the Bernoulli distribution (Hox et al., 2018).

The logit link function is the canonical link function for the binomial distribution. The logit link function assumes a binary outcome variable $(Y)$ with probability $\pi$. The probability $\pi$ for individual $i$ is then transformed into the logit:

$$
\eta_{i}=\log \left(\frac{\pi_{i}}{1-\pi_{i}}\right)
$$

Thus, the logit coefficient $(\eta)$ represents the $\log$ odds of $Y=1$ as opposed to $Y=0$. 


\section{Sample size.}

The estimation methods employed in multilevel analysis are asymptotic and thus assume a large sample size (Hox et al., 2018). The most cited sample size guideline for linear multilevel models calls for at least 30 first-level units nested within 10 or more second-level clusters if the interest is on fixed effects; at least 50 second-level clusters are recommended if random effects are of interest (Schoeneberger, 2016). However, the analysis of binomial data (i.e., multilevel logistic models) requires larger sample sizes than multilevel linear models (Hox et al., 2018). In the case of these generalized multilevel models, a general 50/50 rule has been suggested: 50 groups with a membership of 50 first-level units (Moineddin, Matheson, \& Glazier, 2007).

Preliminary estimates of the available data suggested an adequate number of level-two grouping units (i.e., clinicians), though most of these groups were expected to be comprised of fewer than 50 level-one units (i.e., cases). Receipt of the data confirmed ample level-two grouping units (381) with an average group membership below the recommended level $(\sim 7)$. This data structure was deemed adequate for the proposed multilevel logistic analysis. While the second-level clusters ranged in size from 1 to 64; unequal sample sizes among each level are expected and pose no computational problems (Tabachnick \& Fidell, 2013). Moreover, smaller than recommended group sizesparticularly when the number of level-two clusters is great and the model is not complex - will still result in adequate statistical power and produce robust fixed effect estimates. In such cases, it is primarily the group-level random effect variances that may be overestimated (Schoeneberger, 2016). Importantly, only first-level fixed effects were of interpretive interest in the present analysis. 


\section{Variable centering.}

Predictor variables are often centered prior to conducting MLM. Centering entails subtracting the mean from each score (Meyers et al., 2013); in effect centering transforms raw scores into deviation scores. The two most common centering techniques are grand mean centering - each score is centered against the mean of the entire sample — and group mean centering - scores are centered against the mean of other cases in their group or cluster.

Centering predictor variables often aids in the interpretation of the fixed intercept. Prior to centering, the intercept is the value of the dependent variable when all predictor variables are zero (Tabachnick \& Fidell, 2013). This makes interpretation difficult when the value of zero is not meaningful. By centering the predictors, the intercept becomes the value of the DV when the predictors are equal to the mean of the sample (grand mean centering) or the group/cluster (group mean centering).

Decisions regarding the centering strategy should always reflect the research questions (Sommet \& Morselli, 2017). In the present analysis, the approach that was most conducive to intercept interpretation was to not center the predictor variables (i.e., maintain raw scores). This is because the predictor variables-composites of assessment items - $d o$ have a meaningful zero values and, in fact, the zero values represent the most readily interpretable reference point. For example, in the model examining the prescription of a trauma-focused treatment, the intercept represents the probability of being assigned a trauma-focused treatment when the assessment reflects no indication of trauma-, behavior-, family-, or substance use-related problems (i.e., all relevant items are rated as zero on the CANS assessment). 


\section{Model estimation.}

Multilevel modeling typically employs maximum likelihood (ML) estimation techniques (Tabachnick \& Fidell, 2013). The ML estimation method "produces estimates for the population parameters that maximize the probability (produce the 'maximum likelihood') of observing the data that are actually observed" (Hox et al., 2018, p. 27). For MLM with categorical outcomes, ML estimation seeks to produce the maximum quasi-likelihood - an approach that approximates the nonlinear link by a nearly linear transformation (Heck et al., 2012, Hox et al., 2018). The most efficient ML estimation methods for categorical outcomes use the Newton-Raphson and Fisher scoring methods (Heck et al., 2012). The Newton-Raphson technique is the default estimation method for generalized linear models in SPSS and was the ML estimation method used in the following analyses.

\section{Model comparison and evaluation.}

Maximum likelihood estimation techniques produce a model deviance statistic. The model deviance statistic is equivalent to $-2 * \log$ likelihood (2LL), where log is the natural logarithm and likelihood is the value of the likelihood function at the point of convergence (Heck et al., 2012). This deviance statistic can be used as an indicator of model fit; lower deviance suggests better fit than higher deviance. A number of tests and indices derived from the deviance statistic are used to compare competing models, including the $\chi^{2}$ likelihood ratio test $\left[\chi^{2}=\left(-2 * \log\right.\right.$ likelihood $\left.{ }_{\text {simpler model }}\right)-(-2 * \log$

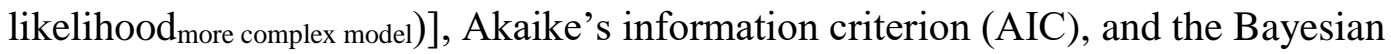
information criterion (BIC) (Heck et al., 2012, Tabachnick \& Fidell, 2013). 
However, because multilevel models with dichotomous outcomes employ an estimation procedure that maximizes an approximate likelihood function (i.e., quasilikelihood) rather than a precise likelihood, tests and indices relying on the deviance statistic are not accurate (Hox et al., 2018). The SPSS GENLIN output does provide an estimate of the $-2 \log$ pseudo likelihood for model comparison but warns: "When comparing models using pseudo likelihood values, caution should be used because different data transformations may be used across models" (IBM SPSS v25, 2018). Given the inaccuracy of the deviance statistic across substantially different models, procedural guidelines for multilevel logistic regression modeling often do not include a fit comparison between the unconditional (null) model and subsequent iterations involving level-one predictors (e.g., Heck et al., 2012; Sommet \& Morselli, 2017) although some do advocate for a fit comparison between models with identical predictors but differing random effects (e.g., Sommet \& Morselli, 2017). In fact, some authors have even suggested that "substantive sensibility" — as opposed to statistical examination—should be the analyst's primary concern when evaluating multilevel models with categorical outcomes (Heck et al., 2012, p. 162).

Given the impossibility of reliable interpretation, the deviance statistic, and any indices or tests derived from it, were not employed for model comparison in the present analysis. On the other hand, the lack of theoretical or analytical precedent for this inquiry demands caution in relying solely on substantive evaluation. As such, two empirical measures not derived from calculated model deviance were used in model comparison. The first evaluative strategy involved the examination of the model's classification 
accuracy while the second involved the calculation of a pseudo- $\mathrm{R}^{2}$ and the comparison of explained variance across models.

To compare classification accuracy across models, predicted and observed outcome group membership (i.e., prescribed / not prescribed) were cross-tabulated after each subsequent model iteration and the phi coefficient calculated. This provides the ability to (1) examine the significance and strength of the correlation between predicted and observed outcome classification and (2) assess the impact of introduced predictors on this correlation.

In multiple regression, the squared multiple correlation $\left(\mathrm{R}^{2}\right)$ can be interpreted as the "proportion of variance in the DV that is predictable from the best linear combination of the IVs" (Tabachnick \& Fidell, 2013, p. 130). When multiple levels are involved, the concept of "explained variance" becomes more complex. Hox and colleagues (2018) have outlined an approach that entails examining the proportion of explained variance by examining the residual error variances at each level in a sequence of models. However, this approach is less straightforward for multilevel binary logistic regression models because (1) most analogues to the squared multiple correlation in logistic regression are calculated using the deviance statistic of the null model and a comparison model, and (2) the underlying latent variable is rescaled to the same standard distribution $\left(\pi^{2}-3\right)$ for each model so the lowest-level residual variance is always $~ 3.29$ (Hox et al., 2018).

To overcome the first obstacle. McKelvey and Zavoina's (1975) method was employed to estimate the explained variance associated with the latent outcome $(\eta)$ using the formula:

$$
R_{M Z}^{2}=\frac{\sigma_{F}^{2}}{\sigma_{F}^{2}+\sigma_{u 0}^{2}+\sigma_{R}^{2}}
$$


In effect, the variance of $\eta$ is segmented into the lowest level residual variance $\left(\sigma_{R}^{2}\right)$, the second-level intercept variance $\left(\sigma_{u 0}^{2}\right)$, and the variance associated with the linear predictor in the fixed portion of the model $\left(\sigma_{F}^{2}\right)$. The variances of the four linear predictors were calculated in the raw data file from the modeled regression equation. The systematic variance can then be divided by the total variance to estimate the proportion of variance attributable to the linear predictor.

The second obstacle — the rescaling of the underlying latent variable — renders it useless to compare the explained first-level variance across models as the variance will always be $\sim 3.29$. However, using the total variance of the latent variable (the denominator of the McKelvey and Zavoina $\mathrm{R}^{2}$ ), a scale correction factor can be calculated using the formulas $\sigma_{o}^{2} / \sigma_{m}^{2}$ for the variance components and $\sigma_{0} / \sigma_{m}$ for the regression coefficients. Having been rescaled, the explained first-level variance can then be compared across subsequent model iterations (Hox et al., 2018).

\section{Model building process.}

The sequence of steps described below (adapted from Gamst et al., 2013; Heck et al., 2012; Tabachnick \& Fidell, 2013) summarizes the model-building process for predicting a trauma-focused treatment prescription; other outcome variables followed the same modeling procedure. All analyses were conducted in IBM SPSS Version 25 using the generalized linear mixed modeling (GLMM) procedure.

1. Data screening, cleaning, and assumption checking.

2. Creation of an "unconditional" or null model to examine the intraclass correlation (ICC). 
3. Estimation of a model with level-one, CANS-derived predictors fixed.

4. Estimation of models in which the slope for each level-one, CANS-derived predictor is permitted to vary randomly across level two units.

5. Estimation of a model with level-one, CANS-derived and demographic predictors fixed.

6. Model interpretation.

The unconditional model (Model 0).

The initial phase of the exploratory modeling process entails the fitting of an unconditional—or intercept-only—model. The unconditional model for client $i$ of clinician $j$ can be expressed as:

$$
\eta_{i j}=\log \left(\frac{\pi_{i j}}{1-\pi_{i j}}\right)=\beta_{0 j}
$$

where $\beta_{0 j}$ is the intercept for the $j$ th clinician. At the second level, the intercepts $\left(\beta_{0 j}\right)$ vary across clinicians:

$$
\beta_{0 j}=\gamma_{00}+u_{0 j}
$$

where $\gamma_{00}$ is the clinician-level intercept and $u_{0 j}$ is the random parameter capturing clinician-level variability. Through algebraic substitution, the single-equation unconditional two-level model can be expressed as:

$$
\eta_{i j}=\gamma_{00}+u_{0 j}
$$

As this equation indicates, the unconditional model estimates one fixed effect - the clinician-level intercept — and one random effect - the clinician-level variance for the intercept. 
The unconditional model permits the calculation of the intraclass correlation coefficient (ICC). The ICC $(\rho)$ is a ratio of the between-group variance to the withingroup variance at the second level (i.e., clinician):

$$
\rho=\frac{\sigma_{\text {between }}^{2}}{\sigma_{\text {between }}^{2}+\sigma_{\text {within }}^{2}}
$$

The ICC indicates the proportion of the total variance that is explained by the nested structure of the data; it can also be interpreted as "the expected correlation between two randomly drawn units that are in the same group" (Hox, Moerbeek, \& Schoot, 2018, p. 13). Higher ICC values indicate that the assumption of independence of errors is violated (Tabachnick \& Fidell, 2013) and, thus, that MLM is an appropriate analytic strategy.

Guidelines regarding the precise ICC value that can be regarded as indicative of violating the assumption of independence of errors are rare in the statistical literature. For example, Meyers and colleagues (2013) only go so far as to note that "to the extent that the ICC is greater than zero, membership in a Level 2 unit contributes to prediction and thus indicates that the independence assumption is violated" (p. 470). Likewise, Hox and colleagues (2018) provide no concrete values on the matter apart from noting in one example that an ICC of 0.10 is "rather low" (p. 5). Tabachnick and Fidell (2013) suggest that if the ICC is "trivial" single-level analysis may appropriate but do not offer a more precise definition; they do note that a practical strategy "when the need for hierarchical analysis is ambiguous" is to conduct both a single-level and a multilevel analysis and see if the results differ substantively (p. 826).

Simulation studies have shown that even relatively small ICC values can increase the probability of Type 1 errors depending on the size of the level-two grouping units. For example, Barcikowski (1981) notes that with an ICC of only 0.05 and a group size of 
10 a nominal $\alpha$ level of 0.05 is, in fact, closer to 0.11 ; with a group size of 100 , the actual $\alpha$ level balloons to 0.43 . Perhaps this is why Hox and colleagues (2018) observed the assumption of independence is "almost always" violated with nested data (p. 4). To reduce the likelihood of type 1 errors, and to adopt a conservative approach to ICC interpretation, it was determined a priori that any ICC greater than 0.05 (i.e., $5 \%$ of total variance is attributable to the data's nested structure) would be considered grounds for multilevel analysis.

\section{The fixed CANS-derived predictors model (Model 1).}

As the ICC calculated from the unconditional model indicated sufficient clinicianlevel variability in the likelihood for a client to be prescribed a trauma-focused treatment to warrant further multilevel analysis, the next phase in the model building process entailed the addition of all CANS-derived predictors as fixed effects. As there are four predictors $(X)$ of interest in the present analysis - trauma experiences, behavioral/emotional problems, family functioning, and substance abuse - the fixed predictor level-one model can be expressed as:

$$
\begin{gathered}
\eta_{i j k}=\log \left(\frac{\pi_{i j}}{1-\pi_{i j}}\right) \beta_{0 j}+\beta_{1} X_{1 i j}+\beta_{2} X_{2 i j}+\beta_{3} X_{3 i j}+\beta_{4} X_{4 i j} \\
\text { or } \\
\eta_{i j k}=\log \left(\frac{\pi_{i j}}{1-\pi_{i j}}\right) \beta_{0 j}+\beta_{1} \text { trauma }_{i j}+\beta_{2} \text { behavior }_{i j}+\beta_{3} \text { family }_{i j} \\
+\beta_{4} \text { substance }_{i j}
\end{gathered}
$$

where $\beta_{0 j}$ is the intercept for the $j$ th clinician. As with the unconditional model, the intercepts vary across clinicians: 


$$
\beta_{0 j}=\gamma_{00}+u_{0 j}
$$

However, the level-one within-group slopes for the four predictor variables are fixed with no corresponding random components. In other words, the slope is not free to vary across clinicians:

$$
\begin{aligned}
& \beta_{1 j k}=\gamma_{10} \\
& \beta_{2 j k}=\gamma_{20} \\
& \beta_{3 j k}=\gamma_{30} \\
& \beta_{4 j k}=\gamma_{40}
\end{aligned}
$$

Through substitution, the two-level fixed predictor model can be expressed as the single equation:

$$
\eta_{i j}=\gamma_{00}+\gamma_{10} \text { trauma }_{i j}+\gamma_{20} \text { behavior }_{i j}+\gamma_{30} \text { family }_{i j}+\gamma_{40} \text { substance }_{i j}+u_{0 j}
$$

As indicated, the fixed predictor model has five fixed effects (the intercept and four slope coefficients) and one random effect capturing clinician-level variation $\left(u_{0 j}\right)$.

In this step the contribution of each level-one, CANS-derived predictor was assessed. The model produced in this step was compared with the model from step one to see if the inclusion of the level-one predictors improved the model.

The CANS-derived predictors models with random slopes (Model 2).

Whereas level-one predictors were fixed in the previous step, in this series of models the level-one predictors were permitted to be random one at a time. Of analytic interest was the presence of any significant random effects of the CANS-derived predictors, which would indicate that the relationship between CANS responses patterns and the prescription of a trauma-focused ESI varies across clinicians. 
This step involved the systematic analysis of 16 different models. In contrast to the fixed predictor model, the within-cluster slope was defined as randomly varying across clinicians:

$$
\beta_{1 j k}=\gamma_{10}+u_{1 j}
$$

where $\gamma_{10}$ is the clinician-level mean intercept coefficient for the first predictor (trauma experiences) and $u_{1 j}$ is a random parameter representing the difference in individual clinician coefficients from this mean. This results in the combined equation:

$$
\begin{gathered}
\eta_{i j}=\gamma_{00}+\gamma_{10} \text { trauma }_{i j}+\gamma_{20} \text { behavior }_{i j}+\gamma_{30} \text { family }_{i j}+\gamma_{40} \text { substance }_{i j} \\
+u_{1 j} \text { trauma }+u_{0 j}
\end{gathered}
$$

The fixed CANS-derived and demographic predictors model (Model 3).

In the final phase of the model building process, child-level demographic predictors were included with the four CANS-derived predictors. One of the demographic predictors — gender — was dichotomously coded with males as the reference group. The full equation for model three can be expressed as:

$$
\begin{gathered}
\eta_{i j}=\gamma_{00}+\gamma_{10} \text { trauma }_{i j}+\gamma_{20} \text { behavior }_{i j}+\gamma_{30} \text { family }_{i j}+\gamma_{40} \text { substance }_{i j} \\
+\gamma_{50} \text { age }_{i j}+\gamma_{60} \text { gender }_{i j}+u_{0 j}
\end{gathered}
$$

\section{Model interpretation.}

Multilevel models can answer a number of different research questions (Tabachnick \& Fidell, 2013). For the purposes of the present analysis, model interpretation first focused on the statistical significance and relative strength of each 
significant predictor. Each of the aforementioned research hypotheses could be rejected if the focus-of-treatment outcome variable was not significantly predicted by the associated CANS-derived scalar measure. Likewise, the relative strength of each predictor was compared to determine if trauma-related CANS items were more closely associated with the prescription of a trauma-focused treatment than other CANS items (and so forth for the other three outcome variables).

Next, the relationships between predictors and the outcome variables were interpreted in terms of the log odds. Given a one-unit increase on each of the predictor scales, how do the odds of being prescribed an associated treatment modality change? Odds ratios can also serve as an indication of effect size for the individual predictors (Tabachnick \& Fidell, 2013). Odds ratios were thus compared against the research hypotheses to determine if each CANS-derived predictor had the greatest effect in the prescription of an associated treatment outcome. 


\section{Conceptual Model}

Figure 3.1. Conceptual Model of the Study

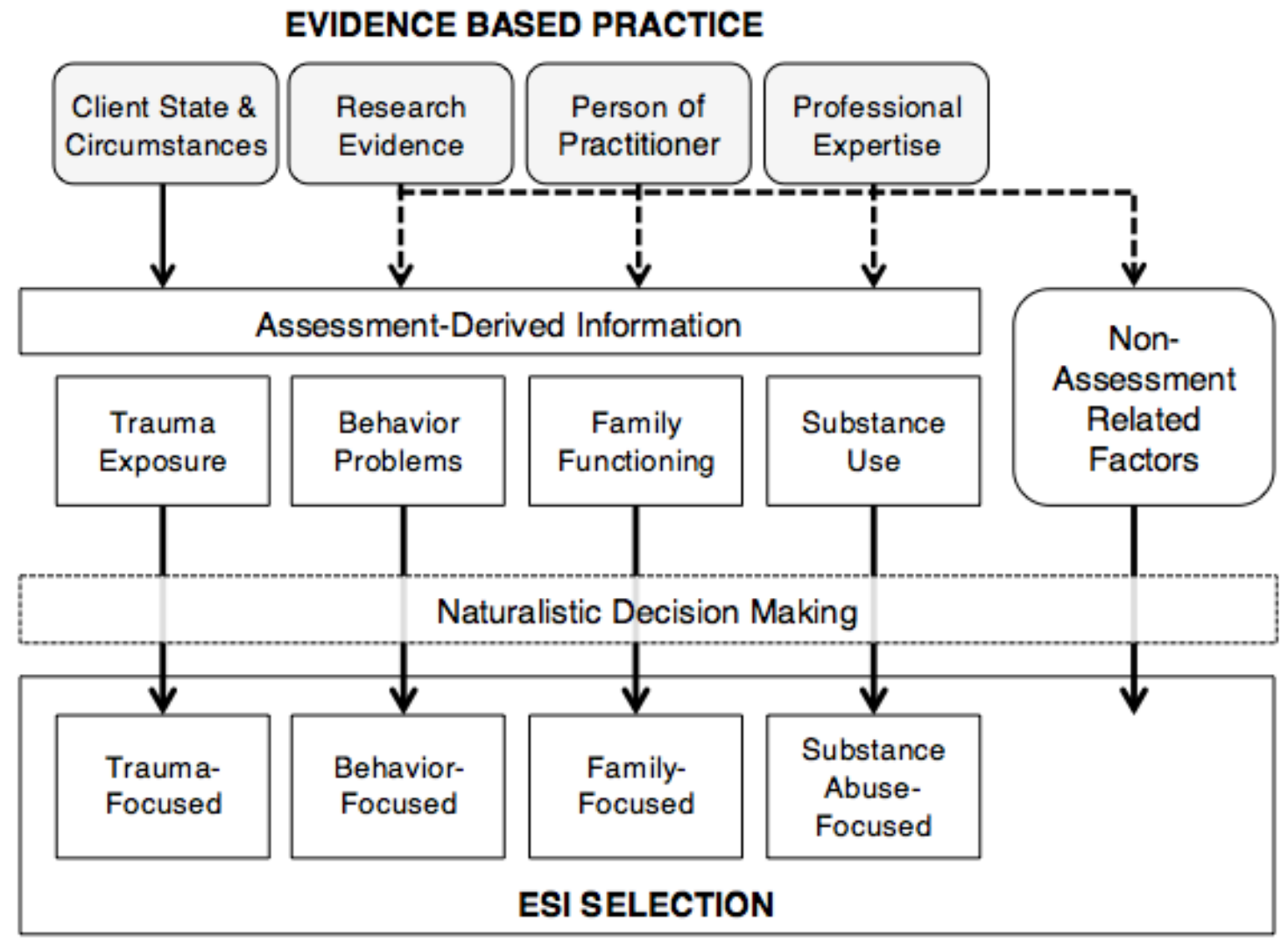

The visual conceptual model presented in Figure 3.1 is intended to provide a concise overview of the present study's purpose and framework. Project SAFESPACE sought to achieve the goal of assessment-driven, evidence-based treatment for children in out of home care. This study adopts an EBP perspective to evaluate the degree to which this goal is being achieved.

As detailed in the introductory chapter, assessment is an integral part of the EBP process. The four primary elements of the EBP model—client state and circumstances, research evidence, person of the practitioner, and professional expertise (see Gilgun, 
2005; Regehr et al., 2007) — are all shaped by the assessment process. The relationship between assessment and the client's state and circumstances is direct (denoted in the conceptual model by a solid line) as structured assessment is intended to provide a standardized approach for collecting and organizing information about the client and his or her environmental context. The relationship between assessment and the other elements is less direct but still interactive (denoted in the conceptual model by a dashed lined). For instance, assessment-derived information may frame the search for relevant research evidence, highlight potential areas of practitioner bias or values conflict (e.g., the client is assessed to have a history of sexual aggression), or provide context for drawing upon prior professional experiences and domains of expertise.

Responses on the CANS functional assessment were used to calculate four predictor variables: (1) trauma-exposure, (2) behavior problems, (3) family functioning, and (4) substance use. It is hypothesized that each of these dimensions will significantly predict a related ESI selection (e.g., trauma exposure will be a significant predictor of trauma-focused treatment).

The EBP model, NDM theory, and previous treatment decision-making research all suggest that client characteristics (as identified by the functional assessment) cannot account for all variability in treatment selection. Compelling research evidence suggests that an underlying schema may mediate the relationship between case conceptualization and subsequent treatment decisions. Similarly, the NDM framework's concept of situation-action pattern matching rules posits that treatment decisions rarely entail an exhaustive consideration of all possible alternatives. As such, it is entirely expected that non-assessment related factors will influence treatment recommendations and the 
association between assessment responses and ESI prescriptions may be weaker than anticipated. Keeping this in mind, multilevel modeling will be employed to examine the relative strength of each predictor - predicted relationship. To the extent that predictable relationships are observed, one may appraise the degree to which treatment prescriptions can be accurately described as assessment-driven and evidence-based. 


\section{CHAPTER 4:}

\section{RESULTS}

\section{Sample Description}

A total of 10,679 children have been screened as part of Project SAFESPACE as

of November $30^{\text {th }}, 2018$. Of these, $6,232(58.4 \%)$ positively screened-in and thus required a CANS assessment. However, SACWIS records only reflect 2,841 cases with one or more completed CANS assessments, suggesting a compliance rate of roughly $45.6 \%$.

Of the 2,841 cases with an initial CANS functional assessment, $436(15.3 \%)$ were children under the age of five; these cases were excluded from the analysis as they were assessed using a different version of the assessment instrument. An additional 14 cases were excluded because the incorrect CANS version had been administered (i.e., a child older than four years old was administered the KY CANS 0-4). An examination of missing data indicated that 49 CANS records were missing all item-level data and were thus deleted leaving a final sample size of 2342 cases with valid initial KY CANS 5+ assessments. Sample demographics are presented in Table 4.1. 
Table 4.1. Sample Demographics

\begin{tabular}{ll}
\hline \multicolumn{1}{c}{ Demographic characteristic } & $\mathrm{M}(\mathrm{SD})$ or $\%$ \\
\hline$(n=2342)$ & \\
Age & $12.4(3.7)$ \\
Gender & \\
Male & $50.3 \%$ \\
Female & $49.7 \%$ \\
Race/Ethnicity & \\
White & $74.9 \%$ \\
Biracial/Multiracial & $9.3 \%$ \\
Black/African American & $8.0 \%$ \\
Hispanic & $5.6 \%$ \\
Other/Unknown & $2.2 \%$ \\
\hline
\end{tabular}

Geographically, the sample spans the state of Kentucky with all nine DCBS

Protection and Permanency service regions being represented. The service region with the greatest representation is Salt River Trail $(n=483,20.6 \%)$. Eastern Mountain region has the fewest number of cases $(n=43,1.8 \%)$ included in the sample. Note that due to Project SAFESPACE's rolling implementation plan, not all service regions have been conducting assessments for an equivalent length of time.

About two-thirds of the children in the sample $(n=1538,65.7 \%)$ were experiencing their first OOHC episode at the time of data extraction. Slightly more than one-fifth $(n=497,21.2 \%)$ were in their second OOHC episode while less than one-tenth ( $n=197,8.3 \%)$ had already experienced two or more OOHC episodes prior to their current one. The maximum number of recorded OOHC episodes was eight $(n=2)$. These data were not available for $4.7 \%(n=110)$ of the sample. 


\section{Describing the Service Landscape}

A total of 381 unique clinicians completed the 2342 initial KY CANS 5+ assessments in the sample. The mean number of CANS completed per clinician is 6.15 $(S D=8.24)$. More than one-quarter $(n=103,26.5 \%)$ of clinicians completed only one initial CANS assessments among those cases included in the sample. Four clinicians completed 50 or more initial CANS assessments; the maximum number of assessments completed by a single clinician was 64 .

A total of 51 unique child-serving agencies are represented in the sample. Of these one is a child advocacy center (CAC); 14 are community mental health centers (CMHC); and 36 are private child caring agencies (PCC), private child placing (PCP) agencies, or agencies consisting of both PCC and PCP programs. Of all initial CANS assessments included in the sample, $0.1 \%(n=3)$ were completed at a CAC, $15.2 \%(n=$ 356) were completed at a CMHC, and $84.7 \%(n=1983)$ were completed at a PCC/PCP agency. The mean number of CANS completed per agency is $45.9(S D=90.6)$. Roughly $30 \%(n=15)$ of the agencies represented in the sample completed fewer than 10 initial CANS assessments. The five most heavily represented agencies account for more than $50 \%(n=1272)$ of the total initial CANS included in the sample. Agency five completed the greatest number of initial assessments $(n=517)$.

\section{Prescribed Treatment Modalities}

Of the 2342 cases for which an initial CANS assessment was completed, 2160 $(92.2 \%)$ resulted in a treatment recommendation. The majority of cases were assigned one treatment modality ( $n=1496,63.9 \%)$ while about one quarter of the sample was 
assigned two or more treatment modalities $(n=664,28.4 \%)$. The maximum number of treatment modalities assigned to a single case was $8(n=1)$.

The most frequently prescribed treatment modalities were cognitive-behavioral therapy $(n=1642,70.1 \%)$, trauma-focused cognitive-behavioral therapy $(n=542$, $23.1 \%)$, and cognitive therapy $(n=281,12.0 \%)$. For $125(5.3 \%)$ cases, the clinician selected the "other" option and identified a treatment modality not included in the dropdown list. The most frequent "other" treatment recommendations were play therapy ( $n=33,1.4 \%)$, solution-focused brief therapy $(n=29,1.2 \%)$, and person- (or client-) centered therapy $(n=22,0.9 \%)$.

Of the 381 clinicians who completed one or more initial CANS assessments, 82.9\% $(n=316)$ prescribed CBT at least one time, $52.2 \%(n=199)$ prescribed TF-CBT at least one time, and 36.2\% $(n=138)$ prescribed family therapy at least one time. About one-eighth $(n=47,12.3 \%)$ of all clinicians prescribed some form of "other" treatment modality. Only 9 (2.4\%) clinicians did not make any treatment recommendations; most of these $(n=7)$ had only completed one or two initial CANS assessments.

To examine the frequency of treatment prescriptions at an agency level, an agency was included in each modality's frequency count if one or more cases associated with that agency had been prescribed the respective modality. There were four modalities that had been prescribed at more than half of all included child-serving agencies: CBT $(n=$ 47, 92.2\%), TF-CBT ( $n=45,88.2 \%)$, cognitive therapy, $(n=34,66.7 \%)$ and family therapy $(n=33,64.7 \%)$. More than one-third $(n=20,39.2 \%)$ of all agencies were associated with one or more cases that had been prescribed an "other" modality. Table 
4.2 presents the complete list of all prescribed treatment modalities by cases, clinicians, and agencies.

The number of unique treatment modalities prescribed across all cases associated with a single child-serving agency ranged from 16 to 0 . As expected, there was a strong, positive correlation between the number of CANS completed by an agency and the total number of unique treatment modalities prescribed $(r=.589, p<0.001)$. However, there was an even more pronounced positive correlation between the total number of clinicians who completed the assessments associated with a given agency and the total number of unique treatment modalities prescribed $(r=.790, p<0.001)$. It is important to note that the total number of clinicians associated with an agency is not necessarily reflective of the size of the agency's clinical department. Table 4.3 presents the number of completed assessments, total number of associated clinicians, number of unique prescribed treatment modalities, and a list of prescribed modalities for each of the 51 agencies represented in the sample. 
Table 4.2. Frequency of Prescribed Treatment Modalities by Cases, Clinicians, and Agencies

\begin{tabular}{|c|c|c|c|c|c|c|}
\hline \multirow[b]{2}{*}{ Modality } & \multicolumn{2}{|c|}{$\begin{array}{c}\text { Cases } \\
(n=2342)\end{array}$} & \multicolumn{2}{|c|}{$\begin{array}{c}\text { Clinicians } \\
(n=381)\end{array}$} & \multicolumn{2}{|c|}{$\begin{array}{c}\text { Agencies } \\
(n=51)\end{array}$} \\
\hline & $\mathrm{n}$ & $\%$ & $\mathrm{n}$ & $\%$ & $\mathrm{n}$ & $\%$ \\
\hline Cognitive Behavioral Therapy & 1642 & $70.1 \%$ & 316 & $82.9 \%$ & 47 & $92.2 \%$ \\
\hline Trauma-Focused CBT & 542 & $23.1 \%$ & 199 & $52.2 \%$ & 45 & $88.2 \%$ \\
\hline Cognitive Therapy & 281 & $12.0 \%$ & 97 & $25.5 \%$ & 34 & $66.7 \%$ \\
\hline Family Therapy & 260 & $11.1 \%$ & 138 & $36.2 \%$ & 33 & $64.7 \%$ \\
\hline None & 182 & $7.8 \%$ & 9 & $2.4 \%$ & 2 & $3.9 \%$ \\
\hline Other (see below) & 125 & $5.3 \%$ & 47 & $12.3 \%$ & 20 & $39.2 \%$ \\
\hline Dialectical Behavior Therapy & 124 & $5.3 \%$ & 50 & $13.1 \%$ & 22 & $43.1 \%$ \\
\hline Seven Challenges & 71 & $3.0 \%$ & 23 & $6.0 \%$ & 13 & $25.5 \%$ \\
\hline Trauma Recovery \& Empowerment & 44 & $1.9 \%$ & 30 & $7.9 \%$ & 16 & $31.4 \%$ \\
\hline Parent-Child Interaction Therapy & 31 & $1.3 \%$ & 20 & $5.2 \%$ & 16 & $31.4 \%$ \\
\hline Structural Family Therapy & 23 & $1.0 \%$ & 15 & $3.9 \%$ & 10 & $19.6 \%$ \\
\hline Child-Parent Psychotherapy & 21 & $0.9 \%$ & 20 & $5.2 \%$ & 14 & $27.4 \%$ \\
\hline Restorative Approach & 17 & $0.7 \%$ & 12 & $3.1 \%$ & 7 & $13.7 \%$ \\
\hline Brief Strategic Family Therapy & 13 & $0.6 \%$ & 9 & $2.4 \%$ & 5 & $9.8 \%$ \\
\hline EMDR & 8 & $0.3 \%$ & 4 & $1.0 \%$ & 3 & $5.9 \%$ \\
\hline Wraparound & 6 & $0.3 \%$ & 4 & $1.0 \%$ & 4 & $7.8 \%$ \\
\hline Adolescent Community Reinforcement & 4 & $0.2 \%$ & 4 & $1.0 \%$ & 3 & $5.9 \%$ \\
\hline Prolonged Exposure Therapy & 1 & $<0.1 \%$ & 1 & $0.3 \%$ & 1 & $2.0 \%$ \\
\hline Sanctuary Model & 0 & $0 \%$ & 0 & $0 \%$ & 0 & $0 \%$ \\
\hline \multicolumn{7}{|l|}{ “Other" Modalities } \\
\hline Play Therapy & 33 & $1.4 \%$ & 20 & $5.2 \%$ & 10 & $19.6 \%$ \\
\hline Solution Focused (Brief) Therapy & 29 & $1.2 \%$ & 6 & $1.6 \%$ & 7 & $13.7 \%$ \\
\hline Person (Client) Centered Therapy & 22 & $0.9 \%$ & 8 & $2.1 \%$ & 3 & $5.9 \%$ \\
\hline Motivational Interviewing & 21 & $0.9 \%$ & 11 & $2.9 \%$ & 6 & $11.8 \%$ \\
\hline Reality Therapy & 9 & $0.4 \%$ & 7 & $1.8 \%$ & 6 & $11.8 \%$ \\
\hline Expressive Therapies & 7 & $0.3 \%$ & 5 & $1.3 \%$ & 4 & $7.8 \%$ \\
\hline Interactive Psychotherapy & 4 & $0.2 \%$ & 1 & $0.3 \%$ & 1 & $2.0 \%$ \\
\hline Seeking Safety & 4 & $0.2 \%$ & 4 & $1.0 \%$ & 2 & $3.9 \%$ \\
\hline Narrative Therapy & 3 & $0.1 \%$ & 3 & $0.8 \%$ & 2 & $3.9 \%$ \\
\hline Applied Behavior Analysis & 3 & $0.1 \%$ & 2 & $0.5 \%$ & 2 & $3.9 \%$ \\
\hline Operant Conditioning & 2 & $0.1 \%$ & 1 & $0.3 \%$ & 1 & $2.0 \%$ \\
\hline Behavior Modification & 1 & $<0.1 \%$ & 1 & $0.3 \%$ & 1 & $2.0 \%$ \\
\hline
\end{tabular}


Table 4.3. Completed CANS, Assessing Clinicians, and Prescribed Modalities by Agency

\begin{tabular}{|c|c|c|c|c|c|}
\hline Agency & Type & $\begin{array}{l}\text { CANS } \\
\text { (n) }\end{array}$ & $\begin{array}{l}\text { Clinicians } \\
(\mathrm{n})\end{array}$ & $\begin{array}{l}\text { Prescribed } \\
\text { modalities (n) }\end{array}$ & Prescribed modalities \\
\hline Agency \#18 & $\begin{array}{l}\mathrm{PCC} / \\
\mathrm{PCP}\end{array}$ & 70 & 15 & 16 & $\begin{array}{l}\text { BSFT, CPP, CT, CBT, DBT, FT, PCIT, RA, } \\
\text { SFT, TREM, TFCBT, PLAY, SFBT, } M I, E T, N T\end{array}$ \\
\hline Agency \#41 & $\begin{array}{l}\mathrm{PCC} / \\
\mathrm{PCP}\end{array}$ & 197 & 30 & 15 & $\begin{array}{l}\text { ACR, CT, CBT, DBT, EMDR, FT, PET, RA, } \\
\text { SFT, TREM, TFCBT, } P L A Y, M I, R T, S S\end{array}$ \\
\hline Agency \#26 & $\begin{array}{l}\mathrm{PCC} / \\
\mathrm{PCP}\end{array}$ & 65 & 18 & 14 & $\begin{array}{l}\text { BSFT, CPP, CT, CBT, DBT, EMDR, FT, } \\
\text { PCIT, RA, SC, SFT, TREM, TFCBT, PLAY }\end{array}$ \\
\hline Agency \#27 & PCP & 390 & 37 & 14 & $\begin{array}{l}\text { CPP, CT, CBT, DBT, FT, PCIT, SFT, TREM, } \\
\text { TFCBT, } P L A Y, P C T, M I, R T, N T\end{array}$ \\
\hline Agency \#5 & PCP & 517 & 36 & 13 & $\begin{array}{l}\text { CT, CBT, DBT, FT, PCIT, TREM, TFCBT, } \\
P L A Y, P C T, R T, I P, O C, B M O D\end{array}$ \\
\hline Agency \#25 & $\begin{array}{l}\text { CMHC/ } \\
\text { PCP }\end{array}$ & 75 & 29 & 11 & $\begin{array}{l}\text { CPP, CT, CBT, DBT, FT, PCIT, SFT, TREM, } \\
\text { TFCBT, SFBT, } M I\end{array}$ \\
\hline Agency \#10 & CMHC & 24 & 9 & 10 & $\begin{array}{l}\text { CT, CBT, FT, SC, SFT, TREM, TFCBT, } \\
P L A Y, M I, E T\end{array}$ \\
\hline Agency \#12 & $\mathrm{CMHC}$ & 18 & 6 & 9 & $\begin{array}{l}\text { BSFT, CPP, CT, CBT, DBT, FT, PCIT, } \\
\text { TFCBT, } E T\end{array}$ \\
\hline Agency \#15 & PCC & 89 & 10 & 9 & $\begin{array}{l}\text { CT, CBT, DBT, FT, SC, TREM, TFCBT, } \\
S F B T, R T\end{array}$ \\
\hline Agency \#23 & PCP & 11 & 6 & 9 & $\begin{array}{l}\text { CPP, CT, CBT, DBT, FT, PCIT, TREM, } \\
\text { TFCBT, WRAP }\end{array}$ \\
\hline Agency \#28 & PCP & 37 & 11 & 9 & $\begin{array}{l}\text { CPP, CT, CBT, FT, SC, TREM, TFCBT, } \\
S F B T, E T\end{array}$ \\
\hline Agency \#36 & $\begin{array}{l}\mathrm{PCC} / \\
\mathrm{PCP}\end{array}$ & 67 & 12 & 9 & $\begin{array}{l}\mathrm{CPP}, \mathrm{CT}, \mathrm{CBT}, \mathrm{FT}, \mathrm{PCIT}, \mathrm{SC}, \mathrm{TFCBT}, P L A Y, \\
P C T\end{array}$ \\
\hline Agency \#40 & $\begin{array}{l}\mathrm{PCC} / \\
\mathrm{PCP}\end{array}$ & 32 & 11 & 9 & $\begin{array}{l}\text { CT, CBT, DBT, FT, RA, SFT, TREM, TFCBT, } \\
P L A Y\end{array}$ \\
\hline Agency \#44 & $\begin{array}{l}\mathrm{PCC} / \\
\mathrm{PCP}\end{array}$ & 11 & 6 & 9 & $\begin{array}{l}\mathrm{ACR}, \mathrm{CPP}, \mathrm{CT}, \mathrm{CBT}, \mathrm{DBT}, \mathrm{FT}, \mathrm{SC}, \mathrm{TFCBT}, \\
R T\end{array}$ \\
\hline Agency \#3 & $\begin{array}{l}\mathrm{PCC} / \\
\mathrm{PCP}\end{array}$ & 42 & 5 & 8 & $\begin{array}{l}\text { BSFT, CT, CBT, DBT, FT, SFT, TREM, } \\
\text { TFCBT }\end{array}$ \\
\hline Agency \#7 & $\begin{array}{l}\mathrm{PCC} / \\
\mathrm{PCP}\end{array}$ & 16 & 5 & 8 & CT, CBT, DBT, FT, SC, SFT, TREM, TFCBT \\
\hline Agency \#14 & CMHC & 79 & 17 & 8 & $\begin{array}{l}\text { ACR, CT, CBT, FT, PCIT, SC, TFCBT, } \\
\text { WRAP }\end{array}$ \\
\hline Agency \#37 & $\begin{array}{l}\mathrm{PCC} / \\
\mathrm{PCP}\end{array}$ & 35 & 5 & 8 & CT, CBT, FT, PCIT, SC, SFT, TFCBT, PLAY \\
\hline Agency \#39 & PCP & 60 & 19 & 8 & BSFT, CBT, FT, SC, TREM, TFCBT, $M I, S S$ \\
\hline Agency \#6 & $\mathrm{CMHC}$ & 36 & 7 & 7 & CPP, CT, CBT, FT, PCIT, TREM, TFCBT \\
\hline Agency \#34 & CMHC & 35 & 11 & 7 & CT, CBT, FT, PCIT, SC, TFCBT, WRAP \\
\hline Agency \#43 & PCC & 14 & 4 & 7 & CT, CBT, DBT, FT, RA, TFCBT, WRAP \\
\hline Agency \#46 & $\begin{array}{l}\mathrm{PCC} / \\
\mathrm{PCP}\end{array}$ & 16 & 5 & 7 & CPP, CT, CBT, DBT, FT, PCIT, TFCBT \\
\hline Agency \#19 & $\begin{array}{l}\mathrm{PCC} / \\
\mathrm{PCP}\end{array}$ & 40 & 8 & 6 & CT, CBT, DBT, FT, RA, TFCBT \\
\hline Agency \#29 & PCP & 52 & 2 & 6 & CT, CBT, DBT, TFCBT, $S F B T, A B A$ \\
\hline Agency \#33 & PCC & 56 & 6 & 6 & CT, CBT, DBT, FT, TFCBT, $R T$ \\
\hline
\end{tabular}




\begin{tabular}{|c|c|c|c|c|c|}
\hline Agency \#45 & PCC & 20 & 2 & 6 & CT, CBT, DBT, FT, RA, TFCBT \\
\hline Agency \#1 & CMHC & 40 & 10 & 5 & CT, CBT, PCIT, SC, TFCBT \\
\hline Agency \#22 & PCC & 15 & 2 & 5 & CT, CBT, DBT, FT, TFCBT \\
\hline Agency \#30 & $\mathrm{CMHC}$ & 24 & 5 & 5 & CPP, CT, CBT, DBT, TFCBT \\
\hline Agency \#38 & CMHC & 2 & 2 & 5 & CPP, CBT, FT, PCIT, TFCBT \\
\hline Agency \#2 & $\begin{array}{l}\mathrm{PCC} / \\
\mathrm{PCP}\end{array}$ & 11 & 3 & 4 & CBT, TFCBT, PLAY, SFBT \\
\hline Agency \#11 & PCC & 4 & 2 & 4 & CBT, DBT, TREM, TFCBT \\
\hline Agency \#13 & $\mathrm{CMHC}$ & 7 & 6 & 4 & CBT, FT, PCIT, TFCBT \\
\hline Agency \#24 & PCC & 12 & 2 & 4 & CT, CBT, FT, SFBT \\
\hline Agency \#31 & $\mathrm{PCP}$ & 30 & 3 & 4 & CPP, CBT, FT, TFCBT \\
\hline Agency \#32 & PCC & 1 & 1 & 4 & CT, CBT, FT, TFCBT \\
\hline Agency \#42 & PCP & 13 & 1 & 4 & CT, CBT, EMDR, TFCBT \\
\hline Agency \#4 & PCC & 5 & 1 & 3 & CBT, FT, TFCBT \\
\hline Agency \#16 & $\begin{array}{l}\text { PCC/ } \\
\text { PCP }\end{array}$ & 16 & 2 & 3 & CT, CBT, TFCBT \\
\hline Agency \#51 & PCC & 4 & 1 & 3 & CT, CBT, TFCBT \\
\hline Agency \#9 & $\begin{array}{l}\mathrm{PCC} / \\
\mathrm{PCP}\end{array}$ & 2 & 2 & 2 & $\mathrm{CBT}, \mathrm{SC}$ \\
\hline Agency \#17 & PCP & 27 & 3 & 2 & CBT, TFCBT \\
\hline Agency \#35 & CMHC & 2 & 2 & 2 & CBT, TFCBT \\
\hline Agency \#47 & $\mathrm{CMHC}$ & 8 & 3 & 2 & CBT, TFCBT \\
\hline Agency \#48 & CAC & 3 & 2 & 2 & CBT, TFCBT \\
\hline Agency \#8 & PCP & 1 & 1 & 1 & TFCBT \\
\hline Agency \#20 & $\mathrm{PCP}$ & 3 & 1 & 1 & CBT \\
\hline Agency \#21 & PCC & 2 & 1 & 1 & $A B A$ \\
\hline Agency \#49 & $\mathrm{CMHC}$ & 5 & 1 & 0 & -- \\
\hline Agency \#50 & CMHC & 1 & 1 & 0 & -- \\
\hline \multicolumn{6}{|c|}{ 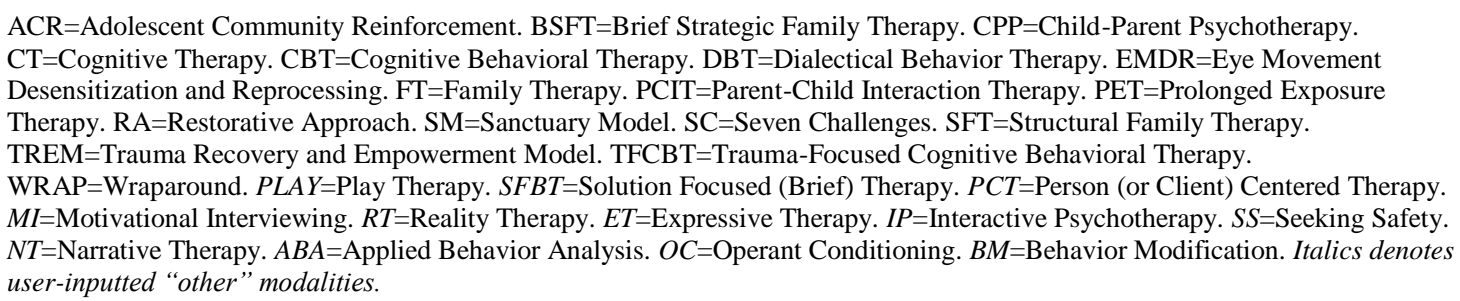 } \\
\hline
\end{tabular}


At the clinician-level, there is tremendous variability in the degree to which multiple types of treatment modalities are prescribed. A plurality of clinicians $(n=112$, 29.4\%) had prescribed two unique treatment modalities across all the initial CANS he or she completed; slightly fewer had prescribed only one treatment modality $(n=107$, $28.1 \%)$. Two clinicians $(0.5 \%)$ had prescribed 10 different treatment modalities - the maximum number in the present sample.

Raw frequencies of unique treatment modalities prescriptions are difficult to interpret given the differing number of initial CANS assessments completed among the clinicians associated with the sample. Clinicians who have completed a greater number of assessments will have had greater opportunity to assign different treatment recommendations than those who have completed fewer, irrespective of personal treatment decision making philosophies or proclivities. As such, examining the ratio of completed CANS to modalities assigned may provide more accurate insight. As illustrated in Table 4.4, the most homogenous treatment modality prescription pattern is that of clinician \#253 who has completed 55 initial CANS assessments and prescribed CBT for every case. At the other extreme, the most eclectic treatment modality prescription patterns are seen among those clinicians who have completed only one initial CANS assessment but have prescribed five or more unique modalities to that single case. 
Table 4.4. Most Homogenous and Eclectic Modality Prescriptions by Clinician

\begin{tabular}{|c|c|c|c|}
\hline Clinician & Agency & CANS : Modalities & Prescribed Modalities \\
\hline \multicolumn{4}{|c|}{$\underline{\text { Most Homogenous }}$} \\
\hline 253 & Agency \#5 & $55: 1$ & CBT \\
\hline 149 & Agency \#5 & $28: 1$ & CBT \\
\hline 131 & Agency \#41 & $22: 1$ & TFCBT \\
\hline 142 & Agency \#5 & $64: 3$ & CT, CBT, TFCBT \\
\hline 61 & Agency \#5 & $19: 1$ & CBT \\
\hline \multicolumn{4}{|c|}{$\underline{\text { Most Eclectic }}$} \\
\hline 320 & Agency \#36 & $1: 5$ & CT, CBT, FT, SC, TFCBT \\
\hline 236 & Agency \#41 & $1: 6$ & ACR, CBT, FT, RA, SFT, TREM \\
\hline 363 & Agency \#26 & $1: 6$ & CPP, CT, CBT, FT, RA, SC \\
\hline 20 & Agency \#10 & $1: 6$ & CT, CBT, FT, SFT, TREM, TFCBT \\
\hline 382 & Agency \#44 & $1: 7$ & ACR, BSFT, CT, CBT, DBT, FT, TFCBT \\
\hline \multicolumn{4}{|c|}{$\begin{array}{l}\text { ACR=Adolescent Community Reinforcement. BSFT=Brief Strategic Family Therapy. CPP=Child-Parent } \\
\text { Psychotherapy. } C \mathrm{CT}=\text { Cognitive Therapy. } \mathrm{CBT}=\text { Cognitive Behavioral Therapy. DBT=Dialectical Behavior Therapy. } \\
\text { FT=Family Therapy. RA=Restorative Approach. SC=Seven Challenges. SFT=Structural Family Therapy. } \\
\text { TREM=Trauma Recovery and Empowerment Model. TFCBT=Trauma-Focused Cognitive Behavioral Therapy. }\end{array}$} \\
\hline
\end{tabular}

\section{Focus-of-Treatment Composite Variables}

Based on the user-inputted responses when an "other" treatment modality was prescribed, three additional ESIs were identified as appropriate for inclusion in the focusof-treatment composite variables: child-centered play therapy, motivational interviewing, and seeking safety. The CEBC target population, scientific rating, and CWS relevance ratings, along with the focus-of-treatment inclusion categories are presented in Table 4.5. These modalities are in addition to those listed in Table 3.1. 
Table 4.5. “Other” ESIs \& Focus-of-Treatment Composite Variable Inclusion

\begin{tabular}{|c|c|c|c|c|c|c|c|}
\hline \multirow{2}{*}{ ESI } & \multirow{2}{*}{$\begin{array}{c}\text { Target } \\
\text { Population }\end{array}$} & \multirow{2}{*}{$\begin{array}{c}\text { CEBC } \\
\text { Scientific } \\
\text { Rating }\end{array}$} & \multirow{2}{*}{$\begin{array}{l}\text { CEBC CWS } \\
\text { Relevance }\end{array}$} & \multicolumn{4}{|c|}{ Focus-Of-Treatment Variable Inclusion } \\
\hline & & & & Trauma & Behavior & Family & $\begin{array}{c}\text { Sub } \\
\text { Abuse }\end{array}$ \\
\hline $\begin{array}{l}\text { Child-Centered } \\
\text { Play Therapy } \\
\text { (CCPT) }\end{array}$ & $\begin{array}{l}\text { Children (3-10) } \\
\text { who are } \\
\text { experiencing } \\
\text { social, emotional, } \\
\text { behavioral, and } \\
\text { relational } \\
\text { problems }\end{array}$ & Promising & Medium & & Y & & \\
\hline $\begin{array}{l}\text { Motivational } \\
\text { Interviewing } \\
\text { (MI) }\end{array}$ & $\begin{array}{l}\text { Caregivers of } \\
\text { children referred } \\
\text { to the child } \\
\text { welfare system; } \\
\text { has been used } \\
\text { with adolescents }\end{array}$ & Well-Supported & Medium & & $\mathrm{Y}$ & & $\mathrm{Y}$ \\
\hline $\begin{array}{l}\text { Seeking Safety } \\
\text { (SS) }\end{array}$ & $\begin{array}{l}\text { Adolescents with } \\
\text { a history of } \\
\text { trauma and/or } \\
\text { substance abuse }\end{array}$ & Promising & Medium & Y & & & Y \\
\hline
\end{tabular}

Behavior-focused ESIs were by far the most common treatment

recommendations; almost $90 \%$ of the total sample was prescribed an ESI intended to address emotional or behavioral concerns. Approximately one fourth $(24.8 \%, n=581)$ of the total sample had been prescribed a trauma-focused ESI. About one eighth $(12.7 \%, n=$ 298) of the sample had been prescribed some form of family therapy although less than $3 \%(n=65)$ were specific ESIs as described in the CEBC database. Substance usefocused ESIs were the least frequently prescribed with only $6.4 \%(n=150)$ of the sample being recommended a modality aimed at addressing alcohol or drug use. 


\section{Findings from the Initial CANS Assessment}

Table 4.6 presents the mean raw score for each of the six primary CANS domains.

Because the domains are not comprised of an equal number of items, the raw scores are not well-suited for cross domain comparison. As such, a standardized score was also calculated using the following formula: [(raw score $* 10) /$ number of items]. For each domain, standardized scores can range from 0 to 30, with higher values indicating a greater degree of problems.

Table 4.6. Mean Initial CANS Assessments Scores by Domain

\begin{tabular}{lcccccc}
\hline CANS Domain & Items & Raw Score & \multicolumn{2}{c}{ Raw Score } & \multicolumn{2}{c}{ Standardized Score } \\
Min - Max & M & $(S D)$ & M & $(S D)$ \\
\hline Child Strengths & 12 & $0-35$ & 15.17 & $(7.14)$ & 12.64 & $(5.95)$ \\
Life Domain Functioning & 15 & $0-38$ & 9.13 & $(5.25)$ & 6.09 & $(3.50)$ \\
Emotional/Behavioral Needs & 13 & $0-27$ & 7.52 & $(5.04)$ & 5.79 & $(3.88)$ \\
Caregiver Needs/Strengths & 20 & $0-60$ & 8.54 & $(11.20)$ & 4.27 & $(5.60)$ \\
Child Risk Behaviors & 13 & $0-26$ & 2.47 & $(3.14)$ & 1.90 & $(2.43)$ \\
Acculturation & 4 & $0-11$ & 0.37 & $(1.09)$ & .93 & $(2.72)$ \\
Total Instrument & 77 & $0-148$ & 43.20 & $(22.87)$ & 31.62 & $(16.14)$ \\
\hline
\end{tabular}

Based on mean standardized scores, the child strengths domain was assessed to be the most problematic among the sample $(M=12.64, S D=5.95)$. The domain with the second greatest mean standardized score was life domain functioning $(M=6.09, S D=$ 3.50). The acculturation domain $(M=0.93, S D=2.71)$ consists of only four items, and relatively few children were assessed to have actionable problems in this area.

\section{CANS modules.}

The KY CANS 5+ includes eight modules that are triggered by any non-zero response to a related item within one of the six primary domains. By examining the 
proportion of the completed functional assessments that resulted in an activation of each module, one can ascertain the extent to which the respective problem areas associated with each module have been identified within the total sample. The most frequently triggered module was trauma experiences with more than half $(58.9 \%, n=1380)$ of the sample requiring additional assessment in this area. Slightly more than half $(50.9 \%, n=$ 1191) of the sample also triggered the school module, which relates to the child's achievement, attendance, behavior, and interactions with teachers within their present academic setting. The sexually aggressive module was the least frequently triggered with only about $3 \%(n=78)$ of the sample requiring additional assessment. Table 4.7 presents the frequencies with which each module was triggered.

Table 4.7. Frequency of Triggered Modules Among Initial CANS Assessments

\begin{tabular}{lcc}
\hline CANS Module & $\mathrm{n}$ & $\%$ \\
\hline Trauma Experiences Module & 1380 & $58.9 \%$ \\
School Module & 1191 & $50.9 \%$ \\
Violence Module & 701 & $29.9 \%$ \\
Substance Use Module & 615 & $26.3 \%$ \\
Juvenile Justice (Delinquency) Module & 490 & $20.1 \%$ \\
Runaway Module & 410 & $17.5 \%$ \\
Intellectual/Developmental Needs Module & 339 & $14.5 \%$ \\
Sexually Aggressive Module & 78 & $3.3 \%$ \\
\hline
\end{tabular}

\section{Trauma experiences.}

As noted, more than half of all assessed cases $(58.9 \%, n=1380)$ triggered the trauma experiences module. Most of the sample $(55.3 \%, n=1296)$ showed some evidence of problems (i.e., a non-zero item response) related to at least one type of trauma experience. Among those cases assessed to have any trauma experiences, a greater number endorsed two $(n=295)$ or three $(n=258)$ different types of experiences 
than those who endorsed only one type of trauma experience $(n=248)$. More than onetenth $(11.1 \%, n=261)$ of the sample was assessed to have problems related to five or more unique types of trauma experiences. The most frequently endorsed trauma experiences were neglect $(44.7 \%, n=1048)$, witness to family violence $(30.7 \%, n=719)$, and emotional/verbal abuse $(27.7 \%, n=648)$.

Just under half $(45.5 \%, n=1065)$ of the total sample — and $77.1 \%$ of those cases that triggered the trauma module - evidenced trauma-related symptoms. Affect regulation $(28.4 \%, n=664)$, traumatic grief/separation $(27.8 \%, n=652)$, and intrusions $(25.9 \%, n=$ 606) affected the largest proportions of the sample per assessment responses.

Dissociation was the least prevalent trauma-related symptom with less than one in ten assessments $(8.7 \%, \mathrm{n}=203)$ suggesting any degree of dissociative experiences. More than $5 \%(n=73)$ of the youth who triggered the trauma experiences module were assessed to have problems related to all six of the included trauma symptoms.

The time before treatment item in the trauma adjustment sub-module records the amount of elapsed time between the trauma experience and its subsequent recognition and treatment. Among those cases that triggered the trauma experiences module, almost half $(49.3 \%, n=680)$ were assessed as having had their trauma recognized and treatment started within one month of the initial experience. However, assessments responses suggest that one-fifth of the sample $(19.8 \%, n=273)$ waited more than a year after the traumatic experience before it was recognized and treatment was initiated.

A composite trauma exposure score was calculated for each case using the 17 trauma-related CANS items listed in Table 4.8. Scores for cases that did not trigger the trauma experiences module were set to zero by default. Calculated trauma exposure 
scores ranged from 0 to 35 with higher scores indicating a greater prevalence and/or severity of trauma experiences and trauma-related symptoms. The mean trauma exposure score for the sample was $4.82(S D=5.85)$.

Table 4.8. Trauma-Related CANS Items

\begin{tabular}{|c|c|c|c|c|}
\hline \multirow[b]{2}{*}{ CANS Item } & \multicolumn{2}{|c|}{ Mean Response } & \multicolumn{2}{|c|}{ Percent of Sample Affected ${ }^{a}$} \\
\hline & M & $(S D)$ & $\%$ & $n$ \\
\hline \multicolumn{5}{|l|}{ Trauma Experiences Module } \\
\hline Neglect & 0.84 & $(1.06)$ & $44.7 \%$ & $(n=1048)$ \\
\hline Witness to Family Violence & 0.57 & $(0.93)$ & $30.7 \%$ & $(n=719)$ \\
\hline Emotional/Verbal Abuse & 0.47 & $(0.85)$ & $27.7 \%$ & $(n=648)$ \\
\hline Physical Abuse & 0.40 & $(0.74)$ & $25.8 \%$ & $(n=604)$ \\
\hline Sexual Abuse & 0.29 & $(0.65)$ & $19.3 \%$ & $(n=452)$ \\
\hline Witness/Victim to Criminal Activity & 0.19 & $(0.55)$ & $12.4 \%$ & $(n=291)$ \\
\hline Witness to Community Violence & 0.10 & $(0.41)$ & $6.6 \%$ & $(n=154)$ \\
\hline Medical Trauma & 0.05 & $(0.29)$ & $3.2 \%$ & $(n=74)$ \\
\hline Natural Disaster & 0.02 & $(0.17)$ & $1.4 \%$ & $(n=33)$ \\
\hline War Affected & $<0.01$ & $(0.08)$ & $0.2 \%$ & $(n=5)$ \\
\hline Terrorism Affected & $<0.01$ & $(0.04)$ & $0.04 \%$ & $(n=1)$ \\
\hline \multicolumn{5}{|l|}{$\underline{\text { Trauma Adjustment Sub-Module }}$} \\
\hline Affect Regulation & 0.41 & $(0.71)$ & $28.4 \%$ & $(n=664)$ \\
\hline Traumatic Grief/Separation & 0.39 & $(0.70)$ & $27.8 \%$ & $(n=652)$ \\
\hline Intrusions & 0.38 & $(0.71)$ & $25.9 \%$ & $(n=606)$ \\
\hline Attachment & 0.29 & $(0.57)$ & $23.3 \%$ & $(n=545)$ \\
\hline Avoidance & 0.35 & $(0.70)$ & $23.2 \%$ & $(n=543)$ \\
\hline Dissociation & 0.10 & $(0.36)$ & $8.7 \%$ & $(n=203)$ \\
\hline
\end{tabular}

\section{Emotional/behavioral problems.}

The vast majority of the sample $(93.5 \%, n=2190)$ was assessed to have one or more emotional/behavioral needs; for well over half of the cases $(60.1 \%, n=1427)$ these needs were rated at an actionable level (i.e., an item score of 2 or 3). On average, more than four emotional/behavioral needs $(M=4.33, S D=2.42)$ were identified as posing some degree of problems for each assessed case. The most frequently assessed emotional/behavioral needs included anxiety/worry $(66.4 \%, n=1555)$, 
impulsivity/hyperactivity $(61.6 \%, n=1442)$, oppositional behavior $(60.8 \%, n=1423)$, anger control $(60.1 \%, n=1408)$, and depression $(50.2 \%, n=1176)$.

Most of the sample $(64.9 \%, n=1521)$ evidenced either a history of or current problems related to at least one risk behavior. In terms of specific risk behaviors, the behaviors most frequently assessed to be historically or currently problematic were violence or aggression directed at others $(29.9 \%, n=701)$, intentional misbehavior (26.4\%, $n=619)$, self-injurious behaviors (22.3\%, $n=523)$, and delinquent behaviors $(20.9 \%, n=490)$. A significant positive correlation was observed between the number of emotional/behavioral needs identified and the number of specific risk behaviors assessed to be problematic for each case $(r=.595, p<0.001)$.

A composite emotional/behavioral problems score was calculated for each case using the 24 applicable CANS items presented in Table 4.9. Calculated scores ranged from 0 to 48 with higher scores reflecting a greater prevalence and/or severity of assessed emotional/behavioral problems. The mean emotional/behavioral problems scale score for the sample was $8.68(S D=6.83)$. 
Table 4.9. Emotional/Behavioral Problems-Related CANS Items

\begin{tabular}{|c|c|c|c|c|}
\hline \multirow[b]{2}{*}{ CANS Item } & \multicolumn{2}{|c|}{ Mean Response } & \multicolumn{2}{|c|}{ Percent of Sample Affected a } \\
\hline & M & $(S D)$ & $\%$ & $n$ \\
\hline \multicolumn{5}{|c|}{$\underline{\text { Child Emotional/Behavioral Needs }}$} \\
\hline Impulsivity/Hyperactivity & 0.97 & $(0.92)$ & $61.6 \%$ & $(n=1442)$ \\
\hline Oppositional & 0.94 & $(0.92)$ & $60.8 \%$ & $(n=1423)$ \\
\hline Anger Control & 0.91 & $(0.88)$ & $60.1 \%$ & $(n=1408)$ \\
\hline Anxiety/Worry & 0.90 & $(0.77)$ & $66.4 \%$ & $(n=1555)$ \\
\hline Attention & 0.69 & $(0.82)$ & $48.0 \%$ & $(n=1123)$ \\
\hline Depression & 0.64 & $(0.73)$ & $50.2 \%$ & $(n=1176)$ \\
\hline Conduct & 0.53 & $(0.79)$ & $37.1 \%$ & $(n=869)$ \\
\hline Attachment & 0.40 & $(0.68)$ & $30.1 \%$ & $(n=704)$ \\
\hline Somatization & 0.10 & $(0.34)$ & $8.6 \%$ & $(n=202)$ \\
\hline Psychosis & 0.06 & $(0.31)$ & $4.9 \%$ & $(n=114)$ \\
\hline Eating Disturbances & 0.06 & $(0.27)$ & $5.0 \%$ & $(n=118)$ \\
\hline \multicolumn{5}{|l|}{$\underline{\text { Child Risk Behaviors }}$} \\
\hline Danger to Others & 0.41 & $(0.69)$ & $29.9 \%$ & $(n=701)$ \\
\hline Intentional Misbehavior & 0.37 & $(0.70)$ & $26.4 \%$ & $(n=619)$ \\
\hline Delinquent Behaviors & 0.28 & $(0.60)$ & $20.9 \%$ & $(n=490)$ \\
\hline Self-Injurious Behaviors & 0.27 & $(0.55)$ & $22.3 \%$ & $(n=523)$ \\
\hline Suicide & 0.24 & $(0.56)$ & $19.2 \%$ & $(n=449)$ \\
\hline Runaway & 0.24 & $(0.60)$ & $17.5 \%$ & $(n=410)$ \\
\hline Bullying & 0.20 & $(0.53)$ & $14.4 \%$ & $(n=337)$ \\
\hline Other Self-Harm & 0.14 & $(0.45)$ & $11.0 \%$ & $(n=257)$ \\
\hline Sexually Reactive Behavior & 0.13 & $(0.25)$ & $10.0 \%$ & $(n=235)$ \\
\hline Exploited & 0.09 & $(0.38)$ & $6.4 \%$ & $(n=149)$ \\
\hline Sexual Aggression & 0.05 & $(0.28)$ & $3.3 \%$ & $(n=78)$ \\
\hline Fire Setting & 0.03 & $(0.21)$ & $2.0 \%$ & $(n=49)$ \\
\hline Commercial Sexual Exploitation & 0.02 & $(0.19)$ & $1.2 \%$ & $(n=27)$ \\
\hline
\end{tabular}

\section{Family functioning.}

The CANS functional assessment provides an opportunity to assess family functioning in relation to both the child and the caregiver role. From the child-level assessment items, impaired family functioning appears almost ubiquitous with $84.8 \%$ ( $n$ $=1986$ ) of cases assessed to have moderate, significant, or profound levels of family problems. Similarly, more than half of all cases $(61.8 \%, n=1448)$ report some degree of problems with functioning within the current living situation (which may include but is not limited to the child's biological family). By contrast, assessments of child strengths 
suggests that only $12.7 \%(n=297)$ of the sample displays significant nuclear family strengths as indicated by mutual love, respect, and the child's involvement in family activities. Just slightly more children $(14.7 \%, n=344)$ were assessed to have wellestablished, involved, and significantly supportive extended family relationships.

Caregiver-focused CANS items indicate that more than three-fifths $(62.6 \%, n=$ 1465) of the sample's caregivers had at least one assessed need. An average of almost five $(M=4.99, S D=5.56)$ different needs were assessed with a nonzero rating among all caregivers. More than half of those caregivers with at least one assessed need (i.e., the total score for the caregiver needs/strengths domain was greater than zero) evidenced some degree of need in eight or more different areas. The most frequently occurring caregiver needs included parental knowledge $(47.6 \%, n=1114)$, providing appropriate supervision $(46.0 \%, n=1078)$, being involved in the child's care $(45.6 \%, n=1078)$, and accessing social resources (i.e., friends and family) to assist with child rearing $(37.7 \%, n$ $=882$ ).

A composite family functioning score was calculated for each case using the 24 child- and caregiver-focused CANS items presented in Table 4.10. Calculated scores ranged from 0 to 72 with higher scores reflecting a greater prevalence and/or severity of assessed problems related to family functioning. The mean family functioning scale score for the sample was $14.66(S D=12.64)$. 
Table 4.10. Family Functioning-Related CANS Items

\begin{tabular}{|c|c|c|c|c|}
\hline \multirow[b]{2}{*}{ CANS Item } & \multicolumn{2}{|c|}{ Mean Response } & \multicolumn{2}{|c|}{ Percent of Sample Affected ${ }^{a}$} \\
\hline & M & $(S D)$ & $\%$ & $n$ \\
\hline \multicolumn{5}{|l|}{$\underline{\text { Life Domain Functioning }}$} \\
\hline Family Functioning & 1.76 & $(1.03)$ & $84.8 \%$ & $(n=1986)$ \\
\hline Living Situation & 1.08 & $(1.05)$ & $61.8 \%$ & $(n=1448)$ \\
\hline \multicolumn{5}{|l|}{$\underline{\text { Child Strengths }}$} \\
\hline Nuclear Family Strengths & 1.66 & $(0.90)$ & $87.3 \%$ & $(n=2045)$ \\
\hline Extended Family Relationships & 1.61 & $(0.96)$ & $85.3 \%$ & $(n=1998)$ \\
\hline \multicolumn{5}{|l|}{$\underline{\text { Caregiver Needs/Strengths }}$} \\
\hline Supervision & 0.83 & $(1.03)$ & $46.0 \%$ & $(n=1078)$ \\
\hline Involvement With Care & 0.81 & $(1.01)$ & $45.6 \%$ & $(n=1068)$ \\
\hline Knowledge & 0.74 & $(0.91)$ & $47.6 \%$ & $(n=1114)$ \\
\hline Social Resources & 0.63 & $(0.92)$ & $37.7 \%$ & $(n=882)$ \\
\hline Resources & 0.57 & $(0.91)$ & $33.5 \%$ & $(n=785)$ \\
\hline Financial Resources & 0.57 & $(0.90)$ & $34.4 \%$ & $(n=806)$ \\
\hline Substance Use & 0.51 & $(0.94)$ & $26.4 \%$ & $(n=618)$ \\
\hline Residential Stability & 0.47 & $(0.88)$ & $26.1 \%$ & $(n=612)$ \\
\hline Organization & 0.44 & $(0.83)$ & $27.0 \%$ & $(n=633)$ \\
\hline Safety & 0.43 & $(0.86)$ & $24.0 \%$ & $(n=563)$ \\
\hline Marital/Partner Violence & 0.38 & $(0.82)$ & $21.2 \%$ & $(n=496)$ \\
\hline Legal & 0.35 & $(0.79)$ & $19.2 \%$ & $(n=450)$ \\
\hline Accessibility to Child Care Services & 0.32 & $(0.71)$ & $20.4 \%$ & $(n=478)$ \\
\hline Transportation & 0.32 & $(0.75)$ & $18.2 \%$ & $(n=427)$ \\
\hline Mental Health & 0.30 & $(0.68)$ & $18.7 \%$ & $(n=437)$ \\
\hline Self-Care/Daily Living & 0.23 & $(0.66)$ & $12.9 \%$ & $(n=302)$ \\
\hline Educational Attainment & 0.21 & $(0.67)$ & $10.7 \%$ & $(n=251)$ \\
\hline Physical Health & 0.20 & $(0.58)$ & $12.6 \%$ & $(n=295)$ \\
\hline Posttraumatic Reactions & 0.18 & $(0.52)$ & $13.1 \%$ & $(n=306)$ \\
\hline Developmental & 0.07 & $(0.07)$ & $4.1 \%$ & $(n=97)$ \\
\hline
\end{tabular}

\section{Substance use.}

More than a quarter of all assessed cases $(26.3 \%, n=615)$ triggered the substance use module indicating some degree of concern regarding the child's use of drugs and/or alcohol (the CANS assessment does not document the specific substance being used). Approximately one in every seven cases $(15.3 \%, n=464)$ had a history of substance use and had only been abstinent for 30 days or less; about $5 \%(n=106)$ of the total sample was still actively using substances at the time of assessment. Among those cases who 
were assessed to have substance-related problems, more than three quarters $(75.4 \%, n=$ 464) had been using substances for at least one year. Most of these cases reported being negatively influenced to some degree by their peers or social networks $(69.8 \%, n=429)$, their parents $(57.4 \%, n=353)$, and their environments $(52.2 \%, n=321)$. About one third $(34.3 \%, n=211)$ of youth assessed to have substance-related concerns were in the maintenance stage of recovery, roughly an equal proportion $(34.8 \%, n=214)$ were in the treatment stage, while the remaining third were either in the contemplation stage $(19.5 \%$, $n=120)$ or in a state of denial about the problem $(11.4 \%, n=70)$.

A composite substance use score was calculated for each case using the 7 substance use-related CANS items listed in Table 4.11. Scores for cases that did not trigger the substance use module were set to zero by default. Calculated substance use scores ranged from 0 to 21 with higher scores indicating a greater prevalence and/or severity of substance use-related problems. The mean substance use score for the sample was $2.03(S D=4.01)$.

Table 4.11. Substance Use-Related CANS Items

\begin{tabular}{|c|c|c|c|c|}
\hline \multirow[b]{2}{*}{ CANS Item } & \multicolumn{2}{|c|}{ Mean Response } & \multicolumn{2}{|c|}{ Percent of Sample Affected ${ }^{a}$} \\
\hline & $\mathrm{M}$ & $(S D)$ & $\%$ & $n$ \\
\hline \multicolumn{5}{|c|}{$\underline{\text { Child Emotional/Behavioral Needs }}$} \\
\hline Substance Use & 0.37 & $(0.69)$ & $26.3 \%$ & $(n=615)$ \\
\hline \multicolumn{5}{|c|}{ Substance Use Module } \\
\hline Duration of Use & 0.35 & $(0.76)$ & $19.9 \%$ & $(n=466)$ \\
\hline Stage of Recovery & 0.29 & $(0.70)$ & $17.3 \%$ & $(n=405)$ \\
\hline Peer Influences & 0.28 & $(0.65)$ & $18.3 \%$ & $(n=429)$ \\
\hline Parental Influences & 0.28 & $(0.71)$ & $15.1 \%$ & $(n=353)$ \\
\hline Severity of Use & 0.26 & $(0.58)$ & $19.8 \%$ & $(n=464)$ \\
\hline Environmental Influences & 0.21 & $(0.60)$ & $13.7 \%$ & $(n=321)$ \\
\hline
\end{tabular}




\section{Bivariate Correlation Among Composite Predictor Variables}

Table 4.12. Correlation Matrix of CANS-Derived Predictor Variables

\begin{tabular}{lllll}
\hline & Trauma Exposure & $\begin{array}{l}\text { Behavioral } \\
\text { Problems }\end{array}$ & $\begin{array}{l}\text { Family } \\
\text { Functioning }\end{array}$ & Substance Use \\
\hline $\begin{array}{l}\text { Trauma Exposure } \\
\text { Behavioral }\end{array}$ & 1.000 & $0.479^{*}$ & 1.000 & \\
$\begin{array}{l}\text { Problems } \\
\begin{array}{l}\text { Family } \\
\text { Functioning }\end{array}\end{array}$ & $0.316^{*}$ & $0.237^{*}$ & 1.000 & \\
$\begin{array}{l}\text { Substance Use } \\
* p<0.001\end{array}$ & $0.110^{*}$ & $0.341^{*}$ & $0.127^{*}$ & 1.000 \\
\hline
\end{tabular}

As expected, a small to moderate positive correlation was observed among all CANS-derived composite predictor variables; all bivariate correlations were statistically significant. The strongest relationship was observed between trauma exposure and emotional/behavioral problems $(r=0.479, p<0.001)$. By contrast, trauma exposure and substance use had the weakest correlation $(r=0.110, p<0.001)$. Though statistically significant correlations were observed across all bivariate relationships, all are well below cutoffs suggesting concerns about multicollinearity (Meyers et al., 2013; Tabachnick \& Fidell, 2013).

\section{Normality of Composite Predictor Variable Scores}

Graphical and statistical examination of the CANS-derived composite predictor variables suggested nonnormal distributions. Specifically, each distribution was positively skewed due to the frequency of zero values within each calculated score. Multilevel logistic regression is not predicated upon assumptions about the distribution of predictor variables (Tabachnick \& Fidell, 2013). While the $t$ test does assume a normal distribution of the dependent variable, it is robust to normality violations with large samples of approximately equal group sizes (Wright, 1997), and was thus used in the 
subsequent examination of group differences by gender. In cases where the group size was not approximately equal (i.e., race/ethnicity), a Mann-Whitney test was employed. Because an analysis of variance may not be as powerful as nonparametric tests when the dependent variable is highly skewed (Wright, 1997), a Kruskal-Wallis test was used to examine group differences in predictor variable scores among age quartiles.

\section{Child-Level Group Differences in Composite Predictor Variable Scores}

On average, female clients scored significantly higher on the trauma exposure composite variable $(M=5.36, S D=6.08)$ than did male clients $(M=4.29, S D=5.57)$. Conversely, males had significantly greater mean scores on the emotional/behavioral scale (9.05 vs. 8.30$)$ and substance use scale (3.70 vs. 2.19$)$. There was not a statistically significant difference between genders in terms of family functioning scores.

To examine differences based on the child's age, the sample was first grouped into quartiles based on the age (in whole years) at the time of the initial CANS assessment. Statistically significant differences were observed in the mean rank score on the trauma exposure, emotional/behavior problems, and substance use scales. For ease of interpretation mean scores, rather than mean ranks, are presented in Table 4.13. The highest mean trauma exposure score was observed in the 10 to 13 year old group $(M=$ $5.10, S D=5.95)$. On average, children in the 14 to 15 year old group scored the highest on the emotional/behavioral problems scale $(M=10.2, S D=7.61)$. The oldest childrenthose older than 16 - scored the highest on the substance use scale $(M=4.52, S D=5.05)$ while average scores for children 9 years old or younger were negligible $(M=0.05, S D=$ $0.66)$. 
Due to the demographic composition of the sample, group differences by race/ethnicity were examined dichotomously between those clients identified as white and those identified as a racial or ethnic minority. No statistically significant racial/ethnic group differences were observed in the mean rank score across any of the four composite predictor variables.

Table 4.13. Child-Level Group Differences in CANS-Derived Predictor Variable Scores

\begin{tabular}{|c|c|c|c|c|c|c|c|c|}
\hline & \multicolumn{2}{|c|}{ Trauma Exposure } & \multicolumn{2}{|c|}{ Behavior Problems } & \multicolumn{2}{|c|}{$\begin{array}{c}\text { Family } \\
\text { Functioning }\end{array}$} & \multicolumn{2}{|c|}{ Substance Use } \\
\hline & M & $(S D)$ & M & $(S D)$ & M & $(S D)$ & M & $(S D)$ \\
\hline \multicolumn{9}{|l|}{ Gender $(n)$} \\
\hline Female (1163) & 5.36 & $(6.08)$ & 8.30 & $(6.77)$ & 14.44 & $(12.49)$ & 1.86 & $(3.70)$ \\
\hline \multirow[t]{2}{*}{ Male (1179) } & 4.29 & (5.57) & 9.05 & $(6.86)$ & 14.87 & (12.79) & 2.19 & $(4.28)$ \\
\hline & \multicolumn{2}{|c|}{$(t=-4.43, p<0.001)^{*}$} & \multicolumn{2}{|c|}{$(t=2.66, p=0.008)$} & \multicolumn{2}{|c|}{ n.s. } & \multicolumn{2}{|c|}{$(t=2.03, p=0.043)^{*}$} \\
\hline \multicolumn{9}{|l|}{ Age at CANS $(n)$} \\
\hline $5-9(599)$ & 5.00 & $(5.75)$ & 7.28 & $(6.15)$ & 15.36 & $(13.30)$ & 0.05 & $(0.66)$ \\
\hline $10-13(622)$ & 5.10 & $(5.95)$ & 7.97 & $(6.57)$ & 15.01 & $(13.06)$ & 0.48 & $(1.96)$ \\
\hline $14-15$ (537) & 4.77 & $(6.03)$ & 10.20 & (7.61) & 14.30 & (11.93) & 3.31 & $(4.68)$ \\
\hline \multirow[t]{2}{*}{$16-19$ (584) } & 4.39 & $(5.67)$ & 9.48 & $(6.61)$ & 13.90 & $(12.11)$ & 4.52 & $(5.05)$ \\
\hline & \multicolumn{2}{|c|}{$(H=9.01, p=0.029)$} & \multicolumn{2}{|c|}{$(H=71.7, p<0.001)$} & \multicolumn{2}{|c|}{ n.s. } & \multicolumn{2}{|c|}{$(H=666.9, p<0.001$} \\
\hline \multicolumn{9}{|l|}{$\underline{\text { Race/Ethnicity }(n)}$} \\
\hline White (1755) & 4.85 & $(5.92)$ & 8.60 & $(6.78)$ & 14.95 & $(12.96)$ & 1.99 & $(4.03)$ \\
\hline \multirow{2}{*}{ Minority (587) } & 4.73 & $(5.65)$ & 8.93 & $(6.97)$ & 13.77 & (11.59) & 2.15 & $(3.94)$ \\
\hline & \multicolumn{2}{|c|}{ n.s. } & \multicolumn{2}{|c|}{ n.s. } & \multicolumn{2}{|c|}{ n.s. } & \multicolumn{2}{|c|}{ n.s. } \\
\hline * Equal variances $n$ & Imed & & & & & & & \\
\hline
\end{tabular}




\section{Differences in CANS Domain Scores by Prescribed ESI}

Before modeling the ability of selected CANS response patterns to predict different treatment recommendations, it is of interest to examine differences in domain scores among cases who have or have not been prescribed the most frequently prescribed ESIs. Differences were analyzed using independent samples $t$ tests, correcting for violations of equal variances where necessary. It is important to note that these findings are merely descriptive in nature and should not be interpreted as a causal explanation for the prescription of different treatment modalities.

Table 4.14. Differences in Standardized CANS Domain Scores By Cases Prescribed/Not Prescribed Selected Modalities

\begin{tabular}{|c|c|c|c|c|c|c|}
\hline Treatment & $\begin{array}{l}\text { Child } \\
\text { Strengths }\end{array}$ & $\begin{array}{l}\text { Life Domain } \\
\text { Functioning }\end{array}$ & $\begin{array}{c}\text { Emotional / } \\
\text { Behavioral } \\
\text { Needs }\end{array}$ & $\begin{array}{c}\text { Caregiver } \\
\text { Needs / } \\
\text { Strengths }\end{array}$ & $\begin{array}{c}\text { Risk } \\
\text { Behaviors }\end{array}$ & Acculturation \\
\hline Recommendation & $\mathrm{M} \quad(S D)$ & $\mathrm{M} \quad(S D)$ & $\mathrm{M} \quad(S D)$ & $\mathrm{M} \quad(S D)$ & $\mathrm{M} \quad(S D)$ & $\mathrm{M} \quad(S D)$ \\
\hline \multicolumn{7}{|l|}{ Any Treatment } \\
\hline Yes $(\mathrm{n}=2160)$ & $12.7(5.9)^{* *}$ & $6.2(3.4)^{*}$ & $6.0(3.9)^{*}$ & $4.3(5.6)$ & $2.0(2.5)^{*}$ & $0.8(2.3)$ \\
\hline No $(n=182)$ & $11.7(6.6)$ & $4.8(3.9)$ & $3.6(3.4)$ & $3.7(5.5)$ & $0.8(1.4)$ & $0.9(2.8)$ \\
\hline \multicolumn{7}{|l|}{ CBT } \\
\hline Yes $(n=1642)$ & $12.6(6.1)$ & $6.2(3.4)^{* *}$ & $5.9(3.8)$ & $4.0(5.3)^{*}$ & $2.0(2.4)^{*}$ & $1.0(2.8)$ \\
\hline No $(n=700)$ & $12.6(5.9)$ & $5.8(3.7)$ & $5.6(3.9)$ & $4.9(6.2)$ & $1.6(2.4)$ & $0.8(2.6)$ \\
\hline \multicolumn{7}{|l|}{$\underline{\text { TF-CBT }}$} \\
\hline Yes $(\mathrm{n}=542)$ & $13.5(5.6)^{*}$ & $6.8(3.6)^{*}$ & $7.6(3.9)^{*}$ & $5.6(6.4)^{*}$ & $2.6(2.9)^{*}$ & $1.1(3.0)$ \\
\hline No $(n=1800)$ & $12.4(6.0)$ & $5.9(3.4)$ & $5.2(3.7)$ & $3.9(5.3)$ & $1.7(2.2)$ & $0.9(2.6)$ \\
\hline \multicolumn{7}{|l|}{ Cognitive Therapy } \\
\hline Yes $(\mathrm{n}=281)$ & $12.7(6.2)$ & $6.4(3.8)$ & $6.3(4.2)^{* *}$ & $4.4(5.9)$ & $2.2(2.7)$ & $0.9(2.7)$ \\
\hline No $(n=2061)$ & $12.6(5.9)$ & $6.0(3.5)$ & $5.7(3.8)$ & $4.3(5.6)$ & $1.9(2.4)$ & $0.9(2.7)$ \\
\hline \multicolumn{7}{|l|}{ Family Therapy } \\
\hline Yes $(n=260)$ & $14.2(5.4)^{*}$ & $7.9(3.6)^{*}$ & $8.0(3.9)^{*}$ & $5.3(5.8)^{*}$ & $3.1(3.0)^{*}$ & $0.9(2.4)$ \\
\hline No $(n=2082)$ & $12.4(6.0)$ & $5.9(3.4)$ & $5.5(3.8)$ & $4.1(5.6)$ & $1.7(2.3)$ & $0.9(2.8)$ \\
\hline \multicolumn{7}{|l|}{$\underline{\mathrm{DBT}}$} \\
\hline Yes $(n=124)$ & $14.3(5.9)^{* *}$ & $7.7(3.9)^{*}$ & $8.2(4.1)^{*}$ & $5.0(5.5)$ & $4.1(3.4)^{*}$ & $1.1(2.6)$ \\
\hline No $(n=2218)$ & $12.6(5.9)$ & $6.0(3.5)$ & $5.6(3.8)$ & $4.2(5.6)$ & $1.8(2.3)$ & $0.9(2.7)$ \\
\hline \multicolumn{7}{|l|}{ Seven Challenges } \\
\hline Yes $(\mathrm{n}=71)$ & $14.8(4.8)^{*}$ & $9.5(3.2)^{*}$ & $9.2(3.8)^{*}$ & $5.8(5.4)^{* *}$ & $4.8(3.5)^{*}$ & $1.5(3.4)$ \\
\hline No $(\mathrm{n}=2271)$ & $12.6(6.0)$ & $6.0(3.5)$ & $5.7(3.8)$ & $4.2(5.6)$ & $1.8(2.3)$ & $0.9(2.7)$ \\
\hline
\end{tabular}


First, standardized mean CANS domain scores were compared between those cases who had been prescribed any modality and those not recommended for any form of treatment. Children who had been assigned any treatment modality evidenced significantly greater mean scores in child strengths (12.7 vs. 11.7), life domain functioning (6.2 vs. 4.8), emotional/behavioral needs ( 6.0 vs. 3.6), and risk behaviors (2.0 vs. 0.8) domains. Similar comparisons were made for the six most frequently assigned treatment modalities and are presented in Table 4.14.

\section{Multilevel Logistic Regression Modeling}

The unconditional model (Model 0) and the intraclass correlation.

Table 4.15. Unconditional Model (Model 0) Coefficients

\begin{tabular}{|c|c|c|c|c|}
\hline & \multicolumn{4}{|c|}{ Outcome Variable } \\
\hline & $\begin{array}{l}\text { Trauma- } \\
\text { Focused }\end{array}$ & $\begin{array}{l}\text { Behavior- } \\
\text { Focused }\end{array}$ & $\begin{array}{l}\text { Family- } \\
\text { Focused }\end{array}$ & $\begin{array}{l}\text { Substance Use- } \\
\text { Focused }\end{array}$ \\
\hline Intercept & $-1.022(0.106)^{*}$ & $2.557(0.124)^{*}$ & $-1.876(0.116)^{*}$ & $-3.116(0.166)^{*}$ \\
\hline Variance & $2.305(0.295)^{*}$ & $1.932(0.306)^{*}$ & $2.305(0.332)^{*}$ & $3.519(0.508) *$ \\
\hline ICC & 0.41 & 0.37 & 0.41 & 0.52 \\
\hline$* \mathrm{p}<0.001$ & & & & \\
\hline
\end{tabular}

The unconditional model (model 0) allows for the calculation of the ICC, which provides an estimate of the total variance in ESI prescription that is related to differences between clinicians rather than to differences in CANS response patterns. Calculated ICCs ranged from 0.37 for the behavior-focused treatment outcome to 0.52 for the substance use-focused treatment outcome. These variance proportions clearly indicate the need for multilevel analysis. 


\section{The fixed effects CANS predictors only model (Model 1).}

Table 4.16. Fixed CANS Predictors Only (Model 1) Coefficients

\begin{tabular}{lcccc}
\hline & & \multicolumn{2}{c}{ Outcome Variable } \\
& $\begin{array}{c}\text { Trauma- } \\
\text { Focused }\end{array}$ & $\begin{array}{c}\text { Behavior- } \\
\text { Focused }\end{array}$ & $\begin{array}{c}\text { Family- } \\
\text { Focused }\end{array}$ & $\begin{array}{c}\text { Substance Use- } \\
\text { Focused }\end{array}$ \\
\hline Intercept $\left(\gamma_{00}\right)$ & $-2.325(0.171)^{*}$ & $1.799(0.197)^{*}$ & $-2.587(0.209)^{*}$ & $-4.001(0.268)^{*}$ \\
Trauma Scale $\left(\gamma_{10}\right)$ & $0.155(0.014)^{*}$ & $0.062(0.015)^{*}$ & $-0.013(0.014)$ & $0.045(0.017)^{* *}$ \\
Behavior Scale $\left(\gamma_{20}\right)$ & $0.029(0.012)^{* *}$ & $0.065(0.017)^{*}$ & $0.072(0.013)^{*}$ & $0.028(0.019)$ \\
Family Scale $\left(\gamma_{30}\right)$ & $0.009(0.007)$ & $-0.004(0.009)$ & $0.005(0.008)$ & $0.005(0.009)$ \\
Substance Scale $\left(\gamma_{40}\right)$ & $-0.042(0.018)^{* *}$ & $0.027(0.027)$ & $-0.016(0.018)$ & $0.089(0.026)^{*}$ \\
Variance $\left(u_{0 \mathrm{j}}\right)$ & $2.205(0.301)^{*}$ & $1.993(0.326)^{*}$ & $2.035(0.310)^{*}$ & $3.368(0.506)^{*}$ \\
$* p<0.001 * * p<0.05$ & & & & \\
\hline
\end{tabular}

The four CANS-derived predictor variables are introduced in model 1 as fixed (i.e., no clinician-level error terms are defined). Regression coefficients and standard errors are presented in Table 4.16. In the trauma model, three of the four predictors were significantly associated with the prescription of a trauma-focused treatment: trauma scale $(t=10.979, p<0.001)$, emotional/behavioral scale $(t=2.369, p=0.018)$, and substance use scale $(t=-2.362, p=0.018)$. In the behavior model, two of the four predictors were significantly associated with the prescription of a behavior-focused treatment: trauma scale $(t=4.142, p<0.001)$ and emotional/behavioral scale $(t=3.715, p<0.001)$. The emotional/behavioral scale $(t=5.507, p<0.001)$ was the only predictor significantly associated with the prescription of a family-focused treatment recommendation. Two predictors - trauma scale $(t=2.671, p=0.008)$ and substance use scale $(t=3.386, p=$ 0.001) - emerged as significantly predictive of a prescribed substance use-focused ESI. 
The random effects CANS predictors only model (Model 2).

Table 4.17. Random Effects CANS Predictors Only (Model 2) Level-2 Variance Estimates

\begin{tabular}{lcccc}
\hline & Trauma- & \multicolumn{2}{c}{ Outcome Variable } & \\
& Focused & $\begin{array}{c}\text { Behavior- } \\
\text { Focused }\end{array}$ & $\begin{array}{c}\text { Family- } \\
\text { Focused }\end{array}$ & $\begin{array}{c}\text { Substance Use- } \\
\text { Focused }\end{array}$ \\
\hline Trauma Scale $\left(u_{1 j}\right)$ & $0.003(0.002)$ & $0.000^{\mathrm{a}}$ & $0.001(0.002)$ & $0.000^{\mathrm{a}}$ \\
Behavior Scale $\left(u_{2 j}\right)$ & $0.001(0.001)$ & $0.005(0.003)$ & $0.000(0.001)$ & $0.000^{\mathrm{a}}$ \\
Family Scale $\left(u_{3 j}\right)$ & $0.001(0.000)$ & $0.000(0.000)$ & $0.000(0.000)$ & $0.000(0.001)$ \\
Substance Scale $\left(u_{4 j}\right)$ & $0.000^{\mathrm{a}}$ & $0.003(0.008)$ & $0.000^{\mathrm{a}}$ & $0.002(0.007)$ \\
a Redundant parameter & & & & \\
\hline
\end{tabular}

A series of models (model 2) were produced in which the slopes for each predictor were permitted to vary randomly across level-2 units (i.e., clinicians). One at a time, all predictor variables were included in all four different treatment outcome models - both those that were identified as statistically significant in model 1 and those for which no significant association with the outcome variable were observed. As summarized in Table 4.17, there were no significant random effects of any of the predictors across any of the outcome variables. This suggests that while there is substantial variation between clinician-level groups (as indicated by the ICC), it is not attributable to differences in the predictor variables among level-2 units. 


\section{The fixed effects CANS \& child demographics predictors model (Model 3).}

Table 4.18. Fixed CANS \& Child Demographic Predictors Model (Model 3) Coefficients

\begin{tabular}{|c|c|c|c|c|}
\hline & \multicolumn{4}{|c|}{ Outcome Variable } \\
\hline & $\begin{array}{l}\text { Trauma- } \\
\text { Focused }\end{array}$ & $\begin{array}{l}\text { Behavior- } \\
\text { Focused }\end{array}$ & $\begin{array}{l}\text { Family- } \\
\text { Focused }\end{array}$ & $\begin{array}{l}\text { Substance Use- } \\
\text { Focused }\end{array}$ \\
\hline Intercept $\left(\gamma_{00}\right)$ & $-2.072(0.289)^{*}$ & $-0.191(0.382)$ & $-2.442(0.335)^{*}$ & $-5.469(0.575)^{*}$ \\
\hline Trauma Scale $\left(\gamma_{10}\right)$ & $0.150(0.014)^{*}$ & $0.070(0.017)^{*}$ & $-0.013(0.014)$ & $0.049(0.017)^{* *}$ \\
\hline Behavior Scale $\left(\gamma_{20}\right)$ & $0.033(0.012)^{* *}$ & $0.063(0.018)^{*}$ & $0.072(0.013)^{*}$ & $0.028(0.018)$ \\
\hline Family Scale $\left(\gamma_{30}\right)$ & $0.008(0.007)$ & $0.004(0.010)$ & $0.005(0.008)$ & $0.008(0.009)$ \\
\hline Substance Scale $\left(\gamma_{40}\right)$ & $-0.030(0.018)$ & $-0.041(0.029)$ & $-0.013(0.019)$ & $0.057(0.029)^{* *}$ \\
\hline Age $\left(\gamma_{50}\right)$ & $-0.039(0.019)^{* *}$ & $0.167(0.030)^{*}$ & $-0.011(0.021)$ & $0.117(0.039)^{* *}$ \\
\hline Gender: Female $\left(\gamma_{60}\right)$ & $0.379(0.134)^{* *}$ & $0.163(0.160)$ & $-0.033(0.136)$ & $-0.112(0.234)$ \\
\hline Variance $\left(u_{0 \mathrm{j}}\right)$ & $2.230(0.304)^{*}$ & $2.134(0.351)^{*}$ & $2.036(0.311)^{*}$ & $3.526(0.528)^{*}$ \\
\hline$* p<0.001 \quad * * p<0.05$ & & & & \\
\hline
\end{tabular}

In model 3 (Table 4.18) two child-level demographic predictors are added: age and gender. The inclusion of these predictors was informed by the group differences identified during the earlier descriptive analysis. The reference group for gender is male. With the inclusion of the additional predictors, the substance scale was no longer a statistically significant predictor of trauma-focused ESI, though both age $(t=-2.004, p=$ $0.041)$ and gender $(t=2.831, p<0.005)$ were significantly associated with a traumafocused treatment prescription. In relation to the behavior-focused outcome, the trauma scale and the emotional/behavioral scale remained significant as was the newly included age variable $(t=5.649, p<0.001)$. Neither of the child-level demographic variables were significantly associated with the prescription of a family-focused treatment. In addition to the two significant predictors from model 1 - trauma scale and substance use scale — age $(t=3.021, p=0.003)$ was also found to be significantly associated with the prescription of a substance use-focused ESI. 
Model evaluation and comparison.

Table 4.19. Evaluation and Comparison of Models

\begin{tabular}{lcccc}
\hline Model & $\sigma_{R}^{2}$ & $R_{1}^{2}$ & $R_{M Z}^{2}$ & $\phi$ \\
\hline Trauma & & & & \\
\hline Model 0 & 3.290 & -- & -- & $0.613^{*}$ \\
Model 1 & 2.754 & 0.163 & 0.166 & $0.657^{*}$ \\
Model 3 & 2.729 & 0.170 & 0.172 & $0.661^{*}$ \\
Behavior & & & & \\
\hline Model 0 & 3.290 & -- & -- & $0.308^{*}$ \\
Model 1 & 2.995 & 0.090 & 0.089 & $0.387^{*}$ \\
Model 3 & 2.815 & 0.144 & 0.140 & $0.437^{*}$ \\
Family & & & & \\
Model 0 & 3.290 & -- & -- & $0.384^{*}$ \\
Model 1 & 3.171 & 0.036 & 0.038 & $0.353^{*}$ \\
Model 3 & 3.170 & 0.036 & 0.038 & $0.353^{*}$ \\
Substance Use & & & & \\
Model 0 & 3.290 & -- & -- & $0.601^{*}$ \\
Model 1 & 3.119 & 0.052 & 0.053 & $0.631^{*}$ \\
Model 3 & 3.026 & 0.080 & 0.080 & $0.632^{*}$ \\
${ }^{*} p<0.001$ & & & & \\
\hline
\end{tabular}

To estimate the explained first-level (i.e., child-level) variance the models were first brought to the same scale via the calculation of a scale-correction factor. For all unconditional models, the first-level variance $\left(\sigma_{R}^{2}\right)$ has a distributional value of 3.29. For each subsequent model, the first-level variance was scaled by multiplying by the square of the calculated scale-correction factor. The proportion of explained first-level variance $\left(R_{1}^{2}\right)$ can then be calculated. Similarly, the McKelvey and Zavoina $\mathrm{R}^{2}\left(R_{M Z}^{2}\right)$ was calculated for each outcome variable for models 1 and 3.

In the trauma model, the unexplained level one variance was reduced by $17 \%$ from the unconditional model to the full model. Approximately $9 \%$ of the variance in the prescription of a behavior-focused ESI was predicted by the CANS-derived predictors; the proportion of explained variance increased to roughly $14 \%$ with the inclusion of the 
demographic predictors. Neither the inclusion of the CANS-derived nor the demographic variables contribute much explanation to the family-focused treatment model with all predictors only explaining 3-4\% of the variance in the recommendation for some form of family therapy. The inclusion of CANS-derived predictors reduced the unexplained variance related to substance use-focused treatment by about $5 \%$ with an additional $3 \%$ of variance explained when the demographic variables were included.

To examine each model's classification accuracy, the dichotomous predicted and observed outcome group classifications were cross-tabulated and the phi coefficient $(\phi)$ calculated. The final trauma $(\Phi=0.661, p<0.001)$ and substance use $(\Phi=0.632, p<$ 0.001) models evidenced a strong positive correlation between predicted and observed outcomes with modest but progressive improvement across model iterations. The behavioral model improves from a weak predicted/observed outcome correlation in the unconditional model $(\Phi=0.308, p<0.001)$ to a moderately strong correlation $(\Phi=$ $0.437, p<0.001)$ in the final model. The unconditional family-focused model has a moderately weak predicted/observed outcome correlation $(\Phi=0.384, p<0.001)$; the introduction of predictors detracts from, rather than enhances, the model's classification accuracy. 


\section{Model interpretation.}

Table 4.20. Standardized Regression Coefficients - Model 1 \& Model 3

\begin{tabular}{lcccc}
\hline & \multicolumn{2}{c}{ Outcome Variable } \\
& $\begin{array}{c}\text { Trauma- } \\
\text { Focused }\end{array}$ & $\begin{array}{c}\text { Behavior- } \\
\text { Focused }\end{array}$ & $\begin{array}{c}\text { Family- } \\
\text { Focused }\end{array}$ & $\begin{array}{c}\text { Substance Use- } \\
\text { Focused }\end{array}$ \\
\hline Model 1 & & & & \\
Trauma Scale & $0.35^{*}$ & $0.15^{*}$ & -0.03 & $0.10^{*}$ \\
Behavioral Scale & $0.08^{*}$ & $0.18^{*}$ & $0.21^{*}$ & 0.07 \\
Family Scale & 0.04 & -0.02 & 0.03 & 0.02 \\
Substance Scale & $-0.07^{*}$ & 0.04 & -0.03 & $0.13^{*}$ \\
Model 3 & & & & \\
Trauma Scale & $0.34^{*}$ & $0.16^{*}$ & -0.03 & $0.11^{*}$ \\
Behavioral Scale & $0.09^{*}$ & $0.17^{*}$ & $0.21^{*}$ & 0.07 \\
Family Scale & 0.04 & 0.02 & 0.03 & 0.04 \\
Substance Scale & -0.05 & -0.07 & -0.02 & $0.08^{*}$ \\
Age & $-0.06^{*}$ & $0.24^{*}$ & -0.02 & $0.16^{*}$ \\
Gender: Male & $0.07^{*}$ & 0.03 & -0.01 & -0.02 \\
\hline
\end{tabular}

To assess the relative contribution of individual predictors, regression coefficients were standardized to eliminate differences in the scales of the explanatory variables (see Table 3.2). As hypothesized, the CANS-derive trauma scale was the most powerful predictor of a trauma-focused treatment prescription in both model 1 and model 3. Likewise, the CANS-derived emotional/behavior scale was the most important predictor of a behavior-focused ESI in the CANS predictors only model, though age was even more strongly associated with a behavior-focused treatment prescription when the demographic variables were entered. The emotional/behavioral scale also emerged as the most important predictor of a family-focused treatment prescription; however, the poor explanatory power and classification accuracy of this model calls into question the merit of any additional interpretation of this model. In the CANS-derived predictor only model, the substance use scale was the most powerful predictor of a substance use-focused 
treatment recommendation. After demographic variables were added to the model, the trauma scale's impact became more pronounced.

Table 4.21. Odds Ratios - Model 3

\begin{tabular}{lcccc}
\hline & & \multicolumn{2}{c}{ Outcome Variable } \\
& $\begin{array}{c}\text { Trauma- } \\
\text { Focused }\end{array}$ & $\begin{array}{c}\text { Behavior- } \\
\text { Focused }\end{array}$ & $\begin{array}{c}\text { Family- } \\
\text { Focused }\end{array}$ & $\begin{array}{c}\text { Substance Use- } \\
\text { Focused }\end{array}$ \\
\hline Model 3 & & & & \\
Trauma Scale & $1.162^{*}$ & $1.073^{*}$ & 0.878 & $1.050^{*}$ \\
Behavioral Scale & $1.034^{*}$ & $1.065^{*}$ & $1.075^{*}$ & 1.028 \\
Family Scale & 1.008 & 1.004 & 1.005 & 1.008 \\
Substance Scale & 0.970 & 0.066 & 0.987 & $1.058^{*}$ \\
Age & $0.962^{*}$ & $1.182^{*}$ & 0.989 & $1.124^{*}$ \\
Gender: Female & $1.461^{*}$ & 1.177 & 0.968 & 0.894 \\
\hline
\end{tabular}

Unstandardized regression coefficients (i.e., estimated log odds) were converted to an odds ratio through exponentiation. For every one-unit increase on the trauma exposure scale, a child's probability of being prescribed a trauma-focused ESI increased by about $16 \%$ while every one-unit increase on the emotional/behavior scale increased the likelihood by more than $3 \%$. As a child's age increased, his or her probability of being prescribed a trauma-focused treatment decreased slightly, with each additional year of age reducing the likelihood by about $4 \%$. Finally, females were almost 1.5 times as likely as males to be prescribed a trauma-focused ESI when all other predictors are held constant.

The odds of being prescribed a behavior-focused treatment increased by about $7 \%$ with each one-unit increase in both the trauma exposure score $(\mathrm{OR}=1.073)$ and the emotional behavioral score $(\mathrm{OR}=1.065)$. When controlling for CANS responses and gender, older children were more likely than younger children to be prescribed a 
behavior-focused ESI with each one year increase in age corresponding with a more than $18 \%$ increase in probability of being recommended for behavior-focused treatment.

A one-unit increase on the emotional/behavioral scale corresponded with about an $8 \%$ increase in the probability of a family-focused treatment recommendation. However, as noted previously, the family-focused treatment model performed extremely poorly in terms of both prediction and classification suggesting that none of the included predictors are of much practical significance.

The probability of being prescribed a substance-use focused ESI increased by $5 \%$ for each one-unit increase on the trauma exposure scale and by almost $6 \%$ for each oneunit increase on the substance use scale. Additionally, when other predictors were held constant, a one-year increase in age corresponded with about a $12 \%$ increase in the likelihood of being recommended substance-use focused treatment. 


\section{CHAPTER 5:}

\section{DISCUSSION, LIMITATIONS, AND CONCLUSIONS}

\section{Discussion}

While prior research has clearly demonstrated that children in out of home care have extensive mental and behavioral health needs (Burns et al., 2004; Lehmann, Havik, Havik, \& Heiervang, 2013; Oswald, Heil, \& Goldbeck, 2010; Stahmer et al., 2005) considerably less is known about the service delivery environment intended to address these needs (Baker, Schneiderman, \& Licandro, 2017). Encompassing more than 2300 children, 9 service regions, 51 child-serving agencies, and 381 clinicians, the present study's findings have provided insight of considerable breadth into a recently redesigned child welfare service landscape. For the first time, patterns of treatment prescription across an entire service system were analyzed and described. Using data derived from the CANS functional assessment and CANS assessment report, the author sought to determine if there is a predictable relationship between particular assessment responses and the type of ESI prescribed. In preparation for the multilevel modeling employed to analyze the nature of this relationship, extensive descriptive analyses were conducted and a number of secondary research questions were explored.

The discussion of study findings begins by offering an overview of what has been learned about treatment prescription within this service system. The implication of these findings for practitioners, administrators, and educators are considered as well as areas in need of additional research attention. Next, a brief overview of descriptive CANS 
findings are outlined before moving into a more detailed discussion centered around each of the four modeled assessment dimensions - trauma exposure, emotional/behavioral problems, family functioning, and substance use. For each dimension, the prior research, present implications, and future research needs are addressed. Following this discussion narrative, the study's limitations are identified. The present chapter concludes by integrating empirical findings into the underlying theoretical framework and summarizing the key findings within this conceptual context.

\section{Treatment Prescription}

Dramatically different treatment prescription patterns were observed among the clinicians who completed one or more initial CANS assessments. At the most homogenous extreme, it appears that some clinicians' therapeutic approach is driven by a single modality irrespective of assessed client needs and strengths. At the opposite end of the spectrum, a subset of clinicians seem to embrace an extremely eclectic treatment philosophy assigning as many as seven different modalities to a single case. It is hard to conceive of either of these extremes being particularly conducive to assessment-driven treatment decision making. However, on average, clinicians in the sample completed about six CANS assessments and prescribed between two and three different modalities. This indicates that there is a moderate degree of variation in treatment recommendation across the typical clinician's assessment caseload and underscores the importance of research seeking to better understand the mechanisms underlying this variation.

Frequency counts of the specific ESIs prescribed within the system offer a broad overview of the treatments most often recommended across all cases, clinicians, and 
agencies. These counts evidence a service system dominated by a single treatment modality - cognitive-behavioral therapy. Almost three-fourths of all cases in the sample had been prescribed CBT and the vast majority of clinicians and agencies were associated with at least one case for which the modality had been prescribed. This finding is not altogether unexpected. More than a decade ago, it was recognized that CBT was becoming the "majority orientation" of practicing therapists (Gaudiano, 2008). Across psychiatry, psychology, and social work programs, training in CBT is offered more frequently than any other ESI (Weissman et al., 2006). In a national survey of licensed clinical social workers, $72.9 \%$ of respondents reported using CBT in their practice-more than any other type of therapeutic intervention (Pignotti \& Thyer, 2009). Clearly, the preeminence of CBT is not limited to this particular service system.

In and of itself, the over-representation of CBT may not be especially troubling; the modality undoubtedly has considerable merit, particularly in terms of empirical support. Hofmann and colleagues (2012) reviewed 106 meta-analyses examining the efficacy of CBT and concluded that the evidence-base of CBT is "enormous" and "very strong" (p. 436). Additionally, it is considered to be a cost-effective treatment for a number of pediatric mental health conditions (Dickerson, Lynch, Leo, DeBar, Pearson, \& Clarke, 2018; Haby, Tonge, Littlefield, Carter, \& Vos, 2004; van Steensel, Dirksen, \& Bogels, 2014).

Nonetheless, CBT must not be viewed as a one-size-fits-all treatment approach. For example, evidence suggests that it may not be more effective than other forms of psychotherapy for treating violent behavior in youth (Ozabaci, 2011), adolescent substance use (Hofmann et al., 2012), or victims of childhood sexual abuse (Macdonald 
et al., 2012). Moreover, while CBT's efficacy has been evaluated in relation to pediatric populations, there is gap in the research knowledge regarding its use with other subgroups such as minorities and low-income populations (Hofmann et al., 2012). Thus, while the frequency of CBT prescription observed in the current analysis may not be intrinsically concerning, indications that it may be being prescribed indiscriminately (as suggested by the absence of any meaningful association between some assessed needs and the prescribed treatment) or at the expense of other, potentially more effective treatment approaches (as suggested by infrequency of some ESI prescriptions across the entire sample) do signal a troubling practice trend.

While the treatment prescription findings are limited to those children for whom a KY CANS 5+ functional assessment had been completed and thus may not exhaustively capture all services recommended by a particular clinician or agency, they do offer novel insight into the state's OOHC service system. From this broad vantage point, one observes a system with limited treatment variation - at least in so far as what is being recommended at the time of the initial CANS. This finding has direct implications at the clinician, agency, and system level.

In relation to clinicians, the relative homogeneity of treatment prescriptions may indicate a need for broadened therapeutic expertise among treatment providers within this service system. A number of interventions that are relevant to child welfare populations and supported by empirical evidence are currently only being prescribed by an extremely limited subset of practitioners. Of the 17 treatment modalities listed on the CANS assessment report, 12 were prescribed by fewer than $10 \%$ of the sampled clinicians; only CBT and TF-CBT were prescribed by more than half. 
Recent research evidence suggests that service providers themselves recognize the need for broadened expertise. Thompson \& Colvin (2018) conducted focus groups with therapeutic service providers working with the Florida child welfare system to identify perceived gaps in knowledge and skills. Providers consistently expressed a need "to be competent in a range of specialized areas" and to develop "greater expertise across a range of therapeutic modalities" (pp. 63-64). The data suggest that providers in Kentucky's service system might voice a similar sentiment.

While this finding has obvious implications in terms of enhancing training and on-the-job learning opportunities for those clinicians currently practicing in the field, it also speaks to graduate-level education programs. As noted previously, CBT is the primary intervention taught to students in psychiatry, psychology, and social work; increasing exposure to and training in other ESIs may provide novice clinicians with a broader repertoire of clinical skills.

At an agency level, this finding may signal an opportunity for service specialization. Over the past several decades, private agencies have increasingly been contracted with to provide therapeutic services to child welfare populations (Thompson \& Colvin, 2018). With the ongoing shift toward an EBP orientation, such contracts often function as "de facto policies guiding EBI [evidence-based intervention] implementation" and organizational administrators are still learning to navigate this new procurement and contracting landscape (Willging, Gunderson, Green, Jaramillo, Garrison, Ehrhart, \& Aarons, 2018). Nationally, child welfare administrators have voiced a need for agencies that can provide specialized services (e.g., family therapy, school-based interventions) and better meet the needs of specific populations (e.g., victims of sexual abuse, children 
with developmental disorders) (Kolko, Herschell, Costello, \& Kolko, 2009). Agencies that are prepared to provide a range of specialized, empirically supported interventions or offer ESIs that are not available elsewhere in the service system may have an advantageous position in the competitive service landscape.

While these findings have provided a previously unavailable overview of the treatments being recommended to children in this $\mathrm{OOHC}$ service system, they also highlight the need for additional research. Given its clear prominence, analyses focused exclusively on the prescription and use of CBT within this service system may provide actionable information that is directly relevant for a sizeable proportion of the $\mathrm{OOHC}$ population. While the present analysis has clearly demonstrated that CBT is the most frequently prescribed treatment modality at the case, clinician, and agency level, it is far less clear the degree to which the actual therapeutic sessions accurately reflect this almost ubiquitous label. Creed and colleagues (2016) found that a therapist's self-reported identification as operating from a cognitive-behavioral theoretical orientation was not a valid predictor of demonstrated CBT skills as rated by expert observers. That is to say, therapists who labeled themselves as "CBT therapists" were no more competent at actually providing CBT than those who did not endorse such a label. It is entirely possible that a similar phenomenon — inaccurate treatment labeling —is producing inflated frequency counts that are misleading in relation to the number of cases that are receiving bona fide CBT therapy.

Fidelity assessments could provide valuable insight into this issue. For instance, the Cognitive Therapy Rating Scale (CTRS; Young \& Beck, 1980) provides a validated measure of CBT fidelity and offers a cutoff score to distinguish between CBT 
competency and non-competency. While such an undertaking would require a substantial research investment, even a relatively modest but random sample of service providers could establish estimated rates of CBT fidelity within this service system. This information could subsequently inform service enhancement initiatives. For instance, if the data suggest that the CBT labels are largely accurate (i.e., CBT is being delivered with fidelity), efforts could shift toward identifying those cases for whom the modality is most effective - and perhaps even more importantly, those cases for whom a different approach is indicated. By contrast, if it appears that CBT has become a generic label applied to a broad range of otherwise ill-defined psychotherapeutic techniques (i.e., CBT is not being delivered with fidelity), efforts to improve treatment decision making would be premature.

\section{CANS Functional Assessment}

In terms of both clinicians and agencies, there is considerable variation in the degree of experience administering the KY CANS 5+ assessment to children in OOHC. While more than a quarter of included clinicians had only completed one functional assessment at the time of the present analysis, some clinicians had completed fifty or more. Likewise, more than half of all assessments included in the sample were associated with just five child-serving agencies while about a third of the included agencies were associated with 10 or fewer completed assessments.

This finding is important for administrators, trainers, and evaluators to note. It suggests that even as functional assessment becomes more embedded in the practice culture and the total number of CANS completed within the service system multiplies, 
much of the direct hands-on experience with the instrument may be concentrated in a limited subset of clinicians and agencies. This underscores the need to provide stratified training opportunities such that clinicians with extensive CANS experience can be challenged to continue refining their use of the instrument (e.g., incorporating assessment findings into case conceptualizations and treatment selection) while still offering introductory or fundamental training opportunities for those with less experience.

Descriptive findings from the CANS instrument provide insight into the prior experiences, clinical needs, and behavioral patterns present in the population of children residing in the state's OOHC service system. Because all the assessments included in the analysis were the initial CANS, these findings reflect the youth's state shortly after entry into care.

Interestingly, the child strengths domain was assessed to be the most problematic at the time of the initial CANS with an average standardized score more than twice that of the next highest domain score. This finding is particularly concerning in light of research indicating that child strengths can significantly buffer the negative impacts of trauma (Griffin, Martinovich, Gawron, \& Lyons, 2009; Kisiel et al., 2017). It possible that the magnitude of the child strengths domain score in relation to other domain scores reflects differences in the way that strengths and needs are assessed. Perhaps assessing clinicians — still in the initial stage of the therapeutic relationship — feel more confident documenting the absence of strengths than they do the presence of risk behaviors or other indicators of psychosocial need. Ongoing evaluation provides some empirical support to this speculation. Preliminary longitudinal analysis by the Project SAFESPACE evaluation team have demonstrated substantial reductions in the child strengths domain 
score from the initial CANS to the first 90 day reassessment. This may indicate that previously overlooked child strengths are more readily identified as the clinician develops greater familiarity with the youth. There is limited research exploring the strengths of child welfare populations in general (Kisiel et al., 2017) and to the present author's knowledge no studies have examined the trajectory of assessed child strengths over the course of treatment. As such, this is an area that warrants continued investigation. The data now being collected through the system-wide implementation of the CANS functional assessment is highly amenable to such an analysis.

\section{Trauma Exposure and Trauma-Related Symptoms}

The trauma experiences module was the most frequently triggered of the eight contingency assessment modules included in the KY CANS 5+. More than half $(55.3 \%)$ of the sample was assessed to have experienced at least one type of trauma over the course of their lifetime. Surprisingly, this is slightly lower than the proportion of children in the general population who report exposure to at least one traumatic experience (68.2\%; Copeland, Keeler, Angold, \& Costello, 2007). While a number of previous studies have explored trauma experiences among various child welfare-involved populations (e.g., Briggs, Greeson, Layne, Fairbank, Knoverek, \& Pynoos, 2012; Dorsey, Burns, Southerland, Cox, Wagner, \& Farmer, 2012; Salazar, Keller, Gowen, \& Courtney, 2013), Griffin and colleagues' (2011) research is notable for using the same instrument to measure trauma (i.e., the CANS trauma experiences module) and a similar sampling frame (i.e., children entering $\mathrm{OOHC}$ in Illinois). They found that the proportion of youth suspected (i.e., a CANS item response greater than one) to have experienced at least one 
type of trauma was more than $95 \%$ - more than one and a half times that of the present study.

Descriptive findings indicate that among those children with any assessed trauma history, multiple types of traumatic experiences are the norm. Less than one fifth of these children had been exposed to a single type of traumatic experience with the average number of unique trauma experiences among this subgroup being more than three. This is consistent with prior research examining trauma exposure among at-risk or child welfare involved youth (Briggs et al., 2013; Griffin et al., 2011). Likewise, the most commonly experienced trauma types assessed in the present sample — neglect, family violence, emotional/verbal abuse, and physical abuse — are also largely consistent with findings from other studies investigating child welfare populations (Griffin et al., 2011; Kisiel et al., 2009).

Of note, a nontrivial proportion of the sample $(\sim 11 \%)$ was assessed to have experienced five or more types of traumatic experiences up to a maximum of 11 unique exposures. Given the robust research indicating a dose-response effect of increased adverse childhood experiences on the presence of such negative outcomes as increased risk behaviors (e.g., excessive drinking), early onset chronic disease (e.g., diabetes), and poor mental health (Chang, Jiang, Mkandawire, \& Shen, 2019; Sonu, Post, \& Feinglass, 2019), it is especially important that these children receive timely, trauma-focused ESIs. Child welfare administrators may wish to establish formal protocols for the rapid identification and careful outcome monitoring for these highly trauma-exposed cases. Proactively providing these children with intensive trauma-related services at the outset of their OOHC episode may reduce the risks of poor service outcomes that are associated 
with complex traumatization such as placement disruption (Weiner, Leon, \& Stiehl, 2011) or psychiatric hospitalizations (Kisiel et al., 2014). Likewise, additional research should focus specifically on the treatment trajectories of this particularly vulnerable subgroup to identify those placement settings wherein these youth are most successful.

The proportion of youth endorsing at least one trauma-related symptom was roughly comparable across both the Griffin et al. (2011) study ( 38\%) and the present study $(\sim 45 \%)$. By comparison, only $13.4 \%$ of trauma-exposed children in a general population sample evidenced any signs of trauma related symptoms (Copeland et al., 2007). The relative infrequency of dissociative symptoms compared to other traumatic stress symptoms is also consistent with prior research (Griffin et al., 2011; Kisiel et al., 2009).

In summary, present findings regarding the assessed trauma experiences and trauma-related symptoms are largely consistent with similar studies in all but one regard - the number of children assessed to have experienced at least one type of trauma exposure. This finding is difficult to interpret; taken at face value, it would suggest that Kentucky's OOHC population has been exposed to substantially fewer trauma experiences than not only a comparable population from a neighboring state but also children in the general population yet exhibits trauma symptoms at a higher rate than both. An alternative explanation for this unexpected finding entails differences in the CANS administration process between states. Both Griffin and colleagues (2011) and Kisiel and colleagues (2009) analyzed CANS assessments completed by the Illinois Department of Children and Family Services (DCFS) while in the present study the assessments were completed by contracted behavioral health service providers. It is 
plausible that child protection workers are more sensitive to and knowledgeable of the traumatic experiences of children entering care, including those that occurred much earlier in the case history, while behavioral health clinicians are better positioned to recognize current trauma-related symptoms than they are historical trauma exposure. Moreover, in the KY CANS 5+ the trauma experiences module must be triggered while the Illinois version of the CANS includes these items in all assessments. As such, a child entering $\mathrm{OOHC}$ in Kentucky who is not showing any indication of problems related to adjustment to trauma at the time of assessment will not have his or her previous trauma experiences assessed, even if they are in fact quite extensive. As this explanation is merely speculative, future research should investigate the accuracy of the KY CANS 5+ in capturing lifetime trauma exposure. Until such research has been conducted, CANSderived rates of trauma exposure should be recognized as potentially misleading.

Even with the potentially underestimated rates of trauma exposure, there is clearly an extensive need for trauma-informed intervention among this population. A multilevel logistic regression model was developed to determine if selected CANS response patterns predicted the prescription of a trauma-focused ESI. It was hypothesized that the traumarelated CANS items would be the most powerful predictor of trauma-focused treatment. Study findings support this hypothesis. The CANS-derived trauma and emotional/behavioral scales were both statistically significant predictors of a traumafocused treatment, with the trauma scale emerging as the most powerfully predictive. This is not unexpected as the emotional/behavioral problems scale and the trauma exposure scale were also the most strongly correlated amongst the CANS-derived predictors. In the final model, each one unit increase in the total emotional/behavioral 
needs score was associated with a 3\% greater likelihood in being assigned a traumafocused ESI while a one-unit increase on the trauma experiences scale increased the likelihood by more than $16 \%$. Overall, CANS-derived predictors accounted for about $17 \%$ of the variation in the prescription of a trauma-focused ESI.

The use of trauma-informed assessment practices to identify those children in need of evidence-based, trauma-focused treatment has been described as critically important for the well being of children in the child welfare system (Conradi et al., 2011). It is encouraging to see that current practices reflect this mandate (albeit to a limited degree) as evidenced by the significant predictive relationship between trauma-related CANS items and the prescription of a trauma-focused treatment. While it is clear that assessment responses are not the only - and perhaps not even the primary-factor in the decision to prescribe an ESI intended to address trauma, the evidence does suggest that they are a salient factor.

However, there is also ample room for improvement in the provision of assessment-driven, trauma-focused intervention. Only about one quarter of the total sample was prescribed a trauma-focused ESI. Note that this is a much smaller proportion of the total sample than that which was assessed to have a history of trauma exposure $(\sim 55 \%)$ or problems with trauma-related symptoms $(\sim 45 \%)$. The implication of these figures is clear: there is a sizeable portion of the $\mathrm{OOHC}$ population with identified trauma-related needs who are not currently receiving an evidence-based, trauma-focused treatment; this finding is consistent with prior research suggesting that traumatized youth in OOHC are undertreated (Ai, Foster, Pecora, Delaney, \& Rodriguez, 2013). 
The exploration of group differences in assessment responses indicated that, on average, female youth scored significantly higher on the CANS-derived trauma scale than did male youth. This finding is consistent with prior research suggesting that girls may exhibit more trauma-related difficulties than boys (Collin-Vezina, Coleman, Milne, Sell, \& Daigneault, 2011; Gauthier-Duchesne, Hebert, \& Daspe, 2017; Wamser-Nanney \& Cherry, 2018). However, even when assessment scores were held equal, clinicians were 1.5 times more likely to have prescribed trauma-focused treatments to female youth than to male youth. While the literature has focused more on gender differences in trauma exposure or trauma-related symptoms than on gender differences in trauma treatment, there is some available insight into possible explanations for this disparity.

Investigating trauma-related symptoms following sexual abuse, GauthierDuchesne and colleagues (2017) observed higher rates of externalizing symptoms in boys while girls were more likely to manifest traditional PTSD symptoms (i.e., re-experiencing the trauma, hyperarousal, avoidance behavior). If similar behavioral patterns were observed among trauma-exposed youth in the present sample, it is reasonable to speculate that the externalizing symptoms of traumatized boys might be more likely to result in a treatment recommendation focused on addressing behavioral concerns rather than trauma. Alternatively, research by Godinet, Li, and Berg (2014) suggests that trauma-exposed boys may experience a more rapid decline in symptoms than trauma-exposed girls. As the CANS trauma experiences items pertain to lifetime trauma exposure, it is possible that females continue to exhibit more readily recognized symptoms at the time of assessment than do males with a comparable trauma history, particularly in those cases where significant time has transpired since the trauma occurred. 
Much of the scholarly literature has focused on the identification of children with trauma-related difficulties through the use of screening and assessment (e.g., Conradi et al., 2011; Lang et al., 2017). While this is undoubtedly an important area of inquiry, the present findings suggest a need to also investigate those youth who have been assessed to have trauma-related needs but who have not been assigned a trauma-focused treatment. What child, clinician, or environmental factors influence clinical decision making in these cases? Present findings suggest that gender may be one salient factor, but a more comprehensive investigation is warranted.

\section{Emotional/Behavioral Needs}

Some degree of emotional/behavioral problems were almost ubiquitous in the study population with most (60.1\%) being rated at an actionable level of severity. Descriptive findings reveal a population fraught with anxiety, impulsivity, oppositional behavior, and depression. Unfortunately, these results are not unexpected; it is well recognized that children and youth in the $\mathrm{OOHC}$ service system exhibit more mental health problems than those in the general population (Parker, Jacobson, Pullmann, \& Kerns, 2019).

The prevalence of both mental health symptoms and trauma-related symptoms in this population implies that service providers must be prepared to address both psychiatric disorders and traumatization while recognizing that the two are neither synonymous nor mutually exclusive. As Griffin and colleagues (2011) note: "both a traumatized child and a child with bipolar disorder may have difficulty with regulating their emotions, even though the child with bipolar disorder never experienced a traumatic 
event and the traumatized child does not suffer from bipolar disorder" (p. 71). At the same time, exposure to childhood trauma and adversity is a potent risk factor for the development of mental health problems (Navalta, McGee, \& Underwood, 2018) suggesting a complex and intertwined symptom etiology for many of the children in this sample. It is precisely because of the complex constellations of mental health and traumarelated symptoms exhibited by youth in $\mathrm{OOHC}$ that the use of structured assessment to inform treatment decision making and ESI selection is of vital importance.

More than a quarter of the sample was associated with violent or aggressive behavior directed at others; just a slightly smaller proportion was associated with selfharming behaviors. The early identification and evidence-based intervention of children with dangerous behavioral patterns is particularly important as evidence suggests that aggressive behavior is among the most persistent over time among child welfare involved youth (McCrea, 2009). Moreover, research investigating the characteristics of "difficult to place" children indicates that destructive behavior - directed at self, others, or property_ is almost universal among this vulnerable subgroup (Armour \& Schwab, 2007). As might be expected, risk behaviors were positively correlated with assessed emotional/behavioral needs.

On average, males were assessed to have more emotional/behavioral problems than females. This is not particularly surprising given that the CANS child risk behaviors domain - which contributed slightly more than half of the items in this composite scaleis comprised primarily of externalizing problems (e.g., bullying, delinquent behaviors) and research indicates that boys generally exhibit more externalizing problems than girls (Bongers, Koot, van der Ende, \& Verhulst, 2003). Future research may benefit from a 
more nuanced exploration of emotional/behavioral problems that distinguishes between internalizing and externalizing symptoms.

A multilevel logistic regression model was used to investigate the relationship between CANS-derived predictors and the recommendation of an ESI targeting emotional or behavioral problems. As hypothesized, the emotional-behavioral scale emerged as the most robust predictor of behavior-focused treatment, though the trauma exposure scale was also significantly associated with the prescription of a behaviorfocused ESI. A one-unit increase on the emotional/behavioral problems scale increased the likelihood of behavior-focused treatment by about 7 percent after controlling for the child's age.

Just as the vast majority of the sample ( $\sim 94 \%)$ was assessed to have some degree of emotional/behavioral problems, the vast majority ( $90 \%)$ was assigned a behaviorfocused treatment. As such, there was not a great deal of variance in the behavior-focused treatment outcome to be explained. Altogether, CANS-derived predictors accounted for 9 percent of this variation in the prescription of a behavior-focused treatment. With the inclusion of child demographic predictors, the amount of explained child-level variance increased to more than 14 percent.

Findings indicate that when all other predictors are controlled, older children are more likely to be prescribed a behavior-focused treatment than younger children. Each additional year of age increases this probability by almost 18 percent. For example, if an 8-year-old and a 14-year-old were assessed to have identical emotional/behavioral concerns, the 14-year-old would be more than twice as likely to be assigned an ESI targeting behavior. 
There are reasons to be concerned about this finding. It has been estimated that as many as $40 \%$ of children under the age of six entering OOHC exhibit significant behavior problems yet less than one quarter receive any behavioral intervention (Stahmer et al., 2005). However, early childhood is likely the most opportune time to intervene. For example, research suggests that most children learn to regulate physically aggressive behavior very early in childhood and intervention should begin as early as aggressive tendencies are identified (Tremblay et al., 2004). Additional research should focus on the unique behavioral needs of young children in this service system as well as the system's current capacity for providing age-appropriate services.

As previously noted, among the four CANS-derived composite predictor scales, trauma exposure and emotional/behavioral problems were the most strongly correlated. Relatedly, trauma exposure emerged as a significant predictor of a behavior-focused treatment prescription just as emotional/behavioral problems emerged as a significant predictor of trauma-focused treatment. This finding is consistent with a large body of research linking childhood trauma to a host of disruptive emotional problems and maladaptive behaviors (Gerrity \& Folcarelli, 2008). An expanding body of research is investigating the impact of particular types of trauma on different behavioral outcomes. For example, there is compelling evidence that children who have experienced physical abuse engage in aggressive behaviors at a higher rate than children who have experienced other forms of maltreatment (Holmes, Yoon, Voith, Kobulsky, \& Steigerwald, 2015; Yoon, Tebben, \& Lee, 2017). Even more nuanced research may include child-level interaction effects. For instance, Kobulsky (2017) has found that physical abuse predicts early substance abuse in girls but not in boys. 
Clearly, the data now being collected through ongoing CANS assessment offers tremendous potential for academic researcher to conduct similar investigations across an entire state-level OOHC population. But perhaps even more importantly, the CANS assessment provides a platform for practitioners to make treatment decisions informed by recent research findings examining the link between specific trauma experiences and subsequent emotional/behavioral risks (such as those described above). While this may seem an overly ambitious objective given the widely acknowledged research-practice gap (e.g., Bledsoe-Mansori et al., 2017; Teater, 2017), there is some cause for optimism. Present findings suggest that practitioners' decisions to prescribe a behavior-focused ESI are already predicted, in part, by assessed trauma experiences; as such, building upon and expanding this already-existent practice may be feasible.

\section{Family Functioning}

Interestingly, only about three-fifths of the sample's caregivers were assessed to have any needs. Moreover, no single item in the CANS caregiver needs and strengths domain was assessed to be present in more than half the sample. That is to say, initial assessment data suggest that a substantial proportion ( 40\%) of caregivers had no recognized needs related to such areas as parenting knowledge, financial resources, mental health, substance use, partner violence, or residential stability. This finding stands in contrast to prior research indicating a much higher rate of need among child-welfare involved families. For example, in a sample of child welfare involved parents in Washington, Marcenko and colleagues (2009) found that $73 \%$ of parents had immediate financial needs and $87 \%$ reported experiencing at least one of four risk factors: domestic 
violence, sexual abuse, substance abuse, or mental health conditions. In a more recent study, Lee and Logan-Greene (2017) used latent class analysis to identify different classes of service needs among child welfare involved families; only about $20 \%$ of the sample was classified as "low needs" and even these families still reported some need for social services.

While it is not possible to conclusively determine that the assessment data related to caregiver needs is inaccurate without additional data collection and analysis, the most parsimonious explanation for these divergent findings suggests difficulty with the accurate assessment of family needs rather than their actual absence among the sample's caregivers. Other child-level CANS items support this conjecture. For example, descriptive findings indicate that almost $85 \%$ of cases have some degree of impaired family functioning and less than $15 \%$ of the sample had centerpiece strengths related to either nuclear or extended family relationships. Responses to these items suggest a more extensive prevalence of family-related problems than is indicated by those items assessed from the caregiver perspective.

Assuming that there is indeed some significant under-identification of caregiver needs, efforts to improve this aspect of the assessment process must be prioritized. Comprehensive family assessment has become a best practice in child welfare as they provide vital information about the family contexts affecting a child's safety, permanency, and wellbeing (Child and Family Services Reviews, 2017; Smithgall, JarpeRatner, Gnedko-Berry, \& Mason, 2015). If current assessment practices do not include an exhaustive attempt to accurately capture the needs of the child's caregiver, they can hardly qualify as comprehensive. 
While improving the accuracy of the caregiver needs assessment may entail an additional investment of clinician time and labor (e.g., contacting multiple family members, reviewing collateral documentation), it is a worthwhile and necessary investment. Federal-level child welfare policy has been growing increasingly familycentered in recent decades based on the premise that "supporting families and meeting families' needs can maximize gains for children" (Kilmer, Cook, Munsell, 2010, p. 333). Research evidence provides some support for this position. For example, substance abusing mothers who receive appropriately-matched services are more likely to reunify with children placed in OOHC (Choi \& Ryan, 2007). Adequately meeting the needs of families must begin with an accurate assessment of those needs. As Ingram and colleagues (2015) have noted: "careful assessment is the key to identification of risk and protective factors affecting the family and helps determine the most appropriate services" (p. 144).

Encouragingly, there is some indication that such "careful assessment" is occurring for many cases. Among only those cases for which any caregiver needs were identified, an average of eight different needs were assessed as requiring some degree of prevention or intervention. It would appear that when caregiver needs are assessed, they are assessed thoroughly. As such, efforts to improve comprehensive family assessment within this service system should first seek a better understanding of those cases for which caregiver assessment is altogether absent. Importantly, it should be determined if these apparently unassessed cases are primarily associated with family-level factors (e.g., some subset of families are particularly difficult to assess) or clinician-level factors (e.g., some subset of clinicians consistently do not complete caregiver assessments). 
Descriptive treatment prescription counts indicate that only about one-eighth of all children included in the sample were prescribed any form of family therapy. Moreover, the vast majority of those for whom family-focused treatment was recommended received a generic "family therapy" recommendation rather than a specific ESI (e.g., brief strategic family therapy, parent-child interaction therapy). No single family-focused ESI was prescribed to more than two percent of the total sample.

Multilevel logistic modeling indicates that CANS items have little to no predictive relationship with the prescription of a family-focused treatment. The final family-focused treatment outcome model explained less than 4 percent of the variance in the clinician's decision to prescribe a family-focused ESI. The hypothesis that CANS items related to family functioning would predict the prescription of a family-focused ESI was not supported. While scores on the emotional/behavioral scale were significantly associated with the decision to recommend family-focused treatment, the inclusion of CANS-related predictors actually degraded the model's classification accuracy.

Taken together, the descriptive findings and modeling outputs paint a troubling picture. Family functioning appears to be under-assessed. When it is assessed it appears to have little influence in the decision to recommend family-focused treatment. And when family-focused treatment is recommended, it is rarely an ESI.

The challenge of providing family centered service to child welfare involved families has been acknowledged for many decades (Cole, 1995); this challenge is no less pressing today. In their study of therapeutic service providers working with child welfare populations, Thompson and Colvin (2019) record a particularly illustrative participant quotation worth including verbatim: 
If you are not experienced in family therapy or didn't study family therapy in school or anything, it is like you are just kind of thrown in there and you got people screaming at each other and it is like...'I don't know what to do. I don't

have the proper tools. I don't know about the proper interventions (p. 64).

The present study's results provide a near perfect quantitative reflection of Thompson and Colvin's (2019) qualitative findings. Both imply a need to provide more extensive education, training, and supportive clinical supervision for clinicians tasked with assessing family's needs and delivering family therapy. There are also agency level implications; recent research suggests that efforts to implement family centered practices are most successful in agencies that are outward focused (i.e., focused on clients and their needs), innovative, and flexible (Ahn, Keyser, \& Hayward-Everson, 2016). Thus, agency administrators interested in improving family-focused service delivery must look not only to providing clinicians with additional training, skills, and support but also to establishing an organizational culture that is open to trying creative, consumer-minded service delivery strategies. The data clearly suggest that there is ample room for improvement in this area.

\section{Substance Use}

More than one quarter of the sample triggered the substance use module indicating some degree of concern related to alcohol or drug use. The prevalence of substance use-related issues in the present sample is considerably lower that of prior studies investigating child welfare involved youth (Fettes, Aarons, \& Green, 2013; Traube, James, Zhang, \& Landsverk, 2012; Vaughn, Ollie, McMillen, Scott, \& Munson, 
2007). However, this is primarily due to differences in sampling frames. Evidence clearly indicates that older age is a risk factor for substance abuse (Yampolskaya, Chuang, Walker, 2019). Most studies that have investigated substance use among child welfare involved populations have sampled older youth; the present sample includes children as young as five. Assessed substance use rates ( $45 \%)$ among those children older than 12 in the sample are much more comparable to previous findings.

In addition to the aged-based differences noted above, present findings indicate significant gender differences with males' substance use-related problems being assessed as more severe than females'. There were no significant differences in assessed substance use-related issues across racial/ethnicity categories. Previous findings have been mixed in relation to both gender and racial substance use patterns among child welfare-involved youth (Aarons et al., 2008; Cheng \& Lo, 2011; Traube et al., 2012).

Descriptive findings suggest that substance use tends to be habitual rather than experimental among those youth who were assessed to have problems related to drugs or alcohol. Three quarters of these youth had been using substances for at least one year and most had been abstinent for less than one month at the time of assessment. Research by Pittenger and colleagues (2018) indicates that youth who have already initiated substance use by the time of their first contact with the child welfare system are at the greatest risk for continued usage (compared to those who initiate substance use during or after their first contact). As current findings drew from the initial CANS assessment (which is completed within 30 days of the behavioral health referral), this risk factor may be particularly salient to consider. Those youth entering OOHC with an established history 
of substance use may benefit from more intensive or specialized substance abuse interventions.

The CANS functional assessment does not record the types of substances being used or distinguish between alcohol use and drug use. The screening instrumentcompleted prior to the referral to behavioral health and thus accessible to the assessing clinician - does provide some insight into the type of substances being used but is limited to only alcohol, marijuana, or "anything else." Moreover, the substance use portion of the screening instrument is only administered to children who are twelve-years-old or older. As such, there is presently no standardized, system-wide mechanism within the screening and assessment process to ascertain usage patterns related to specific substances. Given compelling evidence that there are different risk indicators and prevention strategies across substance types (Pittenger et al., 2018), administrators may wish to supplement existing screening/assessment measures to begin collecting this information. This would also facilitate a more nuanced analysis of substance-specific prevalence rates, usage trends, and treatment outcomes than is currently possible. For example, Pittenger and colleagues (2018) found that while the prevalence of marijuana use in a sample of child welfare involved youth was comparable to the general United States population, cocaine use was approximately four times more common among the child welfare sample. Determining whether this pattern holds true for the present sample would be challenging given the current absence of substance-specific measures.

Most youth who were assessed to have substance use related issues reported being negatively influenced by their peers, parents, and environments. Prior research has established peer, sibling, and parental substance involvement as a potent risk factor for 
substance use or dependence (Aarons et al., 2008; Yampolskaya et al., 2019). This is especially important to note in light of the fact that more than one quarter of the present sample's caregivers were assessed to have substance use related concerns. As such, an OOHC placement may provide an opportune setting for impactful intervention. Whereas there is some evidence that experiencing multiple out of home placements is associated with an increase in the severity of substance use (Aarons et al., 2008), OOHC itself may serve as a temporary protective factor. Both Cheng and Lo (2011) and Yampolskaya and colleagues (2019) observed a reduction in subsequent substance use following OOHC placement. Being placed in OOHC may in fact serve to "shield adolescents from unsafe environments and maladaptive influences" (Cheng \& Lo, 2011, p. 1671). This finding underscores the importance of providing timely, effective, and evidence-based substance use treatment in what may be the most important window of opportunity in many youth's life course.

While substance-using parents may be a recognized risk factor for youth substance involvement, parents and caregivers may also be valuable protective influences. Parental supervision and bonding/connectedness have been shown to reduce the likelihood of substance use (Cheng \& Lo, 2011; Hoffman \& Cerbone, 2002, Traube et al., 2012). Importantly, such positive parental influences are not limited to biological parents but extend to other caregivers such as foster parents (Cheng \& Lo, 2011). Unfortunately, descriptive results suggest that this potentially powerful protective factor is being underemployed in the current service environment. Those ESIs intended to decrease substance use in conjunction with increasing family/parental support (e.g., adolescent community reinforcement, brief strategic family therapy, wraparound) are 
among the least frequently prescribed modalities at the case, clinician, and agency levels. Future research should explore the reasons underlying the underutilization of these promising treatment approaches (e.g., lack of capacity, lack of family involvement).

Of the four focus-of-treatment outcomes modeled, substance use-focused treatment was the least frequently prescribed. Only 150 youth — about six percent of the sample — had been recommended an ESI intended to address substance use. This finding is alarming given the much larger proportion of the sample that was either recently abstinent $(\sim 15 \%)$ or still actively using substances $(\sim 5 \%)$ at the time of assessment. While research is sparse, it is generally understood that many substance using youth in the child welfare system either never receive substance abuse treatment or receive treatment approaches with a weak evidence base (Traube et al., 2012). Present findings support this claim.

CANS-derived predictors only accounted for about five percent of the variance in the prescription of a substance use-focused treatment; when age and gender when included in the model, the explained first-level variance increased to eight percent. This suggests that while the CANS assessment may have some degree of influence in substance use treatment decision making, it does appear to be one of the primary factors considered.

Of the four CANS-derived predictors included in the model, the substance use scale was the most powerful predictor for substance use focused treatment, providing support to the research hypothesis. However, after controlling for age and gender in the final model, the trauma exposure scale emerged as a more robust predictor than the substance use scale, though age was more predictive than either. For each one unit 
increase on the composite substance use scale, the likelihood of being prescribes a substance use-focused treatment increased by about six percent while each one year increase in age increased the probability by more than $12 \%$.

That trauma experiences were significantly associated with the recommendation for substance use-focused treatment is not altogether unexpected. The connection between childhood adversity and subsequent drug and alcohol use is well established. For example, Aarons and colleagues (2008) found that maltreatment history accounted for more variability in the severity of substance involvement than did demographic factors (e.g., race/ethnicity, household income). However, that trauma-related CANS items were a more robust predictor of substance use-focused treatment than assessed substance use itself is unexpected and difficult to explain. The trauma experiences scale and the substance use scale were the least strongly correlated of the four composite predictor scales so this finding is not a statistical artifact of collinearity.

One possible explanation for this finding is related to the inclusion of the trauma recovery and empowerment model (TREM) in both the composite substance use and the trauma focus-of-treatment outcome variables. TREM is an intervention designed to address the long-term cognitive, emotional, and social consequences of trauma including significant substance use problems (Fallot \& Harris, 2002). TREM accounted for almost one-third of all cases classified as receiving a substance use-focused treatment. If this modality is being used primarily to address trauma-related problems rather than substance use-related issues, the trauma experiences scale's relative strength in the prediction of a substance use-focused treatment would be inflated. Consequentially, this would mean that an even smaller proportion of cases are receiving treatment for the 
primary purpose of addressing substance involvement. Unfortunately, this possibility cannot be empirically verified given the available data. Future research exploring the relationship between assessment-derived predictors and specific ESIs may reduce some of the ambiguity arising from the use of composite outcome variables.

Altogether, these findings highlight considerable room for improvement in the treatment of substance use within the OOHC service environment. Perhaps most pressingly, it appears that a concerning number of youth with assessed drug and alcohol issues are not receiving any ESIs intended to address these problems. Improving the integration of assessment findings into the treatment decision making process may improve substance use intervention. However, the CANS assessment may also be of value for substance use prevention within this vulnerable population. Research has identified a number of specific factors that increase the probability of later substance involvement among OOHC populations including clinical level behavior problems, delinquency, dissociative symptoms, a history of sexual abuse, and exposure to domestic violence (Aarons et al., 2008; Goldstein et al., 2011; Traube et al., 2012; Yampolskaya et al., 2019). As all of these factors are assessed to some degree in the KY CANS 5+, clinicians can identify and proactively target those clients whose experiences and symptoms suggest an elevated risk for substance use, even if there is no current indication of substance involvement. Indeed, this primary prevention approach is likely the most effective. As Pittenger and colleagues (2018) have observed, "use begets use" (p. 93); as such, prevention efforts must be prioritized. 


\section{Limitations}

At the outset of the discussion of limitations it is important to highlight one aspect of the sampling frame that demands attention when interpreting study findings; namely, this sample included all children for whom initial functional assessment data was available. Theoretically, this should more-or-less reflect all children in OOHC who have screened in and, as a result, have been referred to a behavioral health service provider within this system of care. However, due to an assessment compliance rate of less than $50 \%$ thus far, the majority of children who should have been administered a CANS functional assessment have not. It is highly likely that there are non-random factors at the agency, clinician, and client level that impact CANS completion. While this limitation is unavoidable given the nature secondary data and is clearly noted as one of the inclusion criteria for this study, any generalization of these findings to this service system as a whole, or to other OOHC service systems, should be tempered by cognizance of this limitation.

It is important to note that this study reflects the treatment modality that children in OOHC were prescribed at time of assessment. It is not currently known the degree to which prescribed treatments align with delivered treatments in this service system although future analyses with these data will explore this question. Similarity, frequency counts of treatment modalities at the clinician or agency level reflect prescriptions as recorded on the CANS assessment report. These prescriptions should not be interpreted as necessarily indicative of any specialized training or certification in the identified modality or that the modality can be delivered with fidelity. As such, one should exercise caution in drawing firm conclusions about service capacity from these findings. 
These analyses were based solely on the child's initial CANS assessment. It is not yet known the degree to which treatment prescriptions are stable across a case's course of treatment. It is possible that the treatment recommendations tend to change after subsequent reassessments. Planned longitudinal analyses of these data will improve our understanding of any changes in treatment recommendation patterns over time. Currently, generalizations across time should be accepted as merely tentative.

As confirmed by the calculated intraclass correlation values, the analytic strategy of the present study (i.e. multilevel logistic regression) was an appropriate-and, in fact, necessary—approach given the data's clearly hierarchical structure. The multilevel approach was employed primarily to negate the independence of errors assumption that would have rendered traditional logistic regression untenable and made interpretation prone to error. The available secondary data did not include any second-level (i.e., clinician-level) variables. This was not problematic for the present study as the stated intent of this initial inquiry was to examine the association between CANS-derived predictors (which are first-level predictors) and treatment prescriptions. However, the lack of clinician-level variables in the data will be a substantial limitation for building upon these initial findings. Collecting clinician-level data and linking it to the available case-level data will likely require a substantial investment of time and expertise and may limit the ability to conduct additional system-wide analyses as recommended by the present author.

As with any form of multiple regression, the identification and selection of predictors (i.e., independent variables) entails some degree of subjectivity. Tabachnick and Fidell (2013) note that this decision may be guided by a theoretical framework, astute 
observations, or just a researcher's hunch; in the present case, each of these has had some measure of influence. There are other CANS-derived predictors that may have been viable candidates for inclusion in model building process (e.g., child strengths). Similarly, there are alternative options for the arrangement and selection of assessment items into the composite predictor scales (e.g., separating risk behaviors and emotional/behavioral needs into different predictor scales). Ultimately, following the advice of Tabachnick and Fidell (2013), the selection of predictors was driven by parsimony - that is, selecting the fewest necessary to predict the dependent variables. Future research may opt to include additional or modified CANS-derived predictor scales.

Three conditions must be satisfied to describe a relationship as causal: (1) the cause must precede the effect, (2) the cause must be related to the effect, and (3) there must be no other plausible explanation for the effect other than the cause (Shadish, Cook, $\&$ Campbell, 2002). In the design of Project SAFESPACE's service reconfiguration, the CANS functional assessment is intended to inform treatment planning and thus must precede it temporally. The online CANS Assessment Report, on which treatment recommendations are recorded, cannot be completed until the CANS functional assessment has been administered and the results entered. This study presupposes that this intended order is reflected in actual practice behavior - assessment first followed by treatment prescription. However, this cannot be confirmed by the research design and thus cannot satisfy the temporal condition of causal reasoning. Moreover, as a natural experiment using secondary data, very little manipulation or control is possible thus there is an inability to rule out other plausible explanations. The observed predictive 
association between response patterns on the CANS and ESI prescription must not be interpreted as definitively causal and alternative causal pathways should not be ruled out.

\section{Theoretical Integration and Conclusions}

The present study analyzed the relationship between the CANS functional assessment and the type of ESI prescribed to determine if CANS items related to trauma experiences, emotional/behavioral problems, family functioning, and substance use predicted an associated treatment prescription. Conceptually, this inquiry was contextualized within the EBP framework. As the "first step" and "foundation" of the EBP process (Grady \& Drisko, 2014; Simmons, 2011), structured assessments provide the clinician with information about the client's state and circumstances that can then guide every subsequent step of the EBP process. Despite its centrality to this process, the role of assessment in EBP has been almost entirely neglected in the research literature (Grady \& Drisko, 2014; Mash \& Hunsely, 2005). As such, the present findings add novel insight to an overlooked line of inquiry.

Within this broader EBP context, this study's empirical findings reflect a specific activity: treatment decision making — the "lynchpin" of EBP (Spring, 2008). The EBP model implies that treatment decisions should be guided by a number of factors including the client's state and circumstances (i.e., assessment findings), the client's preferences, the person of the practitioner, theory and research evidence, and professional expertise (Gilgun, 2005; Regehr et al., 2007). A number of theoretical models and conceptual frameworks have been developed to describe the process of decision making. Historically, EBP has been most closely aligned with the classical (i.e., rational choice) 
decision making tradition (van de Luitgaarden, 2009). However, the treatment decision making research literature provides little empirical support for this perspective. As such, naturalistic decision making has been suggested as an alternative framework for conceptualizing treatment decision making within EBP (e.g., Baker-Ericzen et al., 2015).

The pattern of modality prescription within this service system is suggestive of an underlying situation-action matching decision rule as conceptualized in NDM theory. Such a rule would imply that CBT is the standard treatment modality and is applied to any case for which it is deemed a "good enough" match; only when CBT is judged to be clearly insufficient are alternative modalities even considered. While this is not a theorytesting study and descriptive findings cannot confirm this conjecture, they are clearly more indicative of a matching rule than the concurrent choice rule posited by rational choice theory. Present findings in this regard are compelling enough to justify deeper inquiry. Future research, particularly of a qualitative nature, should explore the decision making processes of clinicians to provide additional insight into the decision rules employed in the treatment selection process.

The NDM approach recognizes that just because a source of information is available to the decision maker does not mean that this information is in fact used in the decision making process. As such, NDM-based models seek to identify what types of information actually influence decision making in the field setting. As noted above, there is a strong theoretical basis for concluding that assessments should inform subsequent treatment decisions, but is information gathered during the CANS assessment used to inform ESI selection in actual practice? This was the fundamental conceptual question underlying the present inquiry. 
Results of the multilevel logistic modeling suggest that clinicians in this service environment use assessment information to varying degrees when making treatment recommendations across different domains. Assessment-derived predictors accounted for more than $17 \%$ of the variance in the prescription of a trauma-focused treatment. While this falls slightly below the minimum threshold for "good" prediction (Hox et al., 2018), it does demonstrate a clear alignment between assessment findings and treatment recommendations. As the intensity of assessed trauma-related problems increased, so too did the probability of receiving an ESI intended to address trauma. Thus, it can be stated with reasonable confidence that assessment data appears influential in treatment decision making related to addressing traumatization. At the other extreme, none of the empirical findings provide any indication that assessment information plays a role in treatment decision making related to family functioning. In fact, the inclusion of assessmentderived predictors reduced the model's ability to accurately predict whether a case would or would not be prescribed a family-focused ESI. The other two domains of treatment decision making — emotional/behavioral problems and substance use — are a bit less clear. It should be noted that these two domains had the least variation in treatment prescription to explain. That is to say, the vast majority of the sample was prescribed a behaviorfocused treatment while only a fraction of the sample was prescribed a substance-focused treatment. Assessment-derived predictors accounted for less than ten percent of the limited variance in the prescription of a behavior-focused or substance use-focused ESI. At the same time, as assessed emotional/behavioral problems or substance use issues increased, so too did the probability of being prescribed a related ESI. One might tentatively conclude that assessment data appears to be a minor, but still salient, factor in 
the decision making process for these two treatment domains. Certainly, additional investigation is warranted before more definitive conclusions can be drawn.

In general, these conclusions are consistent with prior treatment decision making research suggesting that decisions are not directly attributable to any single factor (e.g., assessment-derived information) but are instead influenced by a number of considerations (Nelson \& Steele, 2008; Witteman \& Koele, 1999). They also acknowledge a differential use of assessment data that, when interpreted through the lens of NDM theory, suggest different process orientations across different domains of treatment decision making. Importantly, this implies that the question, "what factors influence treatment decision making?" may be too unidimensional in nature. A more viable research question may be, "what factors influence treatment decision making related to trauma (or substance use, or behavioral problems, etc.)?”.

Consistent with the NDM element of context-bound modeling, the primary aim of this study was to describe assessment patterns and associated treatment recommendations related to a particular population within a particular environment. Findings have direct and actionable implications for the practitioners, administrators, policymakers, educators, researchers, and other stakeholders associated with this OOHC service system. This is not to say that the present results do not have external relevance or advance the broader knowledge base. On the contrary, they contribute to the scholarly literature across a number of subject matters including the treatment needs and characteristics of youth in $\mathrm{OOHC}$, the use of structured assessments, and assessment-driven treatment planning. Nevertheless, the practical has been prioritized and no abstract, generalizable decision making models have been advanced. 
Just as it has informed the interpretation of current findings, NDM theory also offers a concrete next step in terms of building upon this line of research inquiry. Empirical-based prescription is an essential characteristic of the NDM framework. As such, description must precede prescription in terms of enhancing decision making performance. This study provides a comprehensive baseline description of structured assessment and treatment prescription across all clinicians who completed a CANS functional assessment. Future research should seek to compare these baseline findings with the treatment prescription patterns of clinicians with established expertise. Do expert clinicians prescribe a greater or lesser number of distinct modalities? Are expert clinicians' treatment recommendations more or less associated with assessment findings? As the treatment decision making practices and processes of expert clinicians are identified, they can serve as benchmarks for the system-wide enhancement of assessment-driven, evidence-based treatment recommendations.

Despite the lack of a definitive causal relationship (as detailed in the preceding limitations section), this study has offered valuable insight into the treatment decision making practices of clinicians in one OOHC service system and the degree to which these align with EBP. Importantly, this study has employed an underutilized approach for exploring EBP. Research examining the use of EBP in a practice environment tends to use self-report surveys (e.g., Stadnick et al., 2017), qualitative interviews and focus groups (e.g., Nelson et al., 2006), or knowledge and attitude scales (e.g., Aarons, 2004). While such investigations certainly have their merit, they also share a substantial limitation - if there is no professional or scholarly consensus regarding the definition of the term evidence-based practice (Rubin \& Parrish, 2007) and there are concerns that it is 
becoming little more than a catchphrase (Shlonsky \& Gibbs, 2004), how much is gained by asking practitioners if they engage in it or how they feel about it? Studies of this sort begin with a concept (EBP) and then must speculate as to the degree to which the conceptual is reflected in actual practice (see, for example, Tuten et al.'s, 2016, discussion of study limitations). This study approaches the question from precisely the opposite direction; drawing upon key NDM tenets, it begins with observable practice behaviors and speculates toward the conceptual. Certainly both vantage points have their respective blind spots, but the perspective presented herein has meaningfully contributed to the conceptual EBP knowledge base while simultaneously offering empirical findings with concrete implications for child welfare research and practice. 


\section{REFERENCES}

Aarons, G. (2004). Mental health provider attitudes toward adoption of evidence-based practice: The Evidence-Based Practice Attitude Scale (EBPAS). Mental Health Services Research, 6(2), 61-74. doi:10.1023/B:MHSR.0000024351.12294.65

Aarons, G. A., Hazen, A. L., Leslie, L. K., Hough, R. L., Monn, A. R., Connelly, C. D., ... Brown, S. A. (2008). Substance involvement among youths in child welfare: The role of common and unique risk factors. American Journal of Orthopsychiatry, 78(3), 340-349.

Abrefa-Gyan, T. (2016). Evidence-based practice: Attitude and knowledge of social workers across geographic regions. Journal Of Evidence-Informed Social Work, 13(6), 552-561. doi:10.1080/23761407.2015.1111826

Administration on Children, Youth, and Families. (2012). "Too many, too much, too young": Red flags on medications and troubled children. (2012). Reclaiming Children and Youth, 21(2), 59-61.

Ai, A. L., Foster, L. J. J., Pecora, P. J., Delaney, N., \& Rodriguez, W. (2013). Reshaping child welfare's response to trauma: Assessment, evidence-based intervention, and new research perspectives. Research on Social Work Practice, 23(6), 651-668. https://doi-org.echo.louisville.edu/10.1177/1049731513491835

Ahn, H., Keyser, D., \& Hayward-Everson, R. A. (2016). A multi-level analysis of individual and agency effects on implementation of family-centered practice in child welfare. Children \& Youth Services Review, 69, 11-18.

Andershed, A. \& Andershed, H. (2016). Improving evidence-based social work practice with youths exhibiting conduct problems through structured assessment.

European Journal of Social Work, 19(6), 887-900.

Anderson, R. L. (2008). Finding the balance in evolving service sectors for youth with co-occurring disorders: Measurement and policy implications. Residential Treatment for Children \& Youth, 24(3), 261-281.

Anderson, R., \& Estle, G. (2001). Predicting level of mental health care among children served in a delivery system in a rural state. Journal Of Rural Health, 17(3), 259265. 
Anderson, R. L., Lyons, J. S., Giles, D. M., Price, J. A., \& Estle, G. (2003). Reliability of the Child and Adolescent Needs and Strengths-Mental Health (CANS-MH) scale. Journal Of Child \& Family Studies, 12(3), 279-289.

Armour, M. P., \& Schwab J. (2007). Characteristics of difficult-to-place youth in state custody: a profile of the exceptional care pilot project population. Child Welfare, 86(3), 71-96.

Baker, A., Schneiderman, M., \& Licandro, V. (2017). Mental health referrals and treatment in a sample of youth in foster care. Children \& Youth Services Review, $78,18-22$.

Baker-Ericzen, M. J., Jenkins, M. M., Park, S., \& Garland, A. F. (2015). Clinical decision-making in community children's mental health: Using innovative methods to compare clinicians with and without training in evidence-based treatment. Child \& Youth Care Forum, 44(1), 133-157.

Barcikowski, R. S. (1981). Statistical power with group mean as the unit of analysis. Journal of Educational Statistics, 6(3), 267-285.

Barlow, D. H. (2005). What's new about evidence-based assessment? Psychological Assessment, 17, 308-311.

Barwick, M. (2011). Master's level clinician competencies in child and youth behavioral healthcare. Emotional \& Behavioral Disorders in Youth, 11(2), 32-40.

Beach, L. R. (1993). Broadening the definition of decision making: The role of prechoice screening of options. Psychological Science (0956-7976), 4(4), 215-220. https://doi-org.echo.louisville.edu/10.1111/j.1467-9280.1993.tb00264.x

Beach, L. R. \& Lipshitz, R. (1993). Why classical decision theory is an inappropriate standard for evaluating and aiding most human decision making. In Klein, G. A., Orasanu, J., Calderwood, R., \& Zsambok, C. E. (Eds.). Decision making in action: Models and methods (pp. 21-35). Norwood, NJ: Ablex Publishing.

Beidas, R. S., Marcus, S., Aarons, G. A., Hoagwood, K. E., Schoenwald, S., Evans, A. C., \& ... Mandell, D. S. (2015). Predictors of community therapists' use of therapy techniques in a large public mental health system. JAMA Pediatrics, 169(4), 374382. doi:10.1001/jamapediatrics.2014.3736

Beidas, R. S., Stewart, R. E., Adams, D. R., Fernandez, T., Lustbader, S., Powell, B. J., \& ... Barg, F. K. (2016). A multi-level examination of stakeholder perspectives of implementation of evidence-based practices in a large urban publicly-funded mental health system. Administration And Policy In Mental Health And Mental Health Services Research, 43(6), 893-908. doi:10.1007/s10488-015-0705-2 
Bledsoe-Mansori, S. E., Bellamy, J. L., Wike, T., Grady, M., Dinata, E., Killian-Farrell, C., \& Rosenberg, K. (2013). Agency-university partnerships for evidence-based practice: A national survey of schools of social work. Social Work Research, 37(3), 179-193.

Bloom, S. (2013). The sanctuary model: A best-practices approach to organizational change. In V. L. Vandiver, V. L. Vandiver (Eds.), Best practices in community mental health: A pocket guide (pp. 303-313). Chicago, IL, US: Lyceum Books.

Bongers, I. L., Koot, H. M., van der Ende, J., \& Verhulst, F. C. (2003). The normative development of child and adolescent problem behavior. Journal of Abnormal Psychology, 112(2), 179-192.

Bordley, R. F. (2001). Naturalistic decision making and prescriptive decision theory. Journal of Behavioral Decision Making, 14(5), 355-357.

Briggs, E. C., Greeson, J. P., Layne, C. M., Fairbank, J. A., Knoverek, A. M., \& Pynoos, R. S. (2012). Trauma exposure, psychosocial functioning, and treatment needs of youth in residential care: Preliminary findings from the NCTSN core data set. Journal Of Child \& Adolescent Trauma, 5(1), 1-15. doi:10.1080/19361521.2012.646413

Burns, B., Phillips, S., Wagner, H., Barth, R., Kolko, D., Campbell, Y., \& Landsverk, J. (2004). Mental health need and access to mental health services by youths involved with child welfare: A national survey. Journal of the American Academy of Child and Adolescent Psychiatry, 43, 960-970.

Chang, X., Jiang, X., Mkandarwire, T., \& Shen, M. (2019). Associations between adverse childhood experiences and health outcomes in adults aged 18-59 years. PLoS ONE, 14(2), 1-11.

Cheng, T. C., \& Lo, C. C. (2011). A longitudinal analysis of some risk and protective factors in marijuana use by adolescents receiving child welfare services. Children and Youth Services Review, 33(9), 1667-1672.

Child and Family Services Reviews (2017). Family assessment. Retrieved from https://training.cfsrportal.acf.hhs.gov/section-2-understanding-child-welfaresystem/3026

Child Welfare League of America, I. D. (2008). Children's Voice. Volume 17, Number 3, May/June 2008. Child Welfare League of America.

Choi, S., \& Ryan, J. P. (2007). Co-occurring problems for substance abusing mothers in child welfare: Matching services to improve family reunification. Children \& Youth Services Review, 29(11), 1395-1410. 
Chor, K. B., McClelland, G. M., Weiner, D. A., Jordan, N., \& Lyons, J. S. (2012). Predicting outcomes of children in residential treatment: A comparison of a decision support algorithm and a multidisciplinary team decision model. Children and Youth Services Review, 34(2), 2345-2352.

Chor, K. B., McClelland, G. M., Weiner, D. A., Jordan, N., \& Lyons, J. S. (2013). Patterns of out-of-home placement decision-making in child welfare. Children and Youth Services Review, 37(10), 871-882

Cole, E. S. (1995). Becoming family centered: Child welfare's challenge. Families in Society, 76(3), 163-172.

Collin-Vezina, D., Coleman, K., Milne, L., Sell, J., \& Daigneault, I. (2011). Trauma experiences, maltreatment-related impairments, and resilience among child welfare youth in residential care. International Journal of Mental Health and Addiction, 9(5), 577-589.

Conradi, L., Wherry, J., \& Kisiel, C. (2011). Linking child welfare and mental health using trauma-informed screening and assessment practices. Child Welfare, 90(6), $129-147$.

Cook, J. M., Schnurr, P. P., Biyanova, T., \& Coyne, J. C. (2009). Apples don't fall far from the tree: Influences on psychotherapists' adoption and sustained use of new therapies. Psychiatric Services (Washington, D.C.), 60(5), 671-676. doi:10.1176/appi.ps.60.5.671

Copeland, W. E., Keeler, G., Angold, A., \& Costello, E. J. (2007). Traumatic events and posttraumatic stress in childhood. Archives Of General Psychiatry, 64(5), 577584.

Cordell, K. D., Snowden, L. R., \& Hosier, L. (2016). Patterns and priorities of service need identified through the Child and Adolescent Needs and Strengths (CANS) assessment. Children \& Youth Services Review, 60, 129-135. doi:10.1016/j.childyouth.2015.11.020

Craig, C. D. \& Sprang, G. (2010). Factors associated with the use of evidence-based practices to treat psychological trauma by psychotherapists with trauma treatment expertise. Journal of Evidence-Based Social Work, 7(5), 488-509

Creed, T., Wolk, C., Feinberg, B., Evans, A., \& Beck, A. (2016). Beyond the label: Relationship between community therapists' self-report of a cognitive behavioral therapy orientation and observed skills. Administration \& Policy in Mental Health \& Mental Health Services Research, 43(1), 36-43. 
Dickerson, J. F., Lynch, F. L., Leo, M. C., DeBar, L. L., Pearson, J., \& Clarke, G. N. (2018). Cost-effectiveness of cognitive behavioral therapy for depressed youth declining antidepressants. Pediatrics, 141(2), 1-9.

Dilley, J. B., Weiner, D. A., Lyons, J. S., \& Martinovich, Z. (2007). The validity of the Child and Adolescent Needs and Strengths assessment. Poster session presented at the convention of the American Psychological Association, Toronto, Ontario.

Dorsey, S., Burns, B., Southerland, D., Cox, J., Wagner, H., \& Farmer, E. (2012). Prior trauma exposure for youth in treatment foster care. Journal Of Child \& Family Studies, 21(5), 816-824. doi:10.1007/s10826-011-9542-4

Drisko, J. (2017). Active collaboration with clients: An underemphasized but vital part of evidence-based practice. Social Work, 62(2), 114-121.

Drisko, J. W. \& Grady, M. D. (2015). Evidence-based practice in social work: A contemporary perspective. Clinical Social Work Journal, 43(3), 274-282.

Dunleavy, A. M., \& Leon, S. C. (2011). Predictors for resolution of antisocial behavior among foster care youth receiving community-based services. Children \& Youth Services Review, 33(11), 2347-2354. doi:10.1016/j.childyouth.2011.08.005

Eells, T. D. \& Lombart, K. G. (2003). Case formulation and treatment concepts among novice, experienced, and expert cognitive-behavioral and psychodynamic therapists. Psychotherapy Research, 13(2), 187-204.

Fallot, R. D., \& Harris, M. (2002). The Trauma Recovery and Empowerment Model (TREM): Conceptual and practical issues in a group intervention for women. Community Mental Health Journal, 38(6), 475-485.

Falvey, J. E., Bray, T. E., \& Hebert, D. J. (2005). Case conceptualization and treatment planning: Investigation of problem-solving and clinical judgment. Journal of Mental Health Counseling, 27(4), 348-372.

Falzer, P. R. (2004). Cognitive schema and naturalistic decision making in evidencebased practices. Journal Of Biomedical Informatics, 37(2), 86-98.

Fettes, D. L., Aarons, G. A., \& Green, A. E. (2013). Higher rates of adolescent substance use in child welfare versus community populations in the United States. Journal of Studies on Alcohol and Drugs, 74(6), 825-834.

Garland, A. F., Kruse, M., \& Aarons, G. A. (2003). Clinicians and outcome measurement: What's the use? Journal of Behavioral Health Services \& Research, 30(4), 393-405. 
Gaudiano, B. A. (2008). Cognitive-behavioral therapies: Achievements and challenges. Evidence Based Mental Health, 11(1), 5-7.

Gauthier-Duchesne, A., Hébert, M., \& Daspe, M.-È. (2017). Gender as a predictor of posttraumatic stress symptoms and externalizing behavior problems in sexually abused children. Child Abuse \& Neglect, 64, 79-88.

Gerrity, E. \& Folcarelli, C. (2008). Child traumatic stress: What every policymaker should know. Durham, NC: National Center for Child Traumatic Stress.

Gibbs, L., \& Gambrill, E. (2002). Evidence-based practice: Counterarguments to objections. Research On Social Work Practice, 12(3), 452-476.

Gilgun, J. F. (2005). The four cornerstones of evidence-based practice in social work. Research on Social Work Practice, 15(1), 52-61.

Gioia, D. \& Dziadosz, G. (2008). Adoption of evidence-based practices in community mental health: A mixed-method study of practitioner experience. Community Mental Health Journal, 44(5), 347-357. doi:10.1007/s10597-008-9136-9

Godinet, M. T., Li, F., \& Berg, T. (2014). Early childhood maltreatment and trajectories of behavioral problems: Exploring gender and racial differences. Child Abuse \& Neglect, 38(3), 544-556

Goldstein, A., Wekerle, C., Tonmyr, L., Thornton, T., Waechter, R., Pereira, J., \& Chung, R. (2011). The relationship between post-traumatic stress symptoms and substance use among adolescents involved with child welfare: Implications for emerging adulthood. International Journal of Mental Health \& Addiction, 9(5), $507-524$.

Grabbe, L., \& Miller-Karas, E. (2018). The Trauma Resiliency Model: A "bottom-up" intervention for trauma psychotherapy. Journal Of The American Psychiatric Nurses Association, 24(1), 76-84.

Grady, M. D. \& Disko, J. W. (2014). Thorough clinical assessment: The hidden foundation of evidence-based practice. Families in Society: The Journal of Contemporary Social Services, 95(1), 5-14.

Grady, M. D. \& Keenan, E. K. (2014). Beyond the manual: Using research and evidence in social work practice. Clinical Social Work Journal, 42(1), 101-106.

Gray, M. J., Elhai, J. D., \& Schmidt, L. O. (2007). Trauma professionals' attitudes toward and utilization of evidence-based practices. Behavior Modification, 31(6), 732748. 
Greeson, J. P., Briggs, E. C., Kisiel, C. L., Layne, C. M., Ake, G. I., Ko, S. J., \& ... Fairbank, J. A. (2011). Complex trauma and mental health in children and adolescents placed in foster care: Findings from the National Child Traumatic Stress Network. Child Welfare, 90(6), 91-108.

Griffin G, Martinovich Z, Gawron T, Lyons J. S. (2009). Strengths moderate the impact of trauma on risk behaviors in child welfare. Residential Treatment for Children \& Youth, 26(2), 105-118.

Griffin, G., McClelland, G., Holzberg, M., Stolbach, B., Maj, N., \& Kisiel, C. (2011). Addressing the impact of trauma before diagnosing mental illness in child welfare. Child Welfare, 90(6), 69-89.

Groenier, M., Pieters, J. M., Witteman, C. L. M., Lehmann, S. R. S. (2014). The effect of client case complexity on clinical decision making. European Journal of Psychological Assessment, 30(2), 150-158.

Gutierrez, D., Fox, J., Jones, K., \& Fallon, E. (2018). The treatment planning of experienced counselors: A qualitative examination. Journal of Counseling \& Development, 96(1), 86-96.

Haby, M. M., Tonge, B., Littlefield, L., Carter, R., \& Vos, T. (2004). Cost-effectiveness of cognitive behavioural therapy and selective serotonin reuptake inhibitors for major depression in children and adolescents. Australian \& New Zealand Journal of Psychiatry, 38(8), 579-591.

Hastie, R. \& Dawes, R. M. (2010). Rational choice in an uncertain world: The psychology of judgment and decision making ( $2^{\text {nd }}$ ed.). Los Angeles, CA: SAGE

Haynes, R., Devereaux, P., \& Guyatt, G. (2002). Clinical expertise in the era of evidencebased medicine and patient choice (editorial). Vox Sanguinis, 83(S1), 383-386.

Heck, R. H., Thomas, S. L., \& Tabata, L. N. (2012). Multilevel modeling of categorical outcomes using IBM SPSS. New York, NY: Routledge.

Hoffmann, J. P., \& Cerbone, F. G. (2002). Parental substance use disorder and the risk of adolescent drug abuse: An event history analysis. Drug and Alcohol Dependence, $66(3), 255-264$.

Hofmann, S., Asnaani, A., Vonk, I., Sawyer, A., \& Fang, A. (2012). The efficacy of cognitive behavioral therapy: A review of meta-analyses. Cognitive Therapy \& Research, 36(5), 427-440. https://doi-org.echo.louisville.edu/10.1007/s10608012-9476-1

Hoge, R. D. (2002). Standardized Instruments for assessing risk and need in youthful offenders. Criminal Justice \& Behavior, 29(4), 380-396. 
Holmes, M. R., Yoon, S., Voith, L. A., Kobulsky, J. M., \& Steigerwald, S. (2015). Resilience in physically abused children: Protective factors for aggression. Behavioral Sciences (2076-328X), 5(2), 176-189. https://doiorg.echo.louisville.edu/10.3390/bs5020176

Hox, J. J. (1995). Applied multilevel analysis. Amsterdam: TT-Publikaties.

Hox, J. J., Moerbeek, M., \& van de Schhot, R. (2018). Multilevel analysis: Techniques and applications $\left(3^{\text {rd }}\right.$ ed.). New York, NY: Routledge.

Igelman, R., Taylor, N., Gilbert, A., Ryan, B., Steinberg, A., Wilson, C., \& Mann, G. (2007). Creating more trauma-informed services for children using assessmentfocused tools. Child Welfare, 86(5), 15-33.

Ingram, S. D., Cash, S. J., Oats, R. G., Simpson, A., \& Thompson, R. W. (2015). Development of an evidence-informed in-home family services model for families and children at risk of abuse and neglect. Child \& Family Social Work, 20(2), 139-148.

Jensen-Doss, A. (2011). Practice involves more than treatment: How can evidence-based assessment catch up to evidence-based treatment? Clinical Psychology: Science \& Practice, 18(2), 173-177. https://doi-org.echo.louisville.edu/10.1111/j.14682850.2011.01248.x

Kentucky Cabinet for Health \& Family Services, Department for Community Based Services (2018). Statewide foster care fact sheet. Retrieved from http://chfs.ky.gov/dcbs /diqi/datafactsheets.htm

Kilmer, R. P., Cook, J. R., \& Munsell, E. P. (2010). Moving from principles to practice: Recommended policy changes to promote family-centered care. American Journal of Community Psychology, 46(3-4), 332-341.

Kisiel, C., Fehrenbach, T., Small, L., \& Lyons, J. S. (2009). Assessment of complex trauma exposure, responses, and service needs among children and adolescents in child welfare. Journal of Child \& Adolescent Trauma, 2(1), 143-160.

Kisiel, C., Conradi, L., Fehrenbach, T., Torgersen, E., \& Briggs, E. C. (2014). Assessing the effects of trauma in children and adolescents in practice settings. Child And Adolescent Psychiatric Clinics Of North America, 23(2), 223-242. doi:10.1016/j.chc.2013.12.007

Kisiel, C., Fehrenbach, T., Torgersen, E., Stolbach, B., McClelland, G., Griffin, G., \& Burkman, K. (2014). Constellations of interpersonal trauma and symptoms in child welfare: Implications for a developmental trauma framework. Journal of Family Violence, 29(1), 1-14. doi:10.1007/s10896-013-9559-0 
Kisiel, C., Summersett-Ringgold, F., Weil, L., \& McClelland, G. (2017). Understanding strengths in relation to complex trauma and mental health symptoms within child welfare. Journal Of Child \& Family Studies, 26(2), 437-451. doi:10.1007/s10826016-0569-4

Klein, G. A. (1998). Sources of power: How people make decisions. Cambridge, MA: MIT Press.

Klein, G. A. (2008). Naturalistic decision making. Human Factors, 50(3), pp. 456-460. doi:10.1518/001872008X88385

Klein, G. A., Calderwood, R. \& Clinton-Cirocco, A. (1986). Rapid decision making on the fireground. Proceedings of the Human Factors and Ergonomics Society $30^{\text {th }}$ Annual Meeting, 1, 576-580.

Ko, S. J., Ford, J. D., Kassam-Adams, N., Berkowitz, S. J., Wilson, C., Wong, M., \& ... Layne, C. M. (2008). Creating trauma-informed systems: Child welfare, education, first responders, health care, juvenile justice. Professional Psychology: Research \& Practice, 39(4), 396-404. doi:10.1037/0735-7028.39.4.396

Kobulsky, J. M. (2017). Gender differences in pathways from physical and sexual abuse to early substance use. Children \& Youth Services Review, 83, 25-32.

Kolko, D. J., Herschell, A. D., Costello, A. H., \& Kolko, R. P. (2009). Child welfare recommendations to improve mental health services for children who have experienced abuse and neglect: A national perspective. Administration \& Policy in Mental Health \& Mental Health Services Research, 36(1), 50-62.

Kreft, I. \& DeLeeuw, J. (1998). Introducing multilevel modeling. Thousand Oaks, CA: SAGE.

Kwaadsteniet, L. d., Hagmayer, Y., Krol, N. M., \& Witteman, C. M. (2010). Causal client models in selecting effective interventions: A cognitive mapping study. Psychological Assessment, 22(3), 581-592.

Lang, J. M., Randall, K. G., Delaney, M., \& Vanderploeg, J. J. (2017). A model for sustaining evidence-based practices in a statewide system. Families In Society, 98(1), 18-26.

Lardner, M. D. (2015). Are restrictiveness of care decisions based on youth level of need? A multilevel model analysis of placement levels using the Child and Adolescent Needs and Strengths assessment. Residential Treatment For Children \& Youth, 32(3), 195-207. doi:10.1080/0886571X.2015.1080993

Lehmann, S., Havik, O. E., Havik, T., \& Heiervang, E. R. (2013). Mental disorders in foster children: a study of prevalence, comorbidity and risk factors. Child \& 
Adolescent Psychiatry \& Mental Health, 7(1), 1-23. https://doiorg.echo.louisville.edu/10.1186/1753-2000-7-39

Lipshitz, R. (1994). Decision making in three modes. Journal for the Theory of Social Behavior, 24(1), 47-65.

Lipshitz, R., Klein, G., Orasanu, J., \& Salas, E. (2001). Taking stock of naturalistic decision making. Journal of Behavioral Decision Making, 14(5), 331-352.

Lee, J. S., \& Logan-Greene, P. (2017). Patterns of family service needs and caregiverchild relationships among families at risk of child welfare involvement. Child \& Family Social Work, 22(4), 1515-1524.

Lucock, M. P., Hall, P., \& Noble, R. (2006). A survey of influences on the practice of psychotherapists and clinical psychologists in training in the UK. Clinical Psychology and Psychotherapy, 13(1), 123-130.

Lushin, V., Becker-Haimes, E. M., Mandell, D., Conrad, J., Kaploun, V., Bailey, S., ... Beidas, R. S. (2019). What motivates mental health clinicians-in-training to implement evidence-based assessment? A survey of social work trainees. Administration And Policy In Mental Health. https://doiorg.echo.louisville.edu/10.1007/s10488-019-00923-4

Lyons, J. S. (2009). Communimetric: A communication theory of measurement in human service settings. New York, NY: Springer.

Macdonald, G., Higgins, J. P. T., Ramchandani, P., Valentine, J. C., Bronger, L. P., Klein, P., ... Taylor, M. (2012). Cognitive-behavioural interventions for children who have been sexually abused. The Cochrane Database Of Systematic Reviews, (5), CD001930.

Marenko, M., Newby, M., Lee, J., Courtney, M. \& Brennan, K. (2009). Evaluation of Washington's solution-based casework practice model: Part IV-Baseline parent survey. Seattle, WA: Partners for Our Children

Mash, E. J., \& Hunsley, J. (2005). Evidence-based assessment of child and adolescent disorders: Issues and challenges. Journal Of Clinical Child \& Adolescent Psychology, 34(3), 362-379.

McBeath, B., Briggs, H. E., Aisenberg, E. (2010). Examining the premises supporting the empirically supported intervention approach to social work practice. Social Work, 55(4), 347-357.

McCrae JS. (2009). Emotional and behavioral problems reported in child welfare over 3 years. Journal of Emotional \& Behavioral Disorders, 17(1), 17-28. 
McKelvey, R. \& Zavoina, W. (1975). A statistical model for the analysis of ordinal level dependent variables. Journal of Mathematical Sociology, 4, 103-120.

Meyers, L. S., Gamst, G., \& Guarino, A. J. (2013). Applied multivariate research: Design and implementation ( $2^{\text {nd }}$ ed.). Los Angeles, CA:SAGE

Miller, J. \& Maloney, C. (2013). Practitioner compliance with risk/needs assessment tools: A theoretical and empirical assessment. Criminal Justice and Behavior, 40(7), 716-736.

Milne, L. \& Collin-Vezina, D. (2014). Assessment of children and youth in child protective services out-of-home care: An overview of trauma measures. Psychology of Violence, 5(2), 122-132.

Moineddin, R., Matheson, F. I., \& Glazier, R. H. (2007). A simulation study of sample size for multilevel logistic regression models. BMC Medical Research Methodology, 7(1), 34.

Mullen, E. J., Bledsoe, S. E., \& Bellamy, J. L. (2008). Implementing evidence-based social work practice. Research On Social Work Practice, 18(4), 325-338. doi:10.1177/1049731506297827

Navalta, C. P., McGee, L., \& Underwood, J. (2018). Adverse childhood experiences, brain development, and mental health: A call for neurocounseling. Journal of Mental Health Counseling, 40(3), 266. https://doiorg.echo.louisville.edu/10.17744/mehc.40.3.07

Nelson, T. D., \& Steele, R. G. (2007). Predictors of Practitioner Self-reported Use of Evidence-Based Practices: Practitioner Training, Clinical Setting, and Attitudes Toward Research. Administration \& Policy In Mental Health \& Mental Health Services Research, 34(3), 319-330. doi:10.1007/s10488-006-0111-x

Nelson, T. D., \& Steele, R. G. (2008). Influences on practitioner treatment selection: Best research evidence and other considerations. Journal Of Behavioral Health Services \& Research, 35(2), 170-178. doi:10.1007/s11414-007-9089-8

Nelson, T. D., Steele, R. G., \& Mize, J. A. (2006). Practitioner attitudes toward evidencebased practice: themes and challenges. Administration And Policy In Mental Health, 33(3), 398-409.

O'Hare, D. (1992). The "artful" decision maker: A framework model for aeronautical decision making. International Journal of Aviation Psychology, 2(3), 175. https://doi-org.echo.louisville.edu/10.1207/s15327108ijap0203_2 
Orasanu, J. \& Connolly, T. (1993). The reinvention of decision making. In Klein, G. A., Orasanu, J., Calderwood, R., \& Zsambok, C. E. (Eds.). Decision making in action: Models and methods (pp. 3-20). Norwood, NJ: Ablex Publishing.

Oswald, S. H., Heil, K., \& Goldbeck, L. (2010). History of maltreatment and mental health problems in foster children: A review of the literature. Journal of Pediatric Psychology, 35(5), 462-472.

Özabac1, N. (2011). Cognitive behavioural therapy for violent behaviour in children and adolescents: A meta-analysis. Children \& Youth Services Review, 33(10), 19891993.

Parker, E. M., Jacobson, J., Pullman, M. D., \& Kerns, S. E. U. (2019). Identifying psychosocial problems among children and youth in the child welfare system using the PSC-17: Exploring convergent and discriminant validity with multiple informants. Child Psychiatry \& Human Development, 50(1), 108-120.

Pittenger, S. L., Moore, K. E., Dworkin, E. R., Crusto, C. A., \& Connell, C. M. (2018). Risk and protective factors for alcohol, marijuana, and cocaine use among child welfare-involved youth. Children and Youth Services Review, 95(1), 88-94.

Pignotti, M. \& Thyer, B. A. (2009). Use of novel unsupported and empirically supported therapies by licensed clinical social workers: An exploratory study. Social Work Research, 33(1), 5-16.

Ponniah, K., Weissman, M. M., Bledsoe, S. E., Verdeli, H., Gameroff, M. J., Mufson, L., ... Wickramaratne, P. (2011). Training in structured diagnostic assessment using the DSM-IV criteria. Research on Social Work Practice, 21(4), 452-457.

Powers, D. A. (2012). Multilevel models for binary data. New Directions for Institutional Research, 154, 57-75.

Praed Foundation. (2016). Child and Adolescent Needs and Strengths: 2016 reference guide. Chicago, IL: Chapin Hall

Praed Foundation. (2015). Child and Adolescent Needs and Strengths (CANS). Retrieved from https://praedfoundation.org/tools/the-child-and-adolescent-needs-andstrengths-cans/

Pruitt, J. S., Cannon-Bowers, J. A., \& Salas, E. (1997). In search of naturalistic decisions. In R. Flin, E. Salas, M. Strub, \& L. Martin (Eds.), Decision making under stress: Emerging themes and applications (pp. 29-42). Aldershot, UK: Ashgate.

Rasmussen, B. (2018). A critical examination of CBT in clinical social work practice. Clinical Social Work Journal, 46(3), 165-173. https://doiorg.echo.louisville.edu/10.1007/s10615-017-0632-7 
Regehr, C., Stern, S., \& Shlonsky, A. (2007). Operationalizing evidence-based practice: The development of an institute for evidence-based social work. Research On Social Work Practice, 17(3), 408-416.

Roberts, A. R. \& Yeager, K. R. (2004). Evidence -based practice manual: Research and outcome measures in health and human services. New York, NY: Oxford University Press.

Robson, K. \& Pevalin, D. (2016). Multilevel modeling in plain language. Los Angeles, CA: SAGE.

Romanelli, L. H., Landsverk, J., Levitt, J. M., Leslie, L. K., Hurley, M. M., Bellonci, C., \& ... Jensen, P. S. (2009). Best practices for mental health in child welfare: Screening, assessment, and treatment guidelines. Child Welfare, 88(1), 163-188.

Rosanbalm, K. K., Snyder, E. H., Lawrence, C. N., Coleman, K., Frey, J. J., van den Ende, J. B., \& Dodge, K. A. (2016). Child wellbeing assessment in child welfare: A review of four measures. Children \& Youth Services Review, 68, 1-16.

Rubin, A. (2008). Practitioner's guide to using research for evidence-based practice. Hoboken, NJ: Wiley.

Rubin, A., \& Parrish, D. (2007). Views of evidence-based practice among faculty in master of social work programs: a national survey. Research On Social Work Practice, 17(1), 110-122.

Sackett, D. L., Richardson, W. S., Rosenberg, W., \& Haynes, R. B. (1997). Evidencebased medicine: How to practice \& teach EBM. New York: Churchill Livingstone.

Salazar, A. M., Keller, T. E., Gowen, L. K., \& Courtney, M. E. (2013). Trauma exposure and PTSD among older adolescents in foster care. Social Psychiatry \& Psychiatric Epidemiology, 48(4), 545-551.

Saunders, B. E. (2015). Expanding evidence-based practice to service planning in child welfare. Child Maltreatment, 20(1), 20-22. doi:10.1177/1077559514566299

Scheidt, C. E., Burger, T., Strukely, S., Hartmann, A., Fritzsche, K. \& Wirsching, M. (2003). Treatment selection in private practice psychodynamic psychotherapy: A naturalistic prospective longitudinal study. Psychotherapy Research, 13(3), 293305.

Schoeneberger, J. A. (2016). The impact of sample size and other factors when estimating multilevel logistic models. The Journal of Experimental Education, 84(2), 373-397. 
Schoenwald, S. K., \& Hoagwood, K. (2001). Effectiveness, transportability, and dissemination of interventions: what matters when?. Psychiatric Services (Washington, D.C.), 52(9), 1190-1197.

Schottenbauer, M. A., Glass, C. R., \& Arnkoff, D. B. (2007). Decision making and psychotherapy integration: Theoretical considerations, preliminary data, and implications for future research. Journal of Psychotherapy Integration, 17(3), 225-250.

Schwalbe, C. (2004). Re-visioning risk assessment for human service decision making. Children \& Youth Services Review, 26(6), 561-576.

Sexton, T. L. \& Kelley, S. D. (2010). Finding the common core: Evidence-based practices, clinically relevant evidence, and core mechanisms of change. Administration \& Policy In Mental Health \& Mental Health Services Research, 37(1/2), 81-88. doi:10.1007/s10488-010-0277-0

Shadish, W. R., Cook, T. D., \& Campbell, D. T. (2002). Experimental and quasiexperimental designs for generalized causal inference. Belmont, CA: Wadsworth Cengage Learning

Shlonsky, A., \& Gibbs, L. (2004). Will the real evidence-based practice please stand up? Teaching the process of evidence-based practice to the helping professions. Brief Treatment \& Crisis Intervention, 4(2), 137-153. doi:10.1093/brieftreatment/mhh011

Simmons, B. M. (2011). The complexity of evidence-based practice: A case study. Smith College Studies in Social Work, 81(2), 252-267.

Smithgall, C., Jarpe-Ratner, E., Gnedko-Berry, N., \& Mason, S. (2015). Developing and testing a framework for evaluating the quality of comprehensive family assessment in child welfare. Child Abuse \& Neglect, 44, 194-206.

Sommet, N. \& Morselli, D. (2017). Keep calm and learn multilevel logistic modeling: A simplified three-step procedure using Strata, R, Mplus, and SPSS. International Review of Social Psychology, 30(1), 203-218.

Sonu, S., Post, S., \& Feinglass, J. (2019). Adverse childhood experiences and the onset of chronic disease in young adulthood. Preventive Medicine, 123, 163-170. https://doi-org.echo.louisville.edu/10.1016/j.ypmed.2019.03.032

Spring, B. (2008). Health decision making: Lynchpin of evidence-based practice. Medical Decision Making: An International Journal Of The Society For Medical Decision Making, 28(6), 866-874. https://doiorg.echo.louisville.edu/10.1177/0272989X08326146 
Stadnick, N. A., Lau, A. S., Barnett, M., Regan, J., Aarons, G. A., \& Brookman-Frazee, L. (2017). Comparing agency leader and therapist perspectives on evidence-based practices: Associations with individual and organizational factors in a mental health system-driven implementation effort. Administration And Policy In Mental Health, doi:10.1007/s10488-017-0835-9

Stahmer, A. C., Leslie, L. K., Hurlburt, M., Barth, R. P., Webb, M. B., Landsverk, J., \& Zhang, J. (2005). Developmental and behavioral needs and service use for young children in child welfare. Pediatrics, 116(4), 891-900. https://doiorg.echo.louisville.edu/10.1542/peds.2004-2135

Tabachnick, B. G. \& Fidell, L. G. (2013). Using multivariate statistics (6 ${ }^{\text {th }}$ ed.). Boston, MA: Pearson.

Teater, B. (2017). Social work research and its relevance to practice: "The gap between research and practice continues to be wide." Journal of Social Service Research, 43(5), 547-565.

Thompson, H. M., \& Colvin, M. L. (2018). Perceived needs of therapeutic service providers in their work with families in the child welfare system. Child \& Adolescent Social Work Journal, 35(1), 57-71.

Thyer, B. A. (2004). What is evidence-based practice? Brief Treatment and Crisis Intervention, 4(2), 167-176

Thyer, B., Babcock, P., \& Tutweiler, M. (2017). Locating research-supported interventions for child welfare practice. Child \& Adolescent Social Work Journal, 34(2), 85-94. doi:10.1007/s10560-016-0478-9

Thyer, B. A., \& Myers, L. L. (2011). The quest for evidence-based practice: A view from the United States. Journal Of Social Work, 11(1), 8-25. doi: $10.1177 / 1468017310381812$

Thyer, B. B. \& Pignotti, M. (2011). Evidence-based practices do not exist. Clinical Social Work Journal, 39(4), 328-333.

Traube, D. E., James, S., Zhang, J., \& Landsverk, J. (2012). A national study of risk and protective factors for substance use among youth in the child welfare system. Addictive Behaviors, 37(5), 641-650.

Tremblay, R. E., Nagin, D. S., Séguin, J. R., Zoccolillo, M., Zelazo, P. D., Boivin, M., ... Japel, C. (2004). Physical aggression during early childhood: Trajectories and predictors. Pediatrics, 114(1), e43-e50. 
Tuten, M., Morris-Compton, D., Abrefa-Gyan, T., Hwang, J., \& Harrington, D. (2016). Predictors of the use of evidence-based interventions among National Association of Social Work (NASW) members. Journal Of Evidence-Informed Social Work, 13(3), 253-262. doi:10.1080/23761407.2015.1047109

U.S. Department of Health \& Human Services, Administration for Children and Families, Administration on Children, Youth and Families, Children's Bureau. (2017a). Child maltreatment 2015. Retrieved from http://www.acf.hhs.gov/programs/cb/research-data-technology/statisticsresearch/child-maltreatment

U.S. Department of Health \& Human Services, Administration for Children and Families, Administration on Children, Youth and Families, Children's Bureau. (2017b). Child welfare outcomes 2010-2014: Report to congress. Retrieved from https://www.acf.hhs.gov/cb/resource/cwo-10-14

Vaughn, M. G., Ollie, M. T., McMillen, J. C., Scott, L., \& Munson, M. (2007). Substance use and abuse among older youth in foster care. Addictive Behaviors, 32(9), 1929-1935.

van de Luitgaarden, G. M. J. (2009). Evidence-based practice in social work: Lessons from judgment and decision-making theory. British Journal of Social Work, 39(2), 243-260. https://doi-org.echo.louisville.edu/10.1093/bjsw/bcm117

van Steensel, F. J. A., Dirksen, C. D., \& Bögels, S. M. (2014). Cost-effectiveness of cognitive-behavioral therapy versus treatment as usual for anxiety disorders in children with autism spectrum disorder. Research in Autism Spectrum Disorders, 8(2), 127-137.

Walker, J. S., Briggs, H. E., Koroloff, N., \& Frieson, B. J. (2007). Implementing and sustaining evidence-based practice in social work. Journal of Social Work Education, 43(3), 361-375.

Wamser-Nanney, R., \& Cherry, K. E. (2018). Children's trauma-related symptoms following complex trauma exposure: Evidence of gender differences. Child Abuse \& Neglect, 77, 188-197.

Webb, S. A. (2001). Some considerations on the validity of evidence-based practice in social work. British Journal of Social Work, 31(1), 57-79. https://doiorg.echo.louisville.edu/10.1093/bjsw/31.1.57

Weiner, D., Leon, S., \& Stiehl, M. (2011). Demographic, clinical, and geographic predictors of placement disruption among foster care youth receiving wraparound services. Journal of Child \& Family Studies, 20(6), 758-770. 
Weissman, M. M., Verdeli, H., Gameroff, M. J., Bledsoe, S. E., Betts, K., Mufson, L., ... Wickramaratne, P. (2006). National survey of psychotherapy training in psychiatry, psychology, and social work. Archives Of General Psychiatry, 63(8), 925-934.

White, S. \& Stancombe, J. (2003). Clinical judgment in the health and welfare professions: Extending the evidence base. Philadelphia, PA: Open University Press

Willging, C. E., Gunderson, L., Green, A. E., Jaramillo, E. T., Garrison, L., Ehrhart, M. G., \& Aarons, G. A. (2018). Perspectives from community-based organizational managers on implementing and sustaining evidence-based interventions in child welfare. Human Service Organizations: Management, Leadership \& Governance, 42(4), 359-379.

Witteman, C., \& Kunst, H. (1997). Planning the treatment of a depressed patient. Clinical Psychology \& Psychotherapy, 4(3), 157-171.

Witteman, C., \& Koele, P. (1999). Explaining treatment decisions. Psychotherapy Research, 9(1), 100-114. doi:10.1093/ptr/9.1.100.

Wright, D. B. (1997). Understanding statistics: An introduction for the social sciences. Thousand Oaks, CA: Sage.

Yampolskaya, S., Armstrong, M. I., \& Vargo, A. C. (2007). Factors associated with exiting and reentry into out-of-home care under community-based care in Florida. Children \& Youth Services Review, 29(10), 1352-1367.

Yampolskaya, S., Chuang, E., \& Walker, C. (2019). Trajectories of substance use among child welfare-involved youth: Longitudinal associations with child maltreatment history and emotional behavioral problems. Substance Use \& Misuse, 54(3), 437448.

Yoon, S., Tebben, E., \& Lee, G. (2017). Early childhood aggression among child welfare involved children: The interplay between the type of child maltreatment and ecological protective factors. Children \& Youth Services Review, 81, 72-80. https://doi-org.echo.louisville.edu/10.1016/j.childyouth.2017.07.030

Young, J. \& Beck, A. T. (1980). Cognitive therapy scale rating manual. Bala Cynwyd, PA: Beck Institute for Cognitive Behavior Therapy.

Zsambok, C. E. (1997). Naturalistic decision making: Where are we now? In C. E. Zsambok \& G. A. Klein (Eds.). Naturalistic decision making (pp. 3-16). Mahwah, NJ: Erlbaum 
Zuber, I. (2000). Patient's own problem formulations and recommendations for psychotherapy. Journal of Psychotherapy Integration, 10(4), 403-414 


\section{CURRICULUM VITA}

NAME:

ADDRESS:

DOB:

EDUCATION:

PUBLICATIONS:

\section{PRESENTATIONS:}

BOOKS:
Alton Nathaniel Verbist

4528 South $2^{\text {nd }}$ Street

Louisville, KY 40214

Clarksville, Tennessee - December 6, 1979

B.A., Political Science, Philosophy, and Economics Union University

$1998-2003$

M.S.S.W, Social Work

University of Louisville

2012 - 2014

Ph.D. (in progress), Social Work

University of Louisville

2014 - Present

Sears, J. S., Verbist, A. N., Wilfong, J., Thomas, T. A., Mount, S., Sabree, R., \& van Zyl, M. A. (2018). Mapping the science of social work debate: An exercise in doctoral student education. Journal of Social Work Education, 54(3), 598-606.

Collins-Camargo, C., Shlonsky, A., \& Verbist, A. N. (2017). Introduction to the Special Issue on Child Welfare Literature Reviews: Literature Reviews, Systematic Reviews and MetaAnalyses-How Can Child Welfare Administrators, Practitioners and Researchers Determine What We Know? Journal of Public Child Welfare, 11(4/5), 379.

Verbist, A. N. (2016, November 4). Trauma exposure among youth in therapeutic foster care and residential treatment. $62^{\text {nd }}$ Annual Program Meeting - Council on Social Work Education

Lawson, T., Verbist, A. N., Peterson, S., \& Galle, J. (2014, November 12). Panel discussion - From soldier to civilian: Transitioning veterans' experiences. Employment \& Transitions Over the Lifespan: Promoting Veteran Cultural Competence.

Lawson, T., Faul, A. \& Verbist, A. N. (2019). Research and statistics for social workers. New York, NY: Routledge. 